\title{
SPECIAL VALUES OF ANTICYCLOTOMIC L-FUNCTIONS FOR MODULAR FORMS
}

\author{
MASATAKA CHIDA AND MING-LUN HSIEH
}

\begin{abstract}
In this article, we generalize some works of Bertolini-Darmon and Vatsal on anticyclotomic $L$ functions attached to modular forms of weight two to higher weight case. We construct a class of anticyclotomic $p$-adic $L$-functions for ordinary modular forms and derive the functional equation and the interpolation formula at all critical specializations. Moreover, we prove results on the vanishing of $\mu$-invariant of these $p$-adic $L$ functions and the non-vanishing of central $L$-values with anticyclotomic twists.
\end{abstract}

\section{Contents}

Introduction

1. Notation and definitions

2. Gross points and modular forms on definite quaternion algebras 6

3. Special value formula 8

4. Theta elements and $p$-adic $L$-functions 18

5. The non-vanishing of theta elements modulo $\ell \quad 23$

6. The comparison between periods $\quad 28$

References

\section{INTRODUCTION}

The purpose of this article is to generalize works of Bertolini, Darmon and Vatsal on anticyclotomic $p$-adic $L$ functions attached to modular forms of weight two to higher weight. We construct anticyclotomic $p$-adic $L$ functions for ordinary modular forms and prove the interpolation property at all critical specializations and the functional equation. In addition, following the ideas of Vatsal, we prove results on the vanishing of the $\mu$-invariant of this anticyclotomic $p$-adic $L$-functions and the non-vanishing modulo $\ell$ of central $L$-values with anticyclotomic twists. To state our results precisely, we introduce some notation. Let $f \in S_{k}\left(\Gamma_{0}(N)\right)$ be an elliptic new form of weight $k$ (even) and conductor $N$. Let $p$ be a rational prime. Let $K$ be an imaginary quadratic field of discriminant $-D_{K}<0$. Then $K$ determines a factorization

$$
N=p^{n_{p}} N^{+} N^{-} \quad\left(p, N^{+} N^{-}\right)=1,
$$

where $N^{+}$(resp. $N^{-}$) is only divisible by primes that are split (resp. inert or ramified) in $K$. We assume that

$N^{-}$is the square-free product of an odd number of primes.

Fix a decomposition $N^{+} \mathcal{O}_{K}=\mathfrak{N}^{+} \overline{\mathfrak{N}^{+}}$. For each finite prime, let $\epsilon_{q}(f) \in\{ \pm 1\}$ denote the local root number of $f$, so $\epsilon_{q}(f)=1$ if $q \nmid N$, and $\epsilon_{q}(f)$ is the eigenvalue of Atkin-Lehner involution at $q$ if $q \mid N$. The global root number $\epsilon(f):=(-1)^{\frac{k}{2}} \prod_{q} \epsilon_{q}(f)$. Let

$$
f(q)=\sum_{n=1}^{\infty} \mathbf{c}_{n}(f) q^{n}
$$

be the $q$-expansion of $f$ at the infinity cusp. It is known that $\epsilon_{q}(f)=-q^{\frac{2-k}{2}} \mathbf{c}_{q}(f)$ if $q \| N$. Let $A_{p}$ be a complex root of $X^{2}-\mathbf{c}_{p}(f) X+p^{k-1}$ if $p \nmid N$ (or $X^{2}-\mathbf{c}_{p}(f) X$ if $\left.p \mid N\right)$. Let $K_{\infty}^{-}$be the anticyclotomic

Date: June 27, 2018.

2010 Mathematics Subject Classification. 11F67 11G15.

The first author is partly supported by Grant-in-Aid for Young Scientists (B) No. 23740015 and Hakubi project of Kyoto University. The second author is partly supported by National Science Council grant 101-2115-M-002-010-MY2. 
$\mathbf{Z}_{p}$-extension over $K$ and let $\Gamma^{-}=\operatorname{Gal}\left(K_{\infty}^{-} / K\right)$ be the Galois group. Denote by $\operatorname{rec}_{K}: \mathbf{A}_{K}^{\times} \rightarrow G_{K}^{a b}$ the geometrically normalized reciprocity law map. Fix embeddings $\iota_{\infty}: \overline{\mathbf{Q}} \hookrightarrow \mathbf{C}$ and $\iota_{p}: \overline{\mathbf{Q}} \hookrightarrow \mathbf{C}_{p}$. To each locally algebraic $p$-adic character $\hat{\chi}: \Gamma^{-} \rightarrow \mathbf{C}_{p}^{\times}$of ( $p$-adic) weight $(m,-m) \in \mathbf{Z}^{2}$, we can associate a Hecke character $\chi: \mathbf{A}_{K}^{\times} / K^{\times} \rightarrow \mathbf{C}^{\times}$of (archimedean) weight $(m,-m)$ defined by

$$
\chi(a):=\iota_{\infty} \iota_{p}^{-1}\left(\widehat{\chi}\left(\operatorname{rec}_{K}(a)\right)\left(\bar{a}_{p} / a_{p}\right)^{m}\right)\left(a_{\infty} / \bar{a}_{\infty}\right)^{m},
$$

where $a_{p} \in\left(K \otimes_{\mathbf{Q}} \mathbf{Q}_{p}\right)^{\times}$and $a_{\infty} \in\left(K \otimes_{\mathbf{Q}} \mathbf{R}\right)^{\times}$are the $p$-component and $\infty$-component of $a$. We call $\hat{\chi}$ the $p$-adic avatar of $\chi$. Let $\mathfrak{X}_{p}^{\text {crit }}$ be the set of critical specializations consisting of locally algebraic $p$-adic characters $\widehat{\chi}: \Gamma^{-} \rightarrow \mathbf{C}_{p}^{\times}$of weight $(m,-m)$ with

$$
-k / 2<m<k / 2 .
$$

Let $\mathcal{O}_{f}$ be the ring of integers of the Hecke field of $f$. Fix a prime $\lambda$ of $\overline{\mathbf{Q}}$ and let $\mathcal{O}_{f, \lambda}$ be the completion of $\mathcal{O}_{f}$ with respect to $\lambda$. Suppose that $\lambda$ is induced by $\iota_{p}$ (so $\lambda$ has residue characteristic $p$ ). Denote by $L(f / K, \chi, s)$ the Rankin $L$-series associated with $f$ and the theta series attached to $\chi$. Our first theorem is the construction of the anticyclotomic $p$-adic $L$-function attached to $f$ over $K$ with the explicit evaluation formula at critical specializations.

Theorem A. Suppose that

(a) $p>k-2$,

(b) $A_{p}$ is a $\lambda$-adic unit.

Then there exist an element $\Theta_{\infty} \in \mathcal{O}_{f, \lambda} \llbracket \Gamma^{-} \rrbracket$ and a complex number $\Omega_{f, N^{-}} \in \mathbf{C}^{\times}$such that for every $\hat{\chi} \in \mathfrak{X}_{p}^{\text {crit }}$ of weight $(m,-m)$ and conductor $p^{n}$, we have the following interpolation formula:

$$
\begin{aligned}
\widehat{\chi}\left(\Theta_{\infty}^{2}\right)= & \Gamma(k / 2+m) \Gamma(k / 2-m) \cdot \frac{L(f / K, \chi, k / 2)}{\Omega_{f, N^{-}}} \cdot e_{p}(f, \chi)^{2-\operatorname{ord}_{p}(N)} \cdot p^{n} A_{p}^{-2 n}\left(p^{n} D_{K}\right)^{k-2} \\
& \times u_{K}^{2} \sqrt{D_{K}} \cdot \epsilon_{p}(f)(-1)^{m} \prod_{q \mid\left(D_{K}, N^{-}\right)}\left(1-\epsilon_{q}(f)\right) \cdot \chi\left(\mathfrak{N}^{+}\right),
\end{aligned}
$$

where $u_{K}=\sharp\left(\mathcal{O}_{K}^{\times}\right) / 2$ and $e_{p}(f, \chi)$ is the $p$-adic multiplier given by

$$
e_{p}(f, \chi)= \begin{cases}1 & \text { if } n>0, \\ \left(1-\chi(\mathfrak{p}) p^{\frac{k-2}{2}} A_{p}^{-1}\right)\left(1-\chi(\overline{\mathfrak{p}}) p^{\frac{k-2}{2}} A_{p}^{-1}\right) & \text { if } n=0 \text { and } p=\mathfrak{p} \overline{\mathfrak{p}} \text { is split, } \\ 1-p^{k-2} A_{p}^{-2} & \text { if } n=0 \text { and } p=\mathfrak{p} \text { is inert, } \\ 1-\chi(\mathfrak{p}) p^{\frac{k-2}{2}} A_{p}^{-1} & \text { if } n=0 \text { and } p=\mathfrak{p}^{2} \text { is ramified. }\end{cases}
$$

Remark. (1) The existence of $A_{p}$ satisfying the assumption (b) is usually referred to the $p$-ordinary hypothesis for $f$, i.e. the $p$-th Fourier coefficient $\mathbf{c}_{p}(f)$ is a $\lambda$-adic unit.

(2) The complex number $\Omega_{f, N^{-}}$is given by

$$
\Omega_{f, N^{-}}=\frac{(4 \pi)^{k}\|f\|_{\Gamma_{0}(N)}}{\xi_{f}\left(N^{+}, N^{-}\right)}
$$

where $\|f\|_{\Gamma_{0}(N)}$ is the Petersson norm of $f$ and $\xi_{f}\left(N^{+}, N^{-}\right) \in \mathcal{O}_{f, \lambda}$ is an integer connected with certain congruence number of $f$. The precise definition is given in (4.3). It is interesting and important to make a comparison between $\Omega_{f, N^{-}}$and Hida's canonical period $\Omega_{f}$ attached to $f$. In general, we have $\Omega_{f, N^{-}} / \Omega_{f} \in \mathcal{O}_{f, \lambda}$. If $k=2$, then under a mild hypothesis, Pollack and Weston [PW11] have shown that this ratio is a product of local Tamagawa numbers at primes dividing $N^{-}$modulo a unit in $\mathcal{O}_{f, \lambda}$. We will investigate this subtle problem in $\$ 6$ for general weight $k$.

(3) Theorem $\mathrm{A}$ indeed gives the construction of the anticyclotomic $p$-adic $L$-function that interpolates square root of central $L$-values. In the case $k=2, \Theta_{\infty}$ is precisely the theta element $\theta_{\infty}$ (with trivial tame branch character) given by Bertolini and Darmon [BD96, p.436]. Therefore, combined with the anticyclotomic Iwasawa main conjecture for elliptic curves [BD05, the usual control theorem and the comparison between periods [PW11, Proposition 3.7, Theorem 6.8], the evaluation formula of $\Theta_{\infty}$ at the trivial character yields the optimal upper bound of the size of $p$-primary Selmer groups of certain elliptic curves over $K$ in terms of central $L$-values as predicted by Birch and Swinnerton-Dyer conjecture. 
Let $*: \mathcal{O}_{f, \lambda} \llbracket \Gamma^{-} \rrbracket \rightarrow \mathcal{O}_{f, \lambda} \llbracket \Gamma^{-} \rrbracket$ be the involution defined by $\sigma \mapsto \sigma^{-1}$. Then $\Theta_{\infty}$ satisfies the following functional equation.

Theorem B. Let $\sigma_{\mathfrak{N}^{+}}$be the image of $\mathfrak{N}^{+}$in $\Gamma^{-}$under the reciprocity law map $\operatorname{rec}_{K}$. Then we have the functional equation:

$$
\Theta_{\infty}^{*}=\epsilon_{p}(f) \epsilon(f) \cdot \Theta_{\infty} \cdot \sigma_{\mathfrak{N}^{+}}^{-1} .
$$

In the case $k=2$ and $\left(D_{K}, N^{-}\right)=1$, this theorem is proved in [BD96, Proposition 2.13] up to an element in $\Gamma^{-}$. The above functional equation suggests that the product $\left|\Theta_{\infty}\right|^{2}:=\Theta_{\infty} \cdot \Theta_{\infty}^{*}$ be intrinsic. Namely, $\left|\Theta_{\infty}\right|^{2}$ does not depend on the choice of an auxiliary decomposition $N^{+}=\mathfrak{N}^{+} \overline{\mathfrak{N}^{+}}$. Combined with Theorem $\mathrm{A}$. this verifies the formula of $\chi\left(\left|\Theta_{\infty}\right|^{2}\right)$ in [BD96. Conjecture 2.12]. If $\chi$ is ramified character of finite order and $p \nmid D_{K} N$, then the formula of $\chi\left(\left|\Theta_{\infty}\right|^{2}\right)$ actually is a consequence of Gross special value formulae first obtained by B. Gross in a special case $k=2$ and $N$ is a prime and generalized by Shou-Wu Zhang [Zha04, Theorem 7.1] for $k=2$ and Haiping Yuan Yua05, for $k>2$.

In [Vat03, Theorem 1.1], Vatsal determines the $\mu$-invariant of anticyclotomic $p$-adic $L$-functions for modular forms of weight two. Our second theorem provides a partial generalization of his result to modular forms of higher weight.

Theorem C. Let $\rho_{f, \lambda}: \operatorname{Gal}(\overline{\mathbf{Q}} / \mathbf{Q}) \rightarrow \mathrm{GL}_{2}\left(\mathcal{O}_{f, \lambda}\right)$ be the Galois representation associated to $f$. With the assumptions in Theorem $\AA$, suppose further

(1) $\left(D_{K}, N^{-}\right)=1$,

(2) the residual representation $\bar{\rho}_{f, \lambda}$ is absolutely irreducible.

Then the Iwasawa $\mu$-invariant of $\Theta_{\infty}$ vanishes.

Remark 1. Theorem Chas an important application to Iwasawa main conjecture for GL(2). Vatsal's theorem on the vanishing of $\mu$-invariant plays a key role in the proof of Iwasawa main conjecture for elliptic curves in the recent work of Skinner-Urban [SU10]. They actually prove the main conjecture for modular forms on $\Gamma_{0}(N)$ of weight $k \equiv 2(\bmod p-1)$ [SU10, Theorem 3.6.4], and Theorem Cenables us to lift their assumption $k \equiv 2(\bmod p-1)$.

Now we suppose that $\lambda$ has residue characteristic $\ell \neq p$ and consider the problem of non-vanishing modulo $\lambda$ of central $L$-values with anticyclotomic twists. We obtain the following result, which is a generalization and an improvement of [Vat03, Theorem 1.2] in the weight two case.

Theorem D. Suppose that $p^{2} \nmid N$ and $\left(D_{K}, N^{-}\right)=1$. Let $\ell$ be a rational prime such that

(1) $\ell \nmid p N D_{K}$ and $\ell>k-2$,

(2) $\bar{\rho}_{f, \lambda}$ is absolutely irreducible.

Then for all but finitely many characters $\chi: \Gamma^{-} \rightarrow \mu_{p^{\infty}}$, we have

$$
\frac{L(f / K, \chi, k / 2)}{\Omega_{f, N^{-}}} \not \equiv 0(\bmod \lambda) .
$$

Remark 2. Theorem $\mathrm{D}$ has several consequences in number theory and representation theory. In number theory, this theorem removes the assumptions on $p \nmid D_{K}$ and the $p$-indivisibility of the class number of $K$ in [Vat02, Theorem 1.4], and shows the finiteness of the $\ell$-primary Selmer groups of elliptic curves over $K_{\infty}^{-}$ in virtue of [LV10]. From representation theoretic point of view, this theorem provides a simultaneous nonvanishing result of central $L$-values with anticyclotomic twist, and hence has application to the non-vanishing of Bessel models of certain theta lifting on GSp(4) by [PTB11, Theorem 3].

The construction of the theta element $\Theta_{\infty}$ is based on an adelic formulation of the method of Bertolini and Darmon, with which one can borrow tools from representation theory (Such kind of adelic formulation was also used by Van Order [VO12] in the case of Hilbert modular forms of parallel weight two). The interpolation formula is the elaboration of an explicit Waldspurger formula combined with a $p$-adic congruence argument. We briefly describe these ideas in what follows. Let $B$ be the definite quaternion algebra over $\mathbf{Q}$ of the absolute discriminant $N^{-}$and let $R$ be an Eichler order of level $N / N^{-}$. Let $\varphi_{f}: B^{\times} \backslash B_{\mathbf{A}}^{\times} / \widehat{R}^{\times} \rightarrow \operatorname{Sym}^{k-2}\left(\mathbf{C}^{2}\right)$ be a vector-valued automorphic new form on $B$ attached to $f$ via Jacquet-Langlands correspondence. For each positive integer $n$, let $\mathcal{O}_{n}=\mathbf{Z}+p^{n} \mathcal{O}_{K}$ be the order of $K$ of conductor $p^{n}$ and let $\mathcal{G}_{n}=\operatorname{Gal}\left(H_{n} / K\right)$ be the Galois 
group of the ring-class field of $K$ of conductor $p^{n}$. Then the Picard group Pic $\mathcal{O}_{n}$ will be called Gross points of level $p^{n}$, which is a homogeneous space of $\mathcal{G}_{n}$. Our hypothesis on $N$ assures that there exists an optimal embedding $\iota_{n}: K \rightarrow B$ with respect to $\left(\mathcal{O}_{n}, R\right)$, which in turn induces a map $\iota_{n}: \operatorname{Pic} \mathcal{O}_{n} \rightarrow B^{\times} \backslash B_{\mathbf{A}}^{\times} / \widehat{R}^{\times}$. Fix a distinguished point $P_{n} \in \operatorname{Pic} \mathcal{O}_{n}$ and let $P_{n}^{\dagger}$ be the regularized Gross point (See $\$ 4.2$ ). The module $\operatorname{Sym}^{k-2}\left(\mathcal{O}_{f, \lambda}^{2}\right)$ has a natural $\mathcal{O}_{f, \lambda}$-basis $\left\{\mathbf{v}_{j}\right\}$ indexed by integers $-k / 2<j<k / 2$ (See (2.10) ), and we can write

$$
\varphi_{f}=\sum_{-k / 2<j<k / 2} \varphi_{f}^{[j]} \otimes \mathbf{v}_{j} .
$$

Here $\varphi_{f}^{[j]}: B^{\times} \backslash B_{\mathbf{A}}^{\times} \rightarrow \mathbf{C}$ are automorphic forms on $B_{\mathbf{A}}^{\times}$. One can take $p$-adically optimal normalization of $\varphi_{f}^{[0]}$ using the integral structure $\operatorname{Sym}^{k-2}\left(\mathcal{O}_{f, \lambda}^{2}\right)$ (see 4 .1.1). Moreover, under the ordinary assumption, it can be shown that the restriction of the normalized $\varphi_{f}^{[0]}$ to regularized Gross points does take value in $\mathcal{O}_{f, \lambda}$. We can thus define

$$
\widetilde{\Theta}_{n}=\sum_{\sigma \in \mathcal{G}_{n}} \sigma \otimes \varphi_{f}^{[0]}\left(\iota_{n}\left(\sigma\left(P_{n}^{\dagger}\right)\right)\right) \in \mathcal{O}_{f, \lambda}\left[\mathcal{G}_{n}\right] .
$$

Then $\left\{\widetilde{\Theta}_{n}\right\}_{n}$ is compatible with respect to the natural quotient $\mathcal{G}_{n+1} \rightarrow \mathcal{G}_{n}$. Then we obtain $\widetilde{\Theta}_{\infty}$ by taking the limit $\left\{\widetilde{\Theta}_{n}\right\}_{n} \in \mathcal{O}_{f, \lambda} \llbracket \mathcal{G}_{\infty} \rrbracket$, where $\mathcal{G}_{\infty}=\lim _{n} \mathcal{G}_{n}$. The Galois group $\Gamma^{-}=\operatorname{Gal}\left(K_{\infty}^{-} / K\right)$ is the maximal $\mathbf{Z}_{p}$-free quotient of $\mathcal{G}_{\infty}$. The theta element $\Theta_{\infty}$ in Theorem $\mathrm{A}$ is defined to be the projection of $\widetilde{\Theta}_{\infty}$ obtained by the quotient map $\mathcal{G}_{\infty} \rightarrow \Gamma^{-}$. If $\chi$ is a finite order character of conductor $p^{n}$, the evaluation of $\widehat{\chi}\left(\Theta_{\infty}\right)^{2}$ indeed can be translated into an explicit Waldspurger's formula. Let $\varphi_{f}^{\dagger}$ be the $p$-stabilization of $\varphi_{f}^{[0]}$ with respect to $A_{p}$. Then $\widehat{\chi}\left(\Theta_{\infty}\right)$ is essentially the global toric period given by

$$
P\left(\varphi_{f}^{\dagger}, \chi\right)=\int_{K \times \mathbf{A}_{\mathbf{Q}}^{\times} \backslash \mathbf{A}_{K}^{\times}} \varphi_{f}^{\dagger}\left(\iota_{n}(t)\right) \chi(t) d t .
$$

The value $P\left(\varphi_{f}^{\dagger}, \chi\right)^{2}$ is a product of local toric period integrals by the fundamental formula of Waldspurger Wal85, Proposition 7]. We make an explicit calculation of these local integrals. The new input is the calculation of the local toric integral of the $p$-stabilized local new vector at $p$. It is no surprise that the $p$-adic multiplier $e_{p}(f, \chi)$ is contributed by this local integral. Note that Waldspurger's formula only computes $\widehat{\chi}\left(\Theta_{\infty}\right)$ for finite order characters $\chi$. We obtain the formula of $\widehat{\chi}\left(\Theta_{\infty}\right)$ for characters $\widehat{\chi} \in \mathfrak{X}_{p}^{\text {crit }}$ of infinite order by a congruence trick (Corollary 4.5).

The proof of Theorem $\mathrm{C}$ is based on the uniform distribution of CM points in the zero dimensional Shimura variety attached to the definite quaternion algebra $B$, which is the idea of Vatsal in his study on the nonvanishing of anticyclotomic central $L$-values of weight two modular forms. In the higher weight situation, the new idea is to use the congruences among modular forms. Roughly speaking, we construct a weight two $\overline{\mathbb{F}}_{p}$-valued modular form $\mathbf{f}_{p}$ such that the evaluations of $\mathbf{f}_{p}$ and $\varphi_{f}^{[0]}$ at Gross points are congruent to each other. We thus reduce the problem to $\mathbf{f}_{p}$, for which the approach of Vatsal can be applied. Since the form $\mathbf{f}_{p}$ is not a new form in general, we have to use a stronger uniform distribution result [CV05, Proposition 2.10] and slightly generalized Ihara's lemma (Lemma [5.5). The proof of Theorem $\mathrm{D}$ is based on the same idea combined with a Galois average trick.

This paper is organized as follows. After fixing basic notation and definitions in \$1, we give a brief review of modular forms on definite quaternion algebras and an adelic description of Gross points in \$2 In \$3, we give the explicit calculation of the toric periods of $p$-stabilized modular forms based on Waldspurger's formula (Proposition 3.5). The calculation of the local toric integral at $p$ is carried out in Proposition 3.10, and the final formula is summarized in Theorem 3.11. In \$4 we give the construction of theta elements (Defefinition 4.1). The functional equation is proved in Theorem 4.8, and the evaluation formula Theorem 4.6 (Theorem $\mathrm{A}$ ) is obtained by combining Proposition 4.3 and the congruence property Corollary 4.5 among theta elements. In \$5. after preparing a key result of Vatsal-Cornut on the uniform distribution of CM points and Ihara's Lemma, we prove Theorem $\mathrm{C}$ (Theorem 5.7) and Theorem $\mathrm{D}$ (Theorem 5.9). Finally, in $\$ 6$ we give a sufficient condition (Proposition 6.1) under which the complex number $\Omega_{f, N^{-}}$equals Hida's canonical periods $\Omega_{f}$ up to a unit in $\mathcal{O}_{f, \lambda}$, applying techniques of Wiles, Taylor-Wiles and Diamond in their proofs of modularity lifting theorems. 
Acknowledgments. This project was initiated when the first author visited Taida Institute of Mathematical Science. He would like to thank for their hospitality.

\section{Notation AND DEFinitions}

1.1. If $L$ is a number field, $\mathcal{O}_{L}$ is the ring of integers of $L, \mathbf{A}_{L}$ is the adele of $L$ and $\mathbf{A}_{L, f}$ is the finite part of $\mathbf{A}_{L}$. Let $\mathbf{A}=\mathbf{A}_{\mathbf{Q}}$. Let $\psi=\prod \psi_{q}$ be the standard additive character of $\mathbf{A} / \mathbf{Q}$ such that $\psi\left(x_{\infty}\right)=$ $\exp \left(2 \pi i x_{\infty}\right), x_{\infty} \in \mathbf{R}$.

We fix once and for all an embedding $\iota_{\infty}: \overline{\mathbf{Q}} \hookrightarrow \mathbf{C}$ and an isomorphism $\iota: \mathbf{C} \simeq \mathbf{C}_{\ell}$ for each rational prime $\ell$, where $\mathbf{C}_{\ell}$ is the completion of an algebraic closure of $\mathbf{Q}_{\ell}$. Let $\iota_{\ell}=\iota_{\infty}: \overline{\mathbf{Q}} \hookrightarrow \mathbf{C}_{\ell}$ be their composition. Let $\operatorname{ord}_{\ell}: \mathbf{C}_{\ell} \rightarrow \mathbf{Q} \cup\{\infty\}$ be the $\ell$-adic valuation on $\mathbf{C}_{\ell}$ normalized so that $\operatorname{ord}_{\ell}(\ell)=1$. We regard $L$ as a subfield in $\mathbf{C}\left(\right.$ resp. $\left.\mathbf{C}_{\ell}\right)$ via $\iota_{\infty}\left(\operatorname{resp} . \iota_{\ell}\right)$ and $\operatorname{Hom}(L, \overline{\mathbf{Q}})=\operatorname{Hom}\left(L, \mathbf{C}_{\ell}\right)$.

Let $\overline{\mathbf{Z}}$ be the ring of algebraic integers of $\overline{\mathbf{Q}}$ and let $\overline{\mathbf{Z}}_{\ell}$ be the $\ell$-adic completion of $\overline{\mathbf{Z}}$ in $\mathbf{C}_{\ell}$. Denote by $\widehat{\mathbf{Z}}$ the finite completion of $\mathbf{Z}$. For an abelian group $M$, let $\widehat{M}:=M \otimes \mathbf{z} \widehat{\mathbf{Z}}$.

1.2. Measures on local fields. We fix some general notation and conventions on local fields used in $₫ 3$ Let $q$ be a place of $\mathbf{Q}$ and $|\cdot|_{\mathbf{Q}_{q}}$ be the standard absolute value on $\mathbf{Q}_{q}$. Let $F$ be a finite extension of $\mathbf{Q}_{q}$. If $F$ is non-archimedean, we usually denote by $\varpi_{F}$ a uniformizer of $F$. Denote by $\mathcal{O}_{F}$ the ring of integers of $F$. Let $D_{F}$ be the discriminant of $F / \mathbf{Q}_{q}$. Let $|\cdot|_{F}$ be the absolute value of $F$ normalized by $|x|_{F}=\left|\mathrm{N}_{F / \mathbf{Q}_{q}}(x)\right|_{\mathbf{Q}_{q}}$. We often simply write $|x|=|x|_{F}$ for $x \in F$ if its meaning is clear from the context without possible confusion. Let $\psi: \mathbf{A} / \mathbf{Q} \rightarrow \mathbf{C}^{\times}$be the additive character such that $\psi\left(x_{\infty}\right)=\exp (2 \pi i x)$. Let $\psi_{q}$ be the local component of $\psi$ at $q$ and let $\psi_{F}:=\psi_{q} \circ \mathrm{T}_{F / \mathbf{Q}_{q}}$, where $\mathrm{T}_{F / \mathbf{Q}_{q}}$ is the trace from $F$ to $\mathbf{Q}_{q}$.

Let $d x$ be the Haar measure on $F$ self-dual with respect to the pairing $\left(x, x^{\prime}\right) \mapsto \psi_{F}\left(x x^{\prime}\right)$. If $F$ is nonarchimedean, then $\operatorname{vol}\left(\mathcal{O}_{F}, d x\right)=\left|D_{F}\right|_{\mathbf{Q}_{q}}^{\frac{1}{2}}$. We recall the definition of the local zeta function $\zeta_{F}(s)$. If $F$ is non-archimedean, then

$$
\zeta_{F}(s)=\frac{1}{1-\left|\varpi_{F}\right|_{F}^{s}}
$$

If $F$ is archimedean, then

$$
\zeta_{\mathbf{R}}(s)=\Gamma_{\mathbf{R}}(s):=\pi^{-s / 2} \Gamma(s / 2) ; \zeta_{\mathbf{C}}(s)=\Gamma_{\mathbf{C}}(s):=2(2 \pi)^{-s} \Gamma(s) .
$$

The Haar measure $d^{\times} x$ on $F^{\times}$is normalized by

$$
d^{\times} x=\zeta_{F}(1)|x|_{F}^{-1} d x .
$$

In particular, if $F=\mathbf{R}$, then $d x$ is the Lebesgue measure and $d^{\times} x=|x|_{\mathbf{R}}^{-1} d x$, and if $F=\mathbf{C}$, then $d x$ is twice the Lebesgue measure on $\mathbf{C}$ and $d^{\times} x=2 \pi^{-1} r^{-1} d r d \theta\left(x=r e^{i \theta}\right)$.

1.3. $L$-functions. Let $F$ be a non-archimedean local field. Let $\pi$ be an irreducible admissible representation of $\mathrm{GL}_{2}(F)$. Let $L(s, \pi)$ and $\epsilon\left(s, \pi, \psi_{F}\right)$ be the associated local $L$-function and local epsilon factor respectively ([JL70, Theorem 2.18 (iv)]).

Let $E$ be a quadratic extension of $F$. We write $\pi_{E}$ for the base change of $\pi$. Let $\mu, \nu: F^{\times} \rightarrow \mathbf{C}^{\times}$be two characters of $F^{\times}$. Suppose that either $\pi=\pi(\mu, \nu)$ is a principal series if $\mu \nu^{-1} \neq|\cdot|^{ \pm 1}$ or $\pi=\sigma(\mu, \nu)$ is a special representation if $\mu \nu^{-1}=|\cdot|$. Let $\chi: E^{\times} \rightarrow \mathbf{C}^{\times}$be a character. We recall the definition of local $L$-functions $L\left(s, \pi_{E} \otimes \chi\right)\left(\left[\right.\right.$ Jac72, §20]). If $E=F \oplus F$, then we write $\chi=\left(\chi_{1}, \chi_{2}\right): F^{\times} \oplus F^{\times} \rightarrow \mathbf{C}^{\times}$and put

$$
L\left(s, \pi_{E} \otimes \chi\right)= \begin{cases}L\left(s, \pi \otimes \chi_{1}\right) L\left(s, \pi \otimes \chi_{2}\right) & \text { if } \mu \nu^{-1} \neq|\cdot|^{ \pm 1}, \\ L\left(s, \mu \chi_{1}\right) L\left(s, \mu \chi_{2}\right) & \text { if } \mu \nu^{-1}=|\cdot| .\end{cases}
$$

If $E$ is a field, then

$$
L\left(s, \pi_{E} \otimes \chi\right)= \begin{cases}L\left(s, \mu^{\prime} \chi\right) L\left(s, \nu^{\prime} \chi\right) & \text { if } \mu \nu^{-1} \neq|\cdot|^{ \pm 1}, \\ L\left(s, \mu^{\prime} \chi\right) & \text { if } \mu \nu^{-1}=|\cdot| .\end{cases}
$$

Here $\mu^{\prime}=\mu \circ \mathrm{N}_{E / F}, \nu^{\prime}=\nu \circ \mathrm{N}_{E / F}$ are characters of $E^{\times}$. 
1.4. Whittaker functions on $\mathrm{GL}_{2}\left(\mathbf{Q}_{q}\right)$. Let $q$ be a place of $\mathbf{Q}$ and let $\pi$ be an admissible irreducible representation of $\mathrm{GL}_{2}\left(\mathbf{Q}_{q}\right)$ with the trivial central character. Let $\mathcal{W}(\pi, \psi)$ be the Whittaker model of $\pi$ attached to the additive character $\psi=\psi_{q}: \mathbf{Q}_{q} \rightarrow \mathbf{C}^{\times}$. Recall that $\mathcal{W}(\pi, \psi)$ is a subspace of smooth functions $W: \mathrm{GL}_{2}(F) \rightarrow \mathbf{C}$ such that

(1) $W\left(\left(\begin{array}{ll}1 & x \\ 0 & 1\end{array}\right) g\right)=\psi(x) W(g)$ for all $x \in \mathbf{Q}_{q}$.

(2) If $q$ is the archimedean place, $W\left(\left(\begin{array}{ll}a & 0 \\ 0 & 1\end{array}\right)\right)=O\left(|a|^{M}\right)$ for some positive number $M$.

If $\mathbf{Q}_{q}$ is non-archimedean and $N$ is a positive integer, we put

$$
U_{0}(N)_{q}=\left\{g=\left(\begin{array}{ll}
a & b \\
c & d
\end{array}\right) \in \mathrm{GL}_{2}\left(\mathbf{Z}_{q}\right) \mid c \in N \mathbf{Z}_{q}\right\} .
$$

Let $c$ be the conductor of $\pi$. Let $W_{\pi}$ be the normalized Whittaker new form characterized by $W_{\pi}(1)=1$ and $W_{\pi}(g u)=W_{\pi}(g)$ for all $u \in U_{0}(c)_{q}$. If $\mathbf{Q}_{q}=\mathbf{R}$ and $\pi$ is a discrete series of weight $k$, then the normalized Whittaker new form $W_{\pi} \in \mathcal{W}(\pi, \psi)$ is defined by

$$
\begin{gathered}
W_{\pi}\left(z\left(\begin{array}{cc}
a & x \\
0 & 1
\end{array}\right)\left(\begin{array}{cc}
\cos \theta & \sin \theta \\
-\sin \theta & \cos \theta
\end{array}\right)\right)=a^{\frac{k}{2}} e^{-2 \pi a} \mathbb{I}_{\mathbf{R}_{+}}(a) \cdot \operatorname{sgn}(z)^{k} \psi(x) e^{i k \theta} \\
\left(a, z \in \mathbf{R}^{\times}, x, \theta \in \mathbf{R}\right) .
\end{gathered}
$$

Here $\mathbb{I}_{\mathbf{R}_{+}}(a)$ denotes the characteristic function of the set of positive real numbers. Recall that the zeta integral $\Psi(s, W, \chi)$ for $W \in \mathcal{W}(\pi, \psi)$ and a character $\chi: \mathbf{Q}_{q}^{\times} \rightarrow \mathbf{C}^{\times}$is defined by

$$
\Psi(s, W, \chi)=\int_{\mathbf{Q}_{q}^{\times}} W\left(\left(\begin{array}{cc}
a & 0 \\
0 & 1
\end{array}\right)\right) \chi(a)|a|^{s-\frac{1}{2}} d^{\times} a \quad(s \in \mathbf{C}) .
$$

Then $\Psi(s, W, \chi)$ converges absolutely for $\operatorname{Re} s \gg 0$ and has meromorphic continuation to the whole $s \in \mathbf{C}$.

Let $\mathcal{K}(\pi, \psi)$ be the Kirillov model of $\pi$ with respective to $\psi$. Then $\mathcal{K}(\pi, \psi)$ is a subspace of smooth functions $\phi: \mathbf{Q}_{q}^{\times} \rightarrow \mathbf{C}$, and there is an isomorphism $\mathcal{W}(\pi, \psi) \stackrel{\sim}{\rightarrow} \mathcal{K}(\pi, \psi)$ given by

$$
W \mapsto \phi_{W}(a):=W\left(\left(\begin{array}{cc}
a & 0 \\
0 & 1
\end{array}\right)\right) .
$$

We call $\phi_{W_{\pi}}$ the normalized Kirillow new form. By the list of Kirillov new forms [Sch02, §2.4], we can verify that

$$
\Psi\left(s, W_{\pi}, \chi\right)=L(s, \pi \otimes \chi) \text { for unramified character } \chi .
$$

\section{Gross points and modular forms on Definite QuATERnion Algebras}

2.1. Let $K$ be an imaginary quadratic field with the discriminant $-D_{K}<0$ and let $\delta=\sqrt{-D_{K}}$. Write $z \mapsto \bar{z}$ for the complex conjugation on $K$. Define $\boldsymbol{\theta} \in K$ by

$$
\boldsymbol{\theta}=\frac{D^{\prime}+\delta}{2}, D^{\prime}= \begin{cases}D_{K} & \text { if } 2 \nmid D_{K}, \\ D_{K} / 2 & \text { if } 2 \mid D_{K} .\end{cases}
$$

Then $\mathcal{O}_{K}=\mathbf{Z}+\mathbf{Z} \cdot \boldsymbol{\theta}$ and $\boldsymbol{\theta} \overline{\boldsymbol{\theta}}$ is a local uniformizer of primes that are ramified in $K$. Fix positive integers $N^{+}$ that are only divisible by prime split in $K$ and $N^{-}$that are only divisible by primes inert or ramified in $K$.

We assume that

$$
N^{-} \text {is the square-free product of an odd number of primes. }
$$

Let $B$ be the definite quaternion over $\mathbf{Q}$ which is ramified precisely at the prime factors of $N^{-}$and the archimedean place. We can regard $K$ as a subalgebra of $B$. Write $\mathrm{T}$ and $\mathrm{N}$ for the reduced trace and norm of $B$ respectively. Let $G=B^{\times}$be the algebraic group over $\mathbf{Q}$ and let $Z=\mathbf{Q}^{\times}$be the center of $G$. Fix a distinguished rational prime $p$ such that

$$
p \nmid N^{+} N^{-} .
$$

Let $\mathfrak{p}$ be the prime of $K$ above $p$ induced by $\iota_{p}: K \hookrightarrow \mathbf{C}_{p}$. Let $\ell \nmid N^{-}$be a rational prime $(\ell$ can be $p$ ). We choose a basis of $B=K \oplus K \cdot J$ over $K$ such that

- $J^{2}=\beta \in \mathbf{Q}^{\times}$with $\beta<0$ and $J t=\bar{t} J$ for all $t \in K$. 
- $\beta \in\left(\mathbf{Z}_{q}^{\times}\right)^{2}$ for all $q \mid p \ell N^{+}$and $\beta \in \mathbf{Z}_{q}^{\times}$for $q \mid D_{K}$.

Fix a square root $\sqrt{\beta} \in \overline{\mathbf{Q}}$ of $\beta$. We fix an isomorphism $i=\prod i_{q}: \widehat{B}^{\left(N^{-}\right)} \simeq M_{2}\left(\mathbf{A}_{f}^{\left(N^{-}\right)}\right)$as follows. For each finite place $q \mid p \ell N^{+}$, the isomorphism $i_{q}: B_{q} \simeq M_{2}\left(\mathbf{Q}_{q}\right)$ is defined by

$$
i_{q}(\boldsymbol{\theta})=\left(\begin{array}{cc}
\mathrm{T}(\boldsymbol{\theta}) & -\mathrm{N}(\boldsymbol{\theta}) \\
1 & 0
\end{array}\right) ; \quad i_{q}(J)=\sqrt{\beta} \cdot\left(\begin{array}{cc}
-1 & \mathrm{~T}(\boldsymbol{\theta}) \\
0 & 1
\end{array}\right) \quad\left(\sqrt{\beta} \in \mathbf{Z}_{q}^{\times}\right) .
$$

For each finite place $q \nmid p \ell N$, the isomorphism $i_{q}: B_{q}:=B \otimes_{\mathbf{Q}} \mathbf{Q}_{q} \simeq M_{2}\left(\mathbf{Q}_{q}\right)$ is chosen so that

$$
i_{q}\left(\mathcal{O}_{K} \otimes \mathbf{Z}_{q}\right) \subset M_{2}\left(\mathbf{Z}_{q}\right) .
$$

Hereafter, we shall identify $B_{q}$ and $G\left(\mathbf{Q}_{q}\right)$ with $M_{2}\left(\mathbf{Q}_{q}\right)$ and $\mathrm{GL}_{2}\left(\mathbf{Q}_{q}\right)$ via $i_{q}$ for finite $q \nmid N^{-}$. Finally, we define

$$
\begin{aligned}
& i_{K}: B \hookrightarrow M_{2}(K) \\
& a+b J \mapsto i_{K}(a+b J):=\left(\begin{array}{cc}
a & b \beta \\
\bar{b} & \bar{a}
\end{array}\right) \quad(a, b \in K)
\end{aligned}
$$

and let $i_{\mathbf{C}}: B \rightarrow M_{2}(\mathbf{C})$ be the composition $i_{\mathbf{C}}=\iota_{\infty} \circ i_{K}$

2.2. Optimal embeddings and Gross points. Fix a decomposition $N^{+} \mathcal{O}_{K}=\mathfrak{N}^{+} \overline{\mathfrak{N}^{+}}$once and for all. For each finite place $q \nmid p$, we define $\varsigma_{q} \in G\left(\mathbf{Q}_{q}\right)$ as follows:

$$
\begin{aligned}
& \varsigma_{q}=1 \text { if } q \nmid p N^{+}, \\
& \varsigma_{q}=\delta^{-1}\left(\begin{array}{ll}
\boldsymbol{\theta} & \overline{\boldsymbol{\theta}} \\
1 & 1
\end{array}\right) \in \mathrm{GL}_{2}\left(K_{\mathfrak{q}}\right)=\mathrm{GL}_{2}\left(\mathbf{Q}_{q}\right) \text { if } q=\mathfrak{q} \overline{\mathfrak{q}} \text { is split with } \mathfrak{q} \mid \mathfrak{N}^{+} .
\end{aligned}
$$

For $g \in B$, we put

$$
\iota_{\varsigma_{q}}(g):=i_{q}^{-1}\left(\varsigma_{q}^{-1} i_{q}(g) \varsigma_{q}\right) .
$$

If $q \mid N^{+}$and $t=\left(t_{1}, t_{2}\right) \in K_{q}:=K \otimes_{\mathbf{Q}} \mathbf{Q}_{q}=K_{\mathfrak{q}} \oplus K_{\bar{q}}$, then

$$
\iota_{\varsigma_{q}}(t)=\left(\begin{array}{cc}
t_{1} & 0 \\
0 & t_{2}
\end{array}\right)
$$

For each non-negative integer $n$, we choose $\varsigma_{p}^{(n)} \in G\left(\mathbf{Q}_{p}\right)$ as follows. If $p=\mathfrak{p} \overline{\mathfrak{p}}$ splits in $K$, we put

$$
\varsigma_{p}^{(n)}=\left(\begin{array}{cc}
\boldsymbol{\theta} & -1 \\
1 & 0
\end{array}\right)\left(\begin{array}{cc}
p^{n} & 0 \\
0 & 1
\end{array}\right) \in \mathrm{GL}_{2}\left(K_{\mathfrak{p}}\right)=\mathrm{GL}_{2}\left(\mathbf{Q}_{p}\right)
$$

If $p$ is inert or ramified in $K$, then we put

$$
\varsigma_{p}^{(n)}=\left(\begin{array}{cc}
0 & 1 \\
-1 & 0
\end{array}\right)\left(\begin{array}{cc}
p^{n} & 0 \\
0 & 1
\end{array}\right) .
$$

Define $x_{n}: \mathbf{A}_{K}^{\times} \rightarrow G(\mathbf{A})$ by

$$
x_{n}(a):=a \cdot \varsigma^{(n)} \quad\left(\varsigma^{(n)}:=\varsigma_{p}^{(n)} \prod_{q \neq p} \varsigma_{q}\right) .
$$

This collection $\left\{x_{n}(a)\right\}_{a \in \mathbf{A}_{K}^{\times}}$of points is called Gross points of conductor $p^{n}$ associated to $K$.

Let $\mathcal{O}_{n}=\mathbf{Z}+p^{n} \mathcal{O}_{K}$ be the order of $K$ of conductor $p^{n}$. For each positive integer $M$ prime to $N^{-}$, we denote by $R_{M}$ the Eichler order of level $M$ with respect to the isomorphisms $\left\{i_{q}: B_{q} \simeq M_{2}\left(\mathbf{Q}_{q}\right)\right\}_{q \nmid N^{-}}$. It is not difficult to verify immediately that the inclusion map $K \hookrightarrow B$ is an optimal embedding of $\mathcal{O}_{n}$ into the Eichler order $B \cap \varsigma^{(n)} \widehat{R}_{M}\left(\varsigma^{(n)}\right)^{-1}$ if $\operatorname{ord}_{p}(M) \leq n$. In other words,

$$
\left(B \cap \varsigma^{(n)} \widehat{R}_{M}\left(\varsigma^{(n)}\right)^{-1}\right) \cap K=\mathcal{O}_{n} .
$$


2.3. Modular forms on definite quaternion algebras. Let $k \geq 2$ be an even integer. For a ring $A$, we denote by $L_{k}(A)=\operatorname{Sym}^{k-2}\left(A^{2}\right)$ the set of the set of homogeneous polynomials of degree $k-2$ with coefficients in $A$. We write

$$
L_{k}(A)=\bigoplus_{-\frac{k}{2}<m<\frac{k}{2}} A \cdot \mathbf{v}_{m} \quad\left(\mathbf{v}_{m}:=X^{\frac{k-2}{2}-m} Y^{\frac{k-2}{2}+m}\right) .
$$

We let $\rho_{k}: \mathrm{GL}_{2}(A) \rightarrow \operatorname{Aut}_{A} L_{k}(A)$ be the unitary representation defined by

$$
\rho_{k}(g) P(X, Y)=\operatorname{det}(g)^{-\frac{k-2}{2}} \cdot P((X, Y) g) \quad\left(P(X, Y) \in L_{k}(A)\right) .
$$

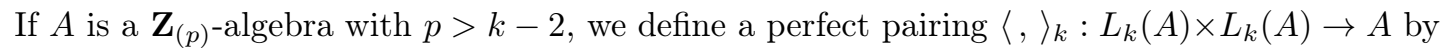

$$
\left\langle\sum_{i} a_{i} \mathbf{v}_{i}, \sum_{j} b_{j} \mathbf{v}_{j}\right\rangle_{k}=\sum_{-k / 2<m<k / 2} a_{m} b_{-m} \cdot(-1)^{\frac{k-2}{2}+m} \frac{\Gamma(k / 2+m) \Gamma(k / 2-m)}{\Gamma(k-1)} .
$$

This pairing is $\mathrm{GL}_{2}(A)$-equivariant, i.e. For $P, P^{\prime} \in L_{k}(A)$, we have

$$
\left\langle\rho_{k}(g) P, \rho_{k}(g) P^{\prime}\right\rangle_{k}=\left\langle P, P^{\prime}\right\rangle_{k} .
$$

Via the embedding $i_{\mathbf{C}}$ in (2.3), we obtain a representation

$$
\rho_{k, \infty}: G(\mathbf{R})=(B \otimes \mathbf{Q} \mathbf{R})^{\times} \stackrel{i_{\mathbf{C}}}{\longrightarrow} \mathrm{GL}_{2}(\mathbf{C}) \rightarrow \operatorname{Aut}_{\mathbf{C}} L_{k}(\mathbf{C}) .
$$

Then $\mathbf{C} \cdot \mathbf{v}_{m}$ is the eigenspace on which $\rho_{k, \infty}(t)$ acts by $(\bar{t} / t)^{m}$ for $t \in\left(K \otimes_{\mathbf{Q}} \mathbf{R}\right)^{\times}$. If $A$ is a $K$-algebra and $U \subset G\left(\mathbf{A}_{f}\right)$ is an open compact subgroup, we denote by $\mathbf{M}_{k}(U, A)$ be the space of modular forms of weight $k$ defined over $A$, consisting of functions $f: G\left(\mathbf{A}_{f}\right) \rightarrow L_{k}(A)$ such that

$$
f(\alpha g u)=\rho_{k, \infty}(\alpha) f(g) \text { for all } \alpha \in G(\mathbf{Q}), u \in U .
$$

The right translation makes $\mathbf{M}_{k}(A):=\lim _{U} \mathbf{M}_{k}(U, A)$ an admissible $G\left(\mathbf{A}_{f}\right)$-representation.

Let $\mathcal{A}(G)$ be the space of automorphic forms on $G(\mathbf{A})$. For $\mathbf{v} \in L_{k}(\mathbf{C})$ and $f \in \mathbf{M}_{k}(\mathbf{C})$, we define a function $\Psi(\mathbf{v} \otimes f): G(\mathbf{Q}) \backslash G(\mathbf{A}) \rightarrow \mathbf{C}$ by

$$
\Psi(\mathbf{v} \otimes f)(g):=\left\langle\rho_{k, \infty}\left(g_{\infty}\right) \mathbf{v}, f\left(g_{f}\right)\right\rangle_{k} .
$$

Then the map $\mathbf{v} \otimes f \mapsto \Psi(\mathbf{v} \otimes f)$ gives rise to $G(\mathbf{A})$-equivariant morphism $L_{k}(\mathbf{C}) \otimes \mathbf{M}_{k}(\mathbf{C}) \rightarrow \mathcal{A}(G)$. Let $\omega$ be a unitary Hecke character of $\mathbf{Q}$. We let

$$
\mathbf{M}_{k}(U, \omega, \mathbf{C})=\left\{f \in \mathbf{M}_{k}(U, \mathbf{C}) \mid f(z g)=\omega(z) f(g) \text { for all } z \in Z(\mathbf{A})\right\} .
$$

Let $\mathcal{A}_{k}(U, \omega, \mathbf{C})$ be the space of automorphic forms on $G(\mathbf{A})$ of weight $k$ and central character $\omega$, consisting of functions $\Psi(f \otimes \mathbf{v}): G(\mathbf{A}) \rightarrow \mathbf{C}$ for $f \in S_{k}(U, \omega, \mathbf{C})$ and $\mathbf{v} \in L_{k}(\mathbf{C})$. Denote by $\mathbf{1}$ the trivial character. For each positive integer $M$, we put

$$
\begin{aligned}
\mathbf{M}_{k}(M, \mathbf{C}) & =\mathbf{M}_{k}\left(\widehat{R}_{M}^{\times}, \mathbf{1}, \mathbf{C}\right), \\
\mathcal{A}_{k}(M, \mathbf{C}) & =\mathcal{A}_{k}\left(\widehat{R}_{M}^{\times}, \mathbf{1}, \mathbf{C}\right) .
\end{aligned}
$$

\section{Special value formula}

3.1. Global setting. Let $\pi$ be an unitary irreducible cuspidal automorphic representation on $\mathrm{GL}_{2}(\mathbf{A})$ with trivial central character. Henceforth, we make the following assumptions:

- The archimedean constituent $\pi_{\infty}$ is a discrete series of weight $k$;

- The conductor of $\pi$ is $N=p^{n_{p}} N^{+} N^{-}$;

- $\operatorname{ord}_{p}(N)=n_{p} \leq 1 \Longleftrightarrow p^{2} \nmid N$.

Let $\pi^{\prime}=\otimes \pi_{q}^{\prime}$ be the unitary irreducible cuspidal automorphic representation on $G(\mathbf{A})$ with trivial central character attached to $\pi$ via Jacquet-Langlands correspondence. Then we have

(1) The archimedean constituent $\pi_{\infty}^{\prime} \simeq\left(\rho_{k, \infty}, L_{k}(\mathbf{C})\right)$ as $G(\mathbf{R})$-modules, and $\pi_{q}^{\prime}$ is a unramified one dimensional representation for $q \mid N^{-}$.

(2) The local constituent $\pi_{p}^{\prime}=\pi_{p}$ is either an unramified principal series $\pi\left(\mu_{p}, \nu_{p}\right)$ or an unramified special representation $\sigma\left(\mu_{p}, \nu_{p}\right)$ with $\mu_{p} \nu_{p}^{-1}=|\cdot|_{\mathbf{Q}_{p}}$. 
3.2. $p$-stabilization of new forms. Let $\pi_{f}^{\prime}$ denote the finite constituent of $\pi^{\prime}$. Let

$$
N_{B}=p^{n_{p}} N^{+}=N / N^{-}
$$

and let $R:=R_{N_{B}}$ be the Eichler order of level $N_{B}$. The multiplicity one theorem together with our assumptions in particular imply that $\pi_{f}^{\prime}$ can be realized as a unique $G\left(\mathbf{A}_{f}\right)$-submodule $\mathbf{M}_{k}\left(\pi_{f}^{\prime}\right)$ of $\mathbf{M}_{k}(\mathbf{C})$ and $\mathbf{M}_{k}\left(N_{B}, \mathbf{C}\right)\left[\pi_{f}^{\prime}\right]:=\mathbf{M}_{k}\left(\pi_{f}^{\prime}\right) \cap \mathbf{M}_{k}\left(N_{B}, \mathbf{C}\right)$ is one dimensional. We fix a nonzero new form $f_{\pi^{\prime}} \in \mathbf{M}_{k}\left(N_{B}, \mathbf{C}\right)\left[\pi_{f}^{\prime}\right]$. Throughout this section, we fix an integer $m$ such that

$$
-k / 2<m<k / 2 \text {. }
$$

Define the automorphic form $\varphi_{\pi^{\prime}}^{[m]} \in \mathcal{A}_{k}\left(N_{B}, \mathbf{C}\right)$ by

$$
\varphi_{\pi^{\prime}}^{[m]}:=\Psi\left(\mathbf{v}_{m}^{*} \otimes f_{\pi^{\prime}}\right) \quad\left(\mathbf{v}_{m}^{*}=\sqrt{\beta}^{-m} D_{K}^{\frac{k-2}{2}} \cdot \mathbf{v}_{m}\right) .
$$

We shall simply write $\varphi_{\pi^{\prime}}$ for $\varphi_{\pi^{\prime}}^{[m]}$ for brevity. Set

$$
\alpha_{p}:=\mu_{p}(p)|p|_{p}^{-\frac{1}{2}} .
$$

Define the $p$-stabilization $f_{\pi^{\prime}}^{\dagger}$ with respect to $\alpha_{p}$ as follows: If $p \mid N$, let $f_{\pi^{\prime}}^{\dagger}=f_{\pi^{\prime}}$, and if $p \nmid N$, let

$$
f_{\pi^{\prime}}^{\dagger}=f_{\pi^{\prime}}-\frac{1}{\alpha_{p}} \cdot \pi^{\prime}\left(\left(\begin{array}{cc}
1 & 0 \\
0 & p
\end{array}\right)\right) f_{\pi^{\prime}}
$$

For $f \in \mathbf{M}_{k}\left(N_{B}, \mathbf{C}\right)$, recall that the $U_{p}$-operator on $f$ is defined by

$$
f \mid U_{p}(g)=\sum_{x \in \mathbf{Z} / p \mathbf{Z}} f\left(g\left(\begin{array}{ll}
p & x \\
0 & 1
\end{array}\right)\right) .
$$

Thus $f_{\pi^{\prime}}^{\dagger}$ is an $U_{p^{-}}$-eigenform with the eigenvalue $\alpha_{p}$. Let $\varphi_{\pi^{\prime}}^{\dagger}$ be the $p$-stabilization of $\varphi_{\pi^{\prime}}$ given by

$$
\varphi_{\pi^{\prime}}^{\dagger}:=\Psi\left(\mathbf{v}_{m}^{*} \otimes f_{\pi^{\prime}}^{\dagger}\right) \text {. }
$$

By definition, one can verify that

$$
\begin{aligned}
& \varphi_{\pi^{\prime}}^{\dagger}\left(x_{n}(\gamma a u)\right)=\varphi_{\pi^{\prime}}^{\dagger}\left(x_{n}\left(a_{f}\right)\right)\left(\bar{a}_{\infty} / a_{\infty}\right)^{m} \\
& \quad\left(\gamma \in K^{\times}, a=\left(a_{\infty}, a_{f}\right) \in \mathbf{C}^{\times} \times \widehat{K}^{\times}, u \in \widehat{\mathcal{O}}_{n}^{\times}\right) .
\end{aligned}
$$

3.3. The Petersson inner product of new forms on $\mathrm{GL}_{2}(\mathbf{A})$. For each place of $\mathbf{Q}$, recall that $W_{\pi_{q}}$ is the Whittaker new form normalized so that $W_{\pi_{q}}(1)=1$. Let $\varphi_{\pi}$ be the normalized new form in $\pi$. In other words,

$$
\varphi_{\pi}(g):=\sum_{\alpha \in \mathbf{Q}} W_{\pi}\left(\left(\begin{array}{cc}
\alpha & 0 \\
0 & 1
\end{array}\right) g\right) \quad\left(W_{\pi}=\prod_{q} W_{\pi_{q}}\right) .
$$

Let $\boldsymbol{\tau}^{N}=\prod_{q} \boldsymbol{\tau}_{q}^{N} \in \mathrm{GL}_{2}(\mathbf{A})$ be the Atkin-Lehner element defined by $\boldsymbol{\tau}_{\infty}^{N}=\left(\begin{array}{cc}1 & 0 \\ 0 & -1\end{array}\right)$ and $\boldsymbol{\tau}_{q}^{N}=\left(\begin{array}{cc}0 & 1 \\ -N & 0\end{array}\right)$ if $q \neq \infty$. Let $d^{t} g$ be the Tamagawa measure on $\mathrm{GL}_{2}$. We put

$$
\left\langle\varphi_{\pi}, \varphi_{\pi}\right\rangle_{\mathrm{GL}_{2}}:=\int_{\mathbf{A}^{\times} \mathrm{GL}_{2}(\mathbf{Q}) \backslash \mathrm{GL}_{2}(\mathbf{A})} \varphi_{\pi}(g) \varphi_{\pi}\left(g \boldsymbol{\tau}^{N}\right) d^{t} g .
$$

To give a formula of $\left\langle\varphi_{\pi}, \varphi_{\pi}\right\rangle_{\mathrm{GL}_{2}}$, we define the $\mathrm{GL}_{2}\left(\mathbf{Q}_{q}\right)$-equivariant pairing $\mathbf{b}_{q}: \mathcal{W}\left(\pi_{q}, \psi_{q}\right) \times \mathcal{W}\left(\pi_{q}, \psi_{q}\right) \rightarrow \mathbf{C}$ by

$$
\mathbf{b}_{q}\left(W_{1}, W_{2}\right):=\int_{\mathbf{Q}_{q}^{\times}} W_{1}\left(\left(\begin{array}{cc}
a & 0 \\
0 & 1
\end{array}\right)\right) W_{2}\left(\left(\begin{array}{cc}
-a & 0 \\
0 & 1
\end{array}\right)\right) d^{\times} a .
$$

The convergence of this integral follows from the fact that $\pi_{q}$ is the local constituent of a unitary cuspidal automorphic representation. Let $\left\|\varphi_{\pi}\right\|_{q}$ be the local norm of $\varphi_{\pi}$ at $q$ defined by

$$
\left\|\varphi_{\pi}\right\|_{q}:=\frac{\zeta_{\mathbf{Q}_{q}}(2)}{\zeta_{\mathbf{Q}_{q}}(1) L\left(1, \operatorname{Ad} \pi_{q}\right)} \cdot \mathbf{b}_{q}\left(W_{\pi_{q}}, \pi\left(\boldsymbol{\tau}_{q}^{N}\right) W_{\pi_{q}}\right) .
$$


It is not difficult to deduce from [Wal85, Proposition 5] that

$$
\left\langle\varphi_{\pi}, \varphi_{\pi}\right\rangle_{\mathrm{GL}_{2}}=\frac{2 L(1, \mathrm{Ad} \pi)}{\zeta_{\mathbf{Q}}(2)} \cdot \prod_{q}\left\|\varphi_{\pi}\right\|_{q} .
$$

Define the local root number $\epsilon\left(\pi_{q}\right)$ by

$$
\epsilon\left(\pi_{q}\right):=\epsilon\left(\frac{1}{2}, \pi_{q}, \psi_{q}\right) \in\{ \pm 1\}
$$

The following lemma is well-known.

Lemma 3.1. Let $q$ be a finite place. We have $\pi\left(\boldsymbol{\tau}_{q}^{N}\right) W_{\pi_{q}}=\epsilon\left(\pi_{q}\right) \cdot W_{\pi_{q}}$.

Proof. We suppress the subscript $q$ and write $\pi=\pi_{q}$ and $\psi=\psi_{q}$ for brevity. Since $\pi\left(\boldsymbol{\tau}_{q}^{N}\right) W_{\pi}$ is also a nonzero new vector of $\pi$, we find that $\pi\left(\boldsymbol{\tau}_{q}^{N}\right) W_{\pi}=C \cdot W_{\pi}$ for some constant $C \in \mathbf{C}^{\times}$. Recall that we have the local functional equation [JL70, Theorem 2.18 (iv)]:

$$
\frac{\Psi(1-s, \widehat{W}, \mathbf{1})}{L(1-s, \pi)}=\epsilon(s, \pi, \psi) \cdot \frac{\Psi(s, W, \mathbf{1})}{L(s, \pi)},
$$

where $\widehat{W}(g):=W\left(g\left(\begin{array}{cc}0 & 1 \\ -1 & 0\end{array}\right)\right) \in \mathcal{W}(\pi, \psi)$. To evaluate $C$, we compute the zeta integral:

$$
\begin{aligned}
C \cdot \Psi\left(s, W_{\pi}, \mathbf{1}\right) & =\Psi\left(s, \pi\left(\boldsymbol{\tau}_{q}^{N}\right) W_{\pi}, \mathbf{1}\right) \\
= & \int_{F^{\times}} \pi\left(\boldsymbol{\tau}_{q}^{N}\right) W_{\pi}\left(\left(\begin{array}{cc}
a & 0 \\
0 & 1
\end{array}\right)\right)|a|^{s-\frac{1}{2}} d^{\times} a \\
= & |N|^{s-\frac{1}{2}} \cdot \int_{F^{\times}} \pi\left(\left(\begin{array}{cc}
0 & 1 \\
-1 & 0
\end{array}\right)\right) W_{\pi}\left(\left(\begin{array}{ll}
a & 0 \\
0 & 1
\end{array}\right)\right)|a|^{s-\frac{1}{2}} d^{\times} a \\
= & |N|^{s-\frac{1}{2}} \cdot \Psi\left(s, \widehat{W}_{\pi}, \mathbf{1}\right) \\
= & |N|^{s-\frac{1}{2}} \cdot \epsilon(1-s, \pi, \psi) \frac{L(s, \pi)}{L(1-s, \pi)} \cdot \Psi\left(1-s, W_{\pi}, \mathbf{1}\right) .
\end{aligned}
$$

It follows from (1.1) that

This completes the proof.

$$
C=|N|^{s-\frac{1}{2}} \cdot \epsilon(1-s, \pi, \psi)=\epsilon\left(\frac{1}{2}, \pi, \psi\right)
$$

Lemma 3.2. We have $\left\|\varphi_{\pi}\right\|_{q}=1$ for finite $q \nmid N$ and $\left\|\varphi_{\pi}\right\|_{\infty}=2^{-k-1}$. If $q \mid N^{-}$, then

$$
\left\|\varphi_{\pi}\right\|_{q}=\epsilon\left(\pi_{q}\right) \cdot(1+|q|)^{-1} .
$$

Proof. The assertions for $q \nmid N$ and $q=\infty$ are straightforward. Suppose that $q \mid N^{-}$. Then $\pi=\pi_{q}$ is a unramified special representation, and $L(1, \operatorname{Ad} \pi)=\zeta_{\mathbf{Q}_{q}}(2)$. By Lemma 3.1 we have

$$
\begin{aligned}
\mathbf{b}_{q}\left(W_{\pi}, \pi\left(\boldsymbol{\tau}_{q}^{N}\right) W_{\pi}\right) & =\int_{F^{\times}} W_{\pi}\left(\left(\begin{array}{cc}
a & 0 \\
0 & 1
\end{array}\right)\right) \cdot \pi\left(\boldsymbol{\tau}_{q}^{N}\right) W_{\pi}\left(\left(\begin{array}{cc}
-a & 0 \\
0 & 1
\end{array}\right)\right) d^{\times} a \\
& =\epsilon\left(\frac{1}{2}, \pi, \psi\right) \cdot \int_{F^{\times}} W_{\pi}\left(\left(\begin{array}{cc}
a & 0 \\
0 & 1
\end{array}\right)\right) W_{\pi}\left(\left(\begin{array}{cc}
a & 0 \\
0 & 1
\end{array}\right)\right) d^{\times} a \\
& =\epsilon\left(\frac{1}{2}, \pi, \psi\right) L(1, \operatorname{Ad} \pi) .
\end{aligned}
$$

The Petersson inner product $\left\|\varphi_{\pi}\right\|_{\Gamma_{0}(N)}$ of $\varphi_{\pi}$ is defined by

$$
\left\|\varphi_{\pi}\right\|_{\Gamma_{0}(N)}:=\operatorname{vol}\left(U_{0}(N), d^{t} g\right)^{-1} \cdot \int_{\mathbf{A} \times \mathrm{GL}_{2}(\mathbf{Q}) \backslash \mathrm{GL}_{2}(\mathbf{A})}\left|\varphi_{\pi}(g)\right|^{2} d^{t} g,
$$

where $U_{0}(N)=\mathrm{O}(2, \mathbf{R}) \times \prod_{q<\infty} U_{0}(N)_{q}$. Note that

$$
\operatorname{vol}\left(U_{0}(N), d^{t} g\right)^{-1}=\zeta_{\mathbf{Q}}(2) N \prod_{q \mid N}\left(1+q^{-1}\right) .
$$


We have the following proposition:

Proposition 3.3 (Theorem 5.1 [Hid81]). We have

$$
L(1, \operatorname{Ad} \pi)=\left\|\varphi_{\pi}\right\|_{\Gamma_{0}(N)} \cdot 2^{k} N^{-1} \cdot \prod_{q \mid N_{B}} \frac{\epsilon\left(\pi_{q}\right)}{\left(1+q^{-1}\right)\left\|\varphi_{\pi}\right\|_{q}} .
$$

Proof. By (3.6) and Lemma 3.2, we have

$$
\left\langle\varphi_{\pi}, \varphi_{\pi}\right\rangle_{\mathrm{GL} 2}=\frac{2 L(1, \mathrm{Ad} \pi)}{\zeta_{\mathbf{Q}}(2)} \cdot 2^{-k-1} \prod_{q \mid N^{-}} \frac{\epsilon\left(\pi_{q}\right)}{1+q^{-1}} \cdot \prod_{q \mid N_{B}}\left\|\varphi_{\pi}\right\|_{q}
$$

On the other hand, it is well known that

$$
\varphi_{\pi}\left(g \tau^{N}\right)=\epsilon\left(\pi_{f}\right) \cdot \overline{\varphi_{\pi}(g)} \quad\left(\epsilon\left(\pi_{f}\right):=\prod_{q<\infty} \epsilon\left(\pi_{q}\right)\right)
$$

and hence

$$
\begin{aligned}
\left\langle\varphi_{\pi}, \varphi_{\pi}\right\rangle_{\mathrm{GL}_{2}} & =\epsilon\left(\pi_{f}\right) \cdot \operatorname{vol}\left(U_{0}(N), d^{t} g\right) \cdot\left\|\varphi_{\pi}\right\|_{\Gamma_{0}(N)} \\
& =\left\|\varphi_{\pi}\right\|_{\Gamma_{0}(N)} \cdot \frac{1}{N \zeta_{\mathbf{Q}}(2)} \cdot \prod_{q \mid N} \frac{\epsilon\left(\pi_{q}\right)}{1+q^{-1}} .
\end{aligned}
$$

Combining these formulae, we find that

$$
L(1, \operatorname{Ad} \pi)=2^{k} N^{-1}\left\|\varphi_{\pi}\right\|_{\Gamma_{0}(N)} \cdot \prod_{q \mid N_{B}} \frac{\epsilon\left(\pi_{q}\right)}{\left(1+q^{-1}\right)\left\|\varphi_{\pi}\right\|_{q}} .
$$

3.4. Local toric integrals. For each place $q$ of $\mathbf{Q}$, denote by $\pi_{q}^{\prime}$ the local constituent of $\pi^{\prime}$ at $q$.

Definition 3.4. Define the new vector $\varphi_{q} \in \pi_{q}^{\prime}$ as follows:

(a) if $q=\infty$, then $\varphi_{q}$ is a multiple of $\mathbf{v}_{m} \in L_{k}(\mathbf{C}) \simeq \pi_{\infty}^{\prime}$,

(b) if $q \mid N^{-}$, then $\varphi_{q}$ is a basis of the one dimensional representation $\pi_{q}^{\prime}$ of $G\left(\mathbf{Q}_{q}\right)$,

(c) if $q \nmid N^{-}$, then $\varphi_{q}$ is fixed by $\left(R \otimes \mathbf{z} \mathbf{Z}_{q}\right)^{\times} \simeq U_{0}(N)_{q}$.

Let $\varphi_{q}^{\dagger}=\varphi_{q}$ if either $q \neq p$ or $q=p \mid N$ and let

$$
\varphi_{q}^{\dagger}=\varphi_{q}-\frac{1}{\alpha_{p}} \cdot \pi\left(\left(\begin{array}{ll}
1 & 0 \\
0 & p
\end{array}\right)\right) \varphi_{q} \text { if } q=p \nmid N .
$$

Define the local Atkin-Lehner element $\tau_{q}^{N_{B}} \in G\left(\mathbf{Q}_{q}\right)$ as follows: $\tau_{q}^{N_{B}}=J$ for $q \mid \infty N^{-}, \tau_{q}^{N_{B}}=1$ for finite place $q \nmid N$ and $\tau_{q}^{N_{B}}=\left(\begin{array}{cc}0 & 1 \\ -N_{B} & 0\end{array}\right)$ if $q \mid N_{B}$. Let $\tau^{N_{B}}:=\prod \tau_{q}^{N_{B}} \in G(\mathbf{A})$. Since $\pi^{\prime}$ has trivial central character, $\pi_{q}^{\prime}$ is self-dual. Hence, there exists a non-degenerate $G\left(\mathbf{Q}_{q}\right)$-equivariant pairing $\langle,\rangle_{q}: \pi_{q}^{\prime} \times \pi_{q}^{\prime} \rightarrow \mathbf{C}$. This pairing is unique up to a nonzero scalar.

For $g \in G\left(\mathbf{Q}_{q}\right)$ and a character $\chi: K_{q}^{\times} \rightarrow \mathbf{C}^{\times}$, we define the local toric integral for the new vector $\varphi_{q}$ by

$$
\mathcal{P}\left(g, \varphi_{q}, \chi\right)=\frac{L\left(1, \operatorname{Ad} \pi_{q}\right) L\left(1, \tau_{K_{q} / \mathbf{Q}_{q}}\right)}{\zeta_{\mathbf{Q}_{q}}(2) L\left(\frac{1}{2}, \pi_{K_{q}} \otimes \chi\right)} \cdot \int_{K_{q}^{\times} / \mathbf{Q}_{q}^{\times}} \frac{\left\langle\pi^{\prime}(t g) \varphi_{q}^{\dagger}, \pi^{\prime}(J g) \varphi_{q}^{\dagger}\right\rangle_{q}}{\left\langle\varphi_{q}, \pi^{\prime}\left(\tau_{q}^{N_{B}}\right) \varphi_{q}\right\rangle_{q}} \cdot \chi\left(t_{q}\right) d t_{q},
$$

where $\tau_{K_{q} / \mathbf{Q}_{q}}$ denotes the quadratic character of $\mathbf{Q}_{q}^{\times}$associated to $K_{q} / \mathbf{Q}_{q}$ and $d t_{q}$ is the quotient measure of the Haar measures of $K_{q}^{\times}$and $\mathbf{Q}_{q}^{\times}$fixed in $\$ 1$ An important observation is that the number $\mathcal{P}\left(g, \varphi_{q}, \chi\right)$ does not depend on the choice of the pairing $\langle,\rangle_{q}$, depending only on $\chi$ and the line spanned by $\varphi_{q}$. 
3.5. Waldspurger's formula. Let $\chi: K^{\times} \backslash \mathbf{A}_{K}^{\times} \rightarrow \mathbf{C}^{\times}$be an anticyclotomic Hecke character of archimedean weight $(m,-m)$. Namely,

$$
\left.\chi\right|_{\mathbf{A}^{\times}}=\mathbf{1} \text { and } \chi_{\infty}(z)=\left(\frac{z}{\bar{z}}\right)^{m} \quad(-k / 2<m<k / 2) .
$$

Let $\pi_{K}$ be the automorphic representation on $\mathrm{GL}_{2}\left(\mathbf{A}_{K}\right)$ via the quadratic base change of $\pi$ and let $L\left(s, \pi_{K} \otimes \chi\right)$ be the automorphic $L$-function of $\pi_{K} \otimes \chi$, which satisfies the functional equation

$$
L\left(s, \pi_{K} \otimes \chi\right)=\epsilon\left(s, \pi_{K} \otimes \chi\right) L\left(1-s, \pi_{K}^{\vee} \otimes \chi^{-1}\right),
$$

where $\epsilon\left(s, \pi_{K} \otimes \chi\right)=\prod_{q} \epsilon\left(s, \pi_{K_{q}} \otimes \chi_{q}, \psi_{K_{q}}\right)$ is the product of local epsilon factors. Let $\mathcal{A}\left(\pi^{\prime}\right)$ be the automorphic realization of $\pi^{\prime}$ in $\mathcal{A}(G)$. For $\varphi \in \mathcal{A}\left(\pi^{\prime}\right)$ and $g \in G(\mathbf{A})$, define the global toric period integral by

$$
P(g, \varphi, \chi):=\int_{K^{\times} \mathbf{A}^{\times} \backslash \mathbf{A}_{K}^{\times}} \varphi(t g) \chi(t) d t,
$$

where $d t$ is the measure of $K^{\times} / \mathbf{Q}^{\times}$with the volume $\operatorname{vol}\left(K^{\times} \mathbf{A}^{\times} \backslash \mathbf{A}_{K}^{\times}, d t\right)=2 L\left(1, \tau_{K / \mathbf{Q}}\right)$, where $L\left(s, \tau_{K / \mathbf{Q}}\right)$ is the complete $L$-function of the quadratic character attached to $K / \mathbf{Q}$ (cf. [Wal85, p. 180]). For $\varphi_{1}, \varphi_{2} \in \mathcal{A}\left(\pi^{\prime}\right)$, we define the $G(\mathbf{A})$-equivariant pairing:

$$
\left\langle\varphi_{1}, \varphi_{2}\right\rangle_{G}=\int_{G(\mathbf{Q}) Z(\mathbf{A}) \backslash G(\mathbf{A})} \varphi_{1}(g) \varphi_{2}(g) d g,
$$

where $d g$ is the Tamagawa measure on $G / Z$. By the theory of new forms [Cas73, $\varphi_{\pi^{\prime}}$ is characterized uniquely up to a scalar by the equations $\pi^{\prime}\left(\widehat{R}^{\times}\right) \varphi_{\pi^{\prime}}=\varphi_{\pi^{\prime}}$ and $\pi_{\infty}^{\prime}(t) \varphi_{\pi^{\prime}}=(\bar{t} / t)^{m} \varphi_{\pi^{\prime}}$ for $t \in K^{\times}$, so we have $\pi^{\prime}\left(\tau^{N_{B}}\right) \varphi_{\pi^{\prime}}(g)=C \cdot \overline{\varphi_{\pi^{\prime}}(g)}$ for some constant $C \in \mathbf{C}^{\times}$. This in particular implies that

$$
\left\langle\varphi_{\pi^{\prime}}, \pi^{\prime}\left(\tau^{N_{B}}\right) \varphi_{\pi^{\prime}}\right\rangle_{G} \neq 0 .
$$

Since $\langle,\rangle_{G}$ is a nonzero multiple of the product $\otimes_{q}\langle,\rangle_{q}$, we have

$$
\left\langle\varphi_{q}, \pi^{\prime}\left(\tau_{q}^{N_{B}}\right) \varphi_{q}\right\rangle_{q} \neq 0 \text { for each place } q .
$$

We shall make use of the following version of Waldspurger's formula, which expresses the global toric period integral as a product of local toric integrals.

Proposition 3.5. We have

$$
\frac{P\left(\varsigma^{(n)}, \varphi_{\pi^{\prime}}^{\dagger}, \chi\right)^{2}}{\left\langle\varphi_{\pi^{\prime}}, \pi^{\prime}\left(\tau^{N_{B}}\right) \varphi_{\pi^{\prime}}\right\rangle_{G}}=\frac{\zeta_{\mathbf{Q}}(2)}{2 L(1, \operatorname{Ad} \pi)} \cdot L\left(\frac{1}{2}, \pi_{K} \otimes \chi\right) \cdot \prod_{q} \mathcal{P}\left(\varsigma_{q}^{(n)}, \varphi_{q}, \chi_{q}\right),
$$

where $q$ runs over all places of $\mathbf{Q}$.

Proof. Fix an isomorphism $i: \pi^{\prime} \simeq \otimes_{q} \pi_{q}^{\prime}$ such that $i\left(\varphi_{\pi^{\prime}}\right)=\otimes_{q} \varphi_{q}$ for $\varphi_{q}$ chosen in Defefinition 3.4. Set $\varphi_{1}=\pi^{\prime}\left(\varsigma^{(n)}\right) \varphi_{\pi^{\prime}}^{\dagger}, \varphi_{2}=\pi^{\prime}\left(J_{\varsigma}^{(n)}\right) \varphi_{\pi^{\prime}}^{\dagger}, \varphi_{3}=\varphi_{\pi^{\prime}}$ and $\varphi_{4}=\pi^{\prime}\left(\tau^{N_{B}}\right) \varphi_{\pi^{\prime}}$. Let $i\left(\varphi_{i}\right)=\otimes_{q} \varphi_{i, q}$. Let $\bar{\chi}$ be the character defined by $\bar{\chi}(t)=\chi(\bar{t})$. Note that $P\left(1, \varphi_{1}, \chi\right)=P\left(\varsigma^{(n)}, \varphi_{\pi^{\prime}}^{\dagger}, \chi\right)$ and $P\left(1, \varphi_{2}, \bar{\chi}\right)=P\left(\varsigma^{(n)}, \varphi_{\pi^{\prime}}^{\dagger}, \chi\right)$. It follows from Waldspurger's formulae [Wal85, Proposition 4, Proposition 5, Lemme 7] that if $\left\langle\varphi_{3}, \varphi_{4}\right\rangle_{G} \neq 0$, then

$$
\frac{P\left(1, \varphi_{1}, \chi\right) P\left(1, \varphi_{2}, \bar{\chi}\right)}{\left\langle\varphi_{3}, \varphi_{4}\right\rangle_{G}}=\frac{\zeta_{\mathbf{Q}}(2) L\left(\frac{1}{2}, \pi_{K} \otimes \chi\right)}{2 L(1, \operatorname{Ad} \pi)} \cdot \prod_{q} P_{q}\left(\varphi_{1}, \varphi_{2}, \varphi_{3}, \varphi_{4}, \chi\right),
$$

where

$$
P_{q}\left(\varphi_{1}, \varphi_{2}, \varphi_{3}, \varphi_{4}, \chi\right)=\frac{L\left(1, \mathrm{Ad} \pi_{q}\right) L\left(1, \tau_{K_{q} / \mathbf{Q}_{q}}\right)}{L\left(\frac{1}{2}, \pi_{K_{q}} \otimes \chi_{q}\right) \zeta_{\mathbf{Q}_{q}}(2)} \cdot \int_{K_{q}^{\times} / \mathbf{Q}_{q}^{\times}} \frac{\left\langle\pi^{\prime}\left(t_{q}\right) \varphi_{1, q}, \varphi_{2, q}\right\rangle_{q}}{\left\langle\varphi_{3, q}, \varphi_{4, q}\right\rangle_{q}} \cdot \chi\left(t_{q}\right) d t_{q} .
$$

Moreover, $P_{q}\left(\varphi_{1}, \varphi_{2}, \varphi_{3}, \varphi_{4}, \chi\right)=1$ for all but finitely many $q$. The proposition follows immediately. 
Let $C l(R)$ be a set of representatives of $B^{\times} \backslash \widehat{B}^{\times} / \widehat{R}^{\times} \widehat{\mathbf{Q}}^{\times}$in $\widehat{B}^{\times}=G\left(\mathbf{A}_{f}\right)$. Define the inner product of $f_{\pi^{\prime}}$ by

$$
\left\langle f_{\pi^{\prime}}, f_{\pi^{\prime}}\right\rangle_{R}:=\sum_{g \in C l(R)} \frac{1}{\sharp \Gamma_{g}} \cdot\left\langle f_{\pi^{\prime}}(g), f_{\pi^{\prime}}\left(g \tau^{N_{B}}\right)\right\rangle_{k} \quad\left(\Gamma_{g}:=\left(B^{\times} \cap g \widehat{R}^{\times} g^{-1} \widehat{\mathbf{Q}}^{\times}\right) / \mathbf{Q}^{\times}\right) .
$$

Let $\epsilon_{q}\left(\pi_{K}, \chi\right):=\epsilon\left(\frac{1}{2}, \pi_{K_{q}} \otimes \chi_{q}, \psi_{K_{q}}\right) \in\{ \pm 1\}$ be the local root number of $\pi_{K_{q}} \otimes \chi_{q}$.

Corollary 3.6. Suppose that $\chi$ is unramified outside $p$. Then we have

$$
\begin{aligned}
P\left(\varsigma^{(n)}, \varphi_{\pi^{\prime}}^{\dagger}, \chi\right)^{2} \cdot \frac{\left\|\varphi_{\pi}\right\|_{\Gamma_{0}(N)}}{\left\langle f_{\pi^{\prime}}, f_{\pi^{\prime}}\right\rangle_{R}}= & \frac{2^{2-k}(-1)^{m} D_{K}^{k-2}}{\sqrt{D_{K}}} \cdot L\left(\frac{1}{2}, \pi_{K} \otimes \chi\right) \cdot \prod_{q \mid\left(D_{K}, N^{-}\right)}\left(1-\epsilon_{q}\left(\pi_{K}, \chi\right)\right) \\
& \times \prod_{q \mid p N^{+}} \mathcal{P}\left(\varsigma_{q}, \varphi_{q}, \chi_{q}\right) \cdot \frac{\left\|\varphi_{\pi}\right\|_{q}}{\left|D_{K}\right|_{\mathbf{Q}_{q}}^{\frac{1}{2}} \epsilon\left(\pi_{q}\right)} .
\end{aligned}
$$

Proof. Let $v_{R}$ be the volume of $G(\mathbf{R}) \widehat{R}^{\times}$in $Z(\mathbf{A}) G(\mathbf{Q}) \backslash G(\mathbf{A})$ and $d g_{\infty}$ be the Haar measure on the compact group $G(\mathbf{R}) / Z(\mathbf{R})$ with the volume one. By Schur orthogonality relations, we have

$$
\begin{aligned}
\left\langle\varphi_{\pi^{\prime}}, \pi^{\prime}\left(\tau^{N_{B}}\right) \varphi_{\pi^{\prime}}\right\rangle_{G} & =\sum_{g \in C l(R)} \frac{1}{\sharp \Gamma_{g}} \cdot \int_{G(\mathbf{R}) / Z(\mathbf{R})}\left\langle\rho_{k, \infty}\left(g_{\infty}\right) \mathbf{v}_{m}^{*}, f_{\pi^{\prime}}(g)\right\rangle_{k} \cdot\left\langle\rho_{k, \infty}\left(g_{\infty} J\right) \mathbf{v}_{m}^{*}, f_{\pi^{\prime}}\left(g \tau_{f}^{N_{B}}\right)\right\rangle_{k} d g_{\infty} \cdot v_{R} \\
& =\sum_{g \in C l(R)} \frac{1}{\sharp \Gamma_{g}} \cdot\left\langle f_{\pi^{\prime}}(g), f\left(g \tau^{N_{B}}\right)\right\rangle_{k} \cdot \frac{\left\langle\mathbf{v}_{m}^{*}, \rho_{k, \infty}(J) \mathbf{v}_{m}^{*}\right\rangle_{k}}{\operatorname{dim} L_{\mathbf{C}} L_{k-2}(\mathbf{C})} \cdot v_{R} \\
& =\left\langle f_{\pi^{\prime}}, f_{\pi^{\prime}}\right\rangle_{R} \cdot \frac{(-1)^{\frac{2-k}{2}} D_{K}^{k-2}\left\langle\mathbf{v}_{m}, \mathbf{v}_{k-m-2}\right\rangle_{k}}{(k-1)} \cdot v_{R},
\end{aligned}
$$

By the Eichler mass formula:

$$
\sum_{g \in C l(R)} \frac{1}{\sharp \Gamma_{g}}=\frac{\zeta_{\mathbf{Q}}(2)}{4 \pi} \cdot N \prod_{q \mid N^{-}}\left(1-q^{-1}\right) \prod_{q \mid N_{B}}\left(1+q^{-1}\right),
$$

and $\operatorname{vol}(Z(\mathbf{A}) G(\mathbf{Q}) \backslash G(\mathbf{A}), d g)=2$, we find that

$$
v_{R}=\frac{8 \pi}{\zeta_{\mathbf{Q}}(2) N} \cdot \prod_{q \mid N^{-}} \zeta_{\mathbf{Q}_{q}}(1) \prod_{q \mid N_{B}}\left(1+q^{-1}\right)^{-1},
$$

and hence

$$
\left\langle\varphi_{\pi^{\prime}}, \pi^{\prime}\left(\tau^{N_{B}}\right) \varphi_{\pi^{\prime}}\right\rangle_{G}=\left\langle f_{\pi^{\prime}}, f_{\pi^{\prime}}\right\rangle_{R} \cdot \frac{(-1)^{m} D_{K}^{k-2} \Gamma(k / 2+m) \Gamma(k / 2-m)}{\Gamma(k)} \cdot \frac{8 \pi}{\zeta_{\mathbf{Q}}(2) N} \cdot \prod_{q \mid N^{-}} \zeta_{\mathbf{Q}_{q}}(1) \prod_{q \mid N_{B}}\left(1+q^{-1}\right)^{-1} .
$$

Combining with Proposition 3.3. Proposition 3.5 and (3.10), we find that

$$
\begin{aligned}
P\left(\varsigma^{(n)}, \varphi_{\pi^{\prime}}^{\dagger}, \chi\right)^{2} \cdot \frac{\left\|\varphi_{\pi}\right\|_{\Gamma_{0}(N)}}{\left\langle f_{\pi^{\prime}}, f_{\pi^{\prime}}\right\rangle_{R}}= & \frac{2^{2-k} D_{K}^{k-2} \pi(-1)^{m} \Gamma(k / 2+m) \Gamma(k / 2-m)}{\Gamma(k)} \cdot L\left(\frac{1}{2}, \pi_{K} \otimes \chi\right) \\
& \times \prod_{q \in S} \mathcal{P}\left(\varsigma_{q}^{(n)}, \varphi_{q}, \chi_{q}\right) \cdot \prod_{q \mid N^{-}} \zeta_{\mathbf{Q}_{q}}(1) \cdot \prod_{q \mid p N^{+}} \frac{\left\|\varphi_{\pi}\right\|_{q}}{\epsilon\left(\pi_{q}\right)} .
\end{aligned}
$$

We proceed to compute the local toric integrals $\mathcal{P}\left(\varsigma_{q}^{(n)}, \varphi_{q}, \chi_{q}\right)$ for $q \nmid p N^{+}$. At the archimedean place, $\pi_{\infty}^{\prime}=\rho_{k, \infty}$ and $\chi_{\infty}(t)=(t / \bar{t})^{m}$. In addition, $\tau_{\infty}^{N_{B}}=J$ and $\varphi_{\infty}=\mathbf{v}_{m}$ is characterized by $\rho_{k, \infty}(t)\left(\mathbf{v}_{m}\right)=$ $\chi_{\infty}(t)^{-1} \mathbf{v}_{m}$ for $t \in \mathbf{C}^{\times}$, so we have

Recall that

$$
\frac{\left\langle\rho_{k, \infty}(t) \mathbf{v}_{m}, \rho_{k, \infty}(J) \mathbf{v}_{m}\right\rangle_{k}}{\left\langle\mathbf{v}_{m}, \rho_{k, \infty}\left(\tau_{\infty}^{N_{B}}\right) \mathbf{v}_{m}\right\rangle_{k}} \cdot \chi_{\infty}(t)=1 \text { for all } t \in \mathbf{C}^{\times}=K_{\infty}^{\times} .
$$

$$
L\left(1, \operatorname{Ad} \pi_{\infty}\right)=2^{1-k} \pi^{-(k+1)} \Gamma(k) ; L\left(\frac{1}{2}, \pi_{K} \otimes \chi\right)=\Gamma_{\mathbf{C}}(k / 2+m) \Gamma_{\mathbf{C}}(k / 2-m) .
$$


We conclude that

$$
\mathcal{P}\left(1, \mathbf{v}_{m}, \chi_{\infty}\right)=\frac{\Gamma(k)}{2 \pi \Gamma(k / 2+m) \Gamma(k / 2-m)} \cdot \operatorname{vol}\left(\mathbf{C}^{\times} / \mathbf{R}^{\times}, d t_{\infty}\right)=\frac{\Gamma(k)}{\pi \Gamma(k / 2+m) \Gamma(k / 2-m)} .
$$

If $q \nmid p N$, then $\varphi_{q}$ is the spherical vector in $\pi_{q}$, and by Wal85, Lemme 14] (use the fact $i_{q}(J) \in i_{q}\left(K_{q}^{\times}\right) \mathrm{GL}_{2}\left(\mathbf{Z}_{q}\right)$ )

$$
\mathcal{P}\left(\varsigma_{q}, \varphi_{q}, \chi_{q}\right)=\left|D_{K}\right|_{q}^{\frac{1}{2}} .
$$

Suppose that $q \mid N^{-}$. Then $\pi_{q}^{\prime}=\xi \circ \mathrm{N}$ is the one dimensional representation of a unramified quadratic character $\xi: \mathbf{Q}_{q}^{\times} \rightarrow \mathbf{C}^{\times}$, and $\pi_{q}=\sigma\left(\xi|\cdot|^{\frac{1}{2}}, \xi|\cdot|^{-\frac{1}{2}}\right)$, and a simple calculation of local root numbers shows that

$$
\left(\chi_{q} \cdot \xi \circ \mathrm{N}\right)\left(\varpi_{K_{q}}\right)+\epsilon_{q}\left(\pi_{K}, \chi\right)=0 .
$$

In particular, if $K_{q} / \mathbf{Q}_{q}$ is unramified, then $\epsilon_{q}\left(\pi_{K}, \chi\right)=-1$. Therefore, we find that

$$
\mathcal{P}\left(1, \varphi_{q}, \chi_{q}\right)=\zeta_{\mathbf{Q}_{q}}(1)^{-1}\left|D_{K}\right|_{q}^{\frac{1}{2}} \cdot \begin{cases}1 & \cdots K_{q} / \mathbf{Q}_{q} \text { is unramified, } \\ \left(1-\epsilon_{q}\left(\pi_{K}, \chi\right)\right) & \cdots K_{q} / \mathbf{Q}_{q} \text { is ramified } .\end{cases}
$$

Combining (3.12), (3.13), (3.14) and (3.11), we obtain the desired formula.

3.6. Local toric integrals at $q \mid p N^{+}$. In this subsection, we carry out the computation of the local toric integral $\mathcal{P}\left(\varsigma_{q}^{(n)}, \varphi_{q}, \chi_{q}\right)$ using the Whittaker model for $q \mid p N^{+}$. Let $F=\mathbf{Q}_{q}$ and $E=K_{q}$ and write $\pi=\pi_{q}^{\prime} \simeq \pi_{q}, \chi=\chi_{q}$ and $\psi=\psi_{q}$ for brevity. Define the toric integral for Whittaker functions $W \in \mathcal{W}(\pi, \psi)$ by

$$
\boldsymbol{P}(W, \chi):=\frac{\left|D_{K}\right|^{-\frac{1}{2}} L\left(1, \tau_{E / F}\right)}{\zeta_{F}(1)} \cdot \int_{E^{\times} / F^{\times}} \mathbf{b}_{q}\left(\pi\left(t_{q}\right) W, \pi(J) W\right) \cdot \chi\left(t_{q}\right) d t_{q} .
$$

Lemma 3.7. Let $J_{q}^{(n)}:=\left(\varsigma_{q}^{(n)}\right)^{-1} J_{\varsigma_{q}}^{(n)}$. For $q \mid N^{+}$, we have

$$
\pi\left(J_{q}^{(n)}\right) \varphi_{q}=\epsilon(\pi) \pi\left(\left(\begin{array}{cc}
N^{+} & 0 \\
0 & 1
\end{array}\right)\right) \varphi_{q} .
$$

Proof. A straightforward computation shows that

$$
J_{q}^{(n)}=\sqrt{\beta} \cdot\left(\begin{array}{ll}
0 & 1 \\
1 & 0
\end{array}\right) \text { if } q \mid N^{+} .
$$

Thus, by Lemma 3.1 we have $\pi\left(J_{q}^{(n)}\right) \varphi_{q}=\pi\left(\left(\begin{array}{cc}N^{+} & 0 \\ 0 & 1\end{array}\right)\right) \pi\left(\boldsymbol{\tau}^{N}\right) \varphi_{q}=\epsilon(\pi) \pi\left(\left(\begin{array}{cc}N^{+} & 0 \\ 0 & 1\end{array}\right)\right) \varphi_{q}$.

Proposition 3.8. Let $q \mid N^{+}$. Write $q=\mathfrak{q} \overline{\mathfrak{q}}$ in $K$ with $\mathfrak{q} \mid \mathfrak{N}^{+}$. If $\chi$ is unramified, then we have

$$
\mathcal{P}\left(\varsigma_{q}, \varphi_{q}, \chi\right) \cdot \frac{\left\|\varphi_{\pi}\right\|_{q}}{\left|D_{K}\right|^{\frac{1}{2}} \epsilon(\pi)}=\chi_{q}\left(\mathfrak{N}^{+}\right) .
$$

Proof. Since $q \nmid p$ is split in $E$, we have $L\left(1, \tau_{E / F}\right)=\zeta_{F}(1),\left|D_{K}\right|=1$ and $\varsigma_{q}^{(n)}=\varsigma_{q}$. By definition (3.5),

$$
\mathcal{P}\left(\varsigma_{q}^{(n)}, \varphi_{q}, \chi\right)=\frac{1}{\left\|\varphi_{\pi}\right\|_{q}} \cdot \frac{1}{L\left(\frac{1}{2}, \pi_{E} \otimes \chi\right)} \cdot \boldsymbol{P}\left(\pi\left(\varsigma_{q}\right) W_{\pi}, \chi\right) .
$$

Write $\chi=\left(\chi_{\mathfrak{q}}, \chi_{\bar{q}}\right)$. A straightforward computation shows that

$$
\begin{aligned}
\boldsymbol{P}\left(\pi\left(\varsigma_{q}\right) W_{\pi}, \chi\right) & =\epsilon(\pi) \int_{F^{\times}} \int_{F^{\times}} W_{\pi}\left(\left(\begin{array}{cc}
a t_{1} & 0 \\
0 & 1
\end{array}\right)\right) W_{\pi}\left(\left(\begin{array}{cc}
-a N^{+} & 0 \\
0 & 1
\end{array}\right)\right) \chi_{\mathfrak{q}}\left(t_{1}\right) d^{\times} a d^{\times} t_{1} \quad \text { (by Lemma 3.7) } \\
& =\epsilon(\pi) \chi_{\mathfrak{q}}\left(N^{+}\right) \int_{F^{\times}} \int_{F^{\times}} W_{\pi}\left(\left(\begin{array}{rr}
t_{1} & 0 \\
0 & 1
\end{array}\right) W_{\pi}\left(\left(\begin{array}{cc}
a & 0 \\
0 & 1
\end{array}\right)\right) \chi_{\mathfrak{q}}\left(t_{1}\right) \chi_{\mathfrak{q}}^{-1}(a) d^{\times} a d^{\times} t_{1}\right. \\
& =\chi_{\mathfrak{q}}\left(N^{+}\right) \epsilon(\pi) \Psi\left(\frac{1}{2}, W_{\pi}, \chi_{\mathfrak{q}}\right) \Psi\left(\frac{1}{2}, W_{\pi}, \chi_{\mathfrak{q}}^{-1}\right) \\
& =\chi_{\mathfrak{q}}\left(N^{+}\right) \epsilon(\pi) \cdot L\left(\frac{1}{2}, \pi_{E} \otimes \chi\right) .
\end{aligned}
$$

The last equality follows from (1.1). 
We continue to compute the local toric integral at the place $q=p$. Recall that we assume $\pi=\pi_{p}$ is a unramified principal series $\pi\left(\mu_{p}, \nu_{p}\right)$ or a special representation $\sigma\left(\mu_{p}, \nu_{p}\right)$ with unramified character $\mu_{p}$ and $\mu_{p} \nu_{p}^{-1}=|\cdot|$. Let $\mathcal{I}_{p}$ be the Iwahori subgroup given by

$$
\mathcal{I}_{p}=\left\{g=\left(\begin{array}{ll}
a & b \\
c & d
\end{array}\right) \in \mathrm{GL}_{2}\left(\mathbf{Z}_{p}\right) \mid c \in p \mathbf{Z}_{p}\right\} .
$$

By the complete description of Kirillov models in [Jac72], the function $\mu_{p}|\cdot|^{\frac{1}{2}}(a) \mathbb{I}_{\mathbf{Z}_{p}}(a)$ lies in the Kirillov model $K(\pi, \psi)$, and hence there exists a unique Whittaker function $W_{p}^{\dagger} \in \mathcal{W}(\pi, \psi)$

$$
W_{p}^{\dagger}\left(\left(\begin{array}{cc}
a & 0 \\
0 & 1
\end{array}\right)\right)=\mu_{p}|\cdot|^{\frac{1}{2}}(a) \mathbb{I}_{\mathbf{Z}_{p}}(a) .
$$

One can verify that $W_{p}^{\dagger}$ is invariant by $\mathcal{I}_{p}$ and is an $U_{p}$-eigenfunction with eigenvalue $\alpha_{p}=\mu_{p}(p)|p|^{-\frac{1}{2}}$. If $\pi$ is unramified, then

$$
\begin{aligned}
W_{\pi}\left(\left(\begin{array}{cc}
a & 0 \\
0 & 1
\end{array}\right)\right) & =|a|^{\frac{1}{2}} \mathbb{I}_{\mathbf{Z}_{p}(a) \frac{\mu_{p}(p a)-\nu_{p}(p a)}{\mu_{p}(p)-\nu_{p}(p)}} \\
W_{p}^{\dagger}(g) & =W_{\pi}(g)-\frac{1}{\alpha_{p}} \cdot W_{\pi}\left(g\left(\begin{array}{ll}
1 & 0 \\
0 & p
\end{array}\right)\right) .
\end{aligned}
$$

If $\pi$ is special, then it is well known that

$$
W_{\pi}=W_{p}^{\dagger} \text { and } \pi\left(\left(\begin{array}{cc}
0 & 1 \\
-p & 0
\end{array}\right)\right) W_{\pi}=-\alpha_{p} W_{\pi}
$$

We have

$$
\mathcal{P}\left(\varsigma_{q}^{(n)}, \varphi_{q}, \chi\right)=\frac{\left|D_{K}\right|^{\frac{1}{2}}}{\left\|\varphi_{\pi}\right\|_{p}} \cdot \frac{1}{L\left(\frac{1}{2}, \pi_{E} \otimes \chi\right)} \cdot \boldsymbol{P}\left(\pi\left(\varsigma_{q}^{(n)}\right) W_{p}^{\dagger}, \chi\right) .
$$

Definition 3.9. Define the $p$-adic multiplier $e_{p}(\pi, \chi)$ by

$$
e_{p}(\pi, \chi)= \begin{cases}1 & \text { if } \chi_{p} \text { is ramified; } \\ \left(1-\alpha_{p}^{-1} \chi(\mathfrak{p})\right)\left(1-\alpha_{p}^{-1} \chi(\overline{\mathfrak{p}})\right) & \text { if } \chi_{p} \text { is unramified, } p=\mathfrak{p} \overline{\mathfrak{p}} \text { is split; } \\ 1-\alpha_{p}^{-2} & \text { if } \chi_{p} \text { is unramified, } p=\mathfrak{p} \text { is inert; } \\ 1-\alpha_{p}^{-1} \chi(\mathfrak{p}) & \text { if } \chi_{p} \text { is unramified, } p=\mathfrak{p}^{2} \text { is ramified }\end{cases}
$$

(cf. [BD96, §2.10]).

Proposition 3.10. Suppose that $\chi$ has conductor $p^{s}$. Let $n=\max \{1, s\}$. Then

$$
\frac{1}{L\left(\frac{1}{2}, \pi_{E} \otimes \chi\right)} \cdot \boldsymbol{P}\left(\pi\left(\varsigma_{p}^{(n)}\right) W_{p}^{\dagger}, \chi\right)=e_{p}(\pi, \chi)^{2-\operatorname{ord}_{p}(N)} \cdot L\left(1, \tau_{E / F}\right)^{2} \cdot \begin{cases}\alpha_{p}^{2} p^{-2} & \cdots s=0, \\ p^{-s} & \cdots s>0 .\end{cases}
$$

Therefore, by (3.16) we have

$$
\mathcal{P}\left(\varsigma_{p}^{(n)}, \varphi_{p}, \chi\right) \cdot \frac{\left\|\varphi_{\pi}\right\|_{p}}{\left|D_{K}\right|^{\frac{1}{2}}}=e_{p}(\pi, \chi)^{2-\operatorname{ord}_{p}(N)} \cdot L\left(1, \tau_{E / F}\right)^{2} \cdot \begin{cases}\alpha_{p}^{2} p^{-2} & \cdots s=0, \\ p^{-s} & \cdots s>0 .\end{cases}
$$

Proof. For $t \in E$, we put

$$
\iota_{\varsigma}^{(n)}(t):=\left(\varsigma_{p}^{(n)}\right)^{-1} \iota(t) \varsigma_{p}^{(n)} .
$$

It is easy to see that $\iota_{\varsigma}^{(n)}(J) \in \mathcal{I}_{p}$ if $n \geq 1$.

Suppose that $p=\mathfrak{p} \overline{\mathfrak{p}}$ is split in $E$. Recall that $\delta=\boldsymbol{\theta}-\overline{\boldsymbol{\theta}} \in \mathcal{O}_{K_{\mathfrak{p}}}^{\times}=\mathbf{Z}_{p}^{\times}$. A direct computation shows that

$$
\iota_{\varsigma}^{(n)}(t)=\left(\begin{array}{cc}
1 & \delta^{-1} p^{-n} \\
0 & 1
\end{array}\right)\left(\begin{array}{cc}
t & \\
0 & \bar{t}
\end{array}\right)\left(\begin{array}{cc}
1 & -\delta^{-1} p^{-n} \\
0 & 1
\end{array}\right) .
$$


We find that

$$
\begin{aligned}
& \boldsymbol{P}\left(\pi\left(\varsigma_{p}^{(n)}\right) W_{p}^{\dagger}, \chi\right) \\
= & \int_{F^{\times}} \int_{F^{\times}} W_{p}^{\dagger}\left(\left(\begin{array}{cc}
a x & 0 \\
0 & 1
\end{array}\right)\left(\begin{array}{cc}
1 & -\delta^{-1} p^{-n} \\
0 & 1
\end{array}\right)\right) W_{p}^{\dagger}\left(\left(\begin{array}{cc}
-a & 0 \\
0 & 1
\end{array}\right)\left(\begin{array}{cc}
1 & -\delta^{-1} p^{-n} \\
0 & 1
\end{array}\right)\right) \chi_{\mathfrak{p}}(x) d^{\times} a d^{\times} x \\
= & \int_{\mathbf{z}_{p}-\{0\}} \psi\left(-\delta^{-1} p^{-n} x\right) \mu_{p} \chi_{\mathfrak{p}}|\cdot|^{\frac{1}{2}}(x) d^{\times} x \cdot \int_{\mathbf{Z}_{p}-\{0\}} \psi\left(\delta^{-1} p^{-n} a\right) \mu_{p} \chi_{\mathfrak{p}}^{-1}|\cdot|^{\frac{1}{2}}(a) d^{\times} a .
\end{aligned}
$$

If $n=s \geq 1$, then $L\left(\frac{1}{2}, \pi_{E} \otimes \chi\right)=1$, and as

$$
\int_{\mathbf{Z}_{p}^{\times}} \psi\left(p^{-r} x\right) \chi_{\mathfrak{p}}^{ \pm}(x) d^{\times} x=0 \text { for all } r<n,
$$

we find that

$$
\begin{aligned}
\boldsymbol{P}\left(\pi\left(\varsigma_{p}^{(n)}\right) W_{p}^{\dagger}, \chi\right) & =\int_{\mathbf{z}_{p}^{\times}} \psi\left(-\delta^{-1} p^{-n} x\right) \chi_{\mathfrak{p}}(x) d^{\times} x \cdot \int_{\mathbf{Z}_{p}^{\times}} \psi\left(\delta^{-1} p^{-n} a\right) \chi_{\mathfrak{p}}^{-1}(a) d^{\times} a \\
& =\epsilon\left(1, \chi_{\mathfrak{p}}, \psi\right) \epsilon\left(1, \chi_{\mathfrak{p}}^{-1}, \psi\right) \cdot \zeta_{F}(1)^{2} \\
& =\left|p^{n}\right| L\left(1, \tau_{E / F}\right)^{2} .
\end{aligned}
$$

If $s=0$ and $n=1$, then $\chi$ is unramified and

$$
\begin{aligned}
\boldsymbol{P}\left(\pi\left(\varsigma_{p}^{(n)}\right) W_{p}^{\dagger}, \chi\right) & =\left(\frac{-|p|}{1-|p|}+\frac{\mu_{p} \chi_{\mathfrak{p}}|\cdot|^{\frac{1}{2}}(p)}{1-\mu_{p} \chi_{\mathfrak{p}}|\cdot|^{\frac{1}{2}}(p)}\right) \cdot\left(\frac{-|p|}{1-|p|}+\frac{\mu_{p} \chi_{\mathfrak{p}}^{-1}|\cdot|^{\frac{1}{2}}(p)}{1-\mu_{p} \chi_{\mathfrak{p}}^{-1}|\cdot|^{\frac{1}{2}}(p)}\right) \\
& =\frac{1-\mu_{p}^{-1} \chi_{\mathfrak{p}}^{-1}|\cdot|^{\frac{1}{2}}(p)}{1-\mu_{p} \chi_{\mathfrak{p}}|\cdot|^{\frac{1}{2}}(p)} \cdot \frac{1-\mu_{p}^{-1} \chi_{\mathfrak{p}}|\cdot|^{\frac{1}{2}}(p)}{1-\mu_{p} \chi_{\mathfrak{p}}^{-1}|\cdot|^{\frac{1}{2}}(p)} \cdot \zeta_{F}(1)^{2} \cdot \mu_{p}^{2}(p)|p| \\
& =\alpha_{p}^{2}|p|^{2} \cdot \frac{L\left(\frac{1}{2}, \mu_{p} \chi_{\mathfrak{p}}\right) L\left(\frac{1}{2}, \mu_{p} \chi_{\overline{\mathfrak{p}}}\right)}{L\left(\frac{1}{2}, \nu_{p} \chi_{\bar{p}}\right) L\left(\frac{1}{2}, \nu_{p} \chi_{\mathfrak{p}}\right)} \cdot \zeta_{F}(1)^{2} \quad\left(\nu_{p}=\mu_{p}^{-1}, \chi_{\overline{\mathfrak{p}}}=\chi_{\mathfrak{p}}^{-1}\right) \\
& =\alpha_{p}^{2}|p|^{2} \cdot L\left(\frac{1}{2}, \pi \otimes \chi\right) \cdot L\left(1, \tau_{E / F}\right)^{2} \cdot\left\{\begin{array}{ll}
\left(1-\alpha_{p}^{-1} \chi_{\mathfrak{p}}^{-1}(p)\right)^{2}\left(1-\alpha_{p}^{-1} \chi_{\overline{\mathfrak{p}}}^{-1}(p)\right)^{2} & \cdots p \nmid N, \\
\left(1-\alpha_{p}^{-1} \chi_{\mathfrak{p}}^{-1}(p)\right)\left(1-\alpha_{p}^{-1} \chi_{\overline{\mathfrak{p}}}^{-1}(p)\right) & \cdots p \mid N .
\end{array} .\right.
\end{aligned}
$$

This proves the formula in the split case.

Now we assume that $p$ is non-split. We introduce the matrix coefficient $\mathbf{m}^{\dagger}: G\left(\mathbf{Q}_{p}\right)=\mathrm{GL}_{2}\left(\mathbf{Q}_{p}\right) \rightarrow \mathbf{C}$ defined by

$$
\mathbf{m}^{\dagger}(g):=\mathbf{b}\left(\pi(g) W_{p}^{\dagger}, W_{p}^{\dagger}\right) .
$$

The function $\mathbf{m}^{\dagger}(g)$ only depends on the double coset $\mathcal{I}_{p} g \mathcal{I}_{p}$, and by definition

$$
\mathbf{m}^{\dagger}(1)=\frac{1}{1-\mu_{p}^{2}(p)|p|}
$$

Put

$$
\boldsymbol{P}^{*}:=\int_{E^{\times} / F^{\times}} \mathbf{m}^{\dagger}\left(\iota_{\varsigma}^{(n)}(t)\right) \chi(t) d t
$$

It is clear that

$$
\boldsymbol{P}\left(\pi\left(\varsigma_{p}^{(n)}\right) W_{p}^{\dagger}, \chi\right)=\boldsymbol{P}^{*} \cdot \frac{\left|D_{K}\right|^{-\frac{1}{2}} L\left(1, \tau_{E / F}\right)}{\zeta_{F}(1)} .
$$

To compute $\boldsymbol{P}^{*}$, we make some observations. Let $r \in \mathbf{Z}_{\geq 0}$. For $y \in p^{r} \mathbf{Z}_{p}^{\times}$, we have

$$
\iota_{\varsigma}^{(n)}(1+y \boldsymbol{\theta}) \in \mathcal{I}_{p} \mathbf{w}\left(\begin{array}{cc}
p^{n-r} & 0 \\
0 & p^{r-n}
\end{array}\right) \mathcal{I}_{p} \text { if } 0 \leq r<n \quad\left(\mathbf{w}=\left(\begin{array}{cc}
0 & 1 \\
-1 & 0
\end{array}\right)\right)
$$

and $\iota_{\varsigma}^{(n)}(1+y \boldsymbol{\theta}) \in \mathcal{I}_{p}$ if $r \geq n$. For $y \in p \mathbf{Z}_{p}$, we have

$$
\iota_{\varsigma}^{(n)}(y+\boldsymbol{\theta}) \in \mathcal{I}_{p} \mathbf{w}\left(\begin{array}{cc}
p^{n+e-1} & 0 \\
0 & p^{-n}
\end{array}\right) \mathcal{I}_{p},
$$


where $e=1$ if $p$ is inert and $e=2$ if $p$ is ramified. Put

$$
X_{r}:=\int_{p^{r} \mathbf{Z}_{p}} \chi(1+y \boldsymbol{\theta}) d^{\prime} y,
$$

where $d^{\prime} y$ is the Haar measure on $\mathbf{Z}_{p}$ such that $\operatorname{vol}\left(\mathbf{Z}_{p}, d^{\prime} y\right)=L\left(1, \tau_{E / F}\right)\left|D_{K}\right|^{\frac{1}{2}}$. Using the decomposition

$$
E^{\times}=F^{\times}\left(1+\mathbf{Z}_{p} \boldsymbol{\theta}\right) \bigsqcup F^{\times}\left(p \mathbf{Z}_{p}+\boldsymbol{\theta}\right),
$$

we find that

$$
\begin{aligned}
\boldsymbol{P}^{*} & =\int_{\mathbf{Z}_{p}} \chi(1+y \boldsymbol{\theta}) \mathbf{m}^{\dagger}\left(\iota_{\varsigma}^{(n)}(1+y \boldsymbol{\theta})\right) d^{\prime} y+\int_{p \mathbf{Z}_{p}} \chi(y+\boldsymbol{\theta}) \mathbf{m}^{\dagger}\left(\iota_{\varsigma}^{(n)}(y+\boldsymbol{\theta})\right)|y+\boldsymbol{\theta}|_{E}^{-1} d^{\prime} y \\
& =X_{n} \cdot \mathbf{m}^{\dagger}(1)+\sum_{r=0}^{n-1}\left(X_{r}-X_{r+1}\right) \mathbf{m}^{0}\left(\mathbf{w}\left(\begin{array}{cc}
p^{n-r} & 0 \\
0 & p^{r-n}
\end{array}\right)\right)+Y_{0} \cdot \mathbf{m}^{\dagger}\left(\mathbf{w}\left(\begin{array}{cc}
p^{n+e-1} & 0 \\
0 & p^{-n}
\end{array}\right)\right),
\end{aligned}
$$

where

$$
Y_{0}:=\int_{p \mathbf{Z}_{p}} \chi(y+\boldsymbol{\theta}) d^{\prime} y \cdot|\varpi|^{1-e} .
$$

Suppose that $n=s \geq 1$. Then it is easy to verify

- $X_{r}=0$ if $0<r<n$,

- $X_{0}+Y_{0}=0$ if $p$ is inert, and $X_{0}=Y_{0}=0$ if $p$ is ramified.

It follows from (3.18) that

$$
\boldsymbol{P}^{*}=X_{n} \cdot \mathbf{m}^{\dagger}(1)+\left(-X_{n}\right) \cdot \mathbf{m}^{\dagger}\left(\mathbf{w}\left(\begin{array}{cc}
p & 0 \\
0 & p^{-1}
\end{array}\right)\right) \quad\left(X_{n}=\left|p^{n}\right| L\left(1, \tau_{E / F}\right)\left|D_{K}\right|^{\frac{1}{2}}\right) .
$$

If $\pi$ is a unramified principal series, then

$$
\begin{aligned}
\boldsymbol{P}^{*} & =\mathbf{b}\left(W_{\pi}-\pi\left(\left(\begin{array}{cc}
p^{-1} & 0 \\
0 & p
\end{array}\right)\right) W_{\pi}, W_{p}^{\dagger}\right) \cdot X_{n} \\
& =\left(\frac{\mu_{p}(p)}{1-\mu_{p} \nu_{p}^{-1}|\cdot|(p)}-\frac{\nu_{p}(p)}{1-|p|}\right) \cdot \frac{1-\mu_{p} \nu_{p}^{-1}|\cdot|(p)}{\mu_{p}(p)-\nu_{p}(p)} \cdot X_{n} \\
& =\zeta_{F}(1) \cdot\left|p^{n}\right| L\left(1, \tau_{E / F}\right)\left|D_{K}\right|^{\frac{1}{2}} .
\end{aligned}
$$

If $\pi$ is special, then by (3.15),

$$
\begin{aligned}
\boldsymbol{P}^{*} & =\frac{1+\mu_{p} \nu_{p}^{-1}(p)}{1-\mu_{p} \nu_{p}^{-1}|\cdot|(p)} \cdot X_{n} \\
& =\zeta_{F}(1) \cdot\left|p^{n}\right| L\left(1, \tau_{E / F}\right)\left|D_{K}\right|^{\frac{1}{2}} \quad\left(\mu_{p} \nu_{p}^{-1}(p)=|p|\right) .
\end{aligned}
$$

Suppose that $s=0$ and $n=1$. Then we have

$$
\boldsymbol{P}^{*}=X_{1} \cdot \mathbf{m}^{\dagger}(1)+\left(X_{0}-X_{1}\right) \cdot \mathbf{m}^{\dagger}\left(\mathbf{w}\left(\begin{array}{cc}
p & 0 \\
0 & p^{-1}
\end{array}\right)\right)+Y_{0} \cdot \mathbf{m}^{\dagger}\left(\mathbf{w}\left(\begin{array}{cc}
p^{e} & 0 \\
0 & p^{-1}
\end{array}\right)\right) .
$$

- If $p$ is inert, then $X_{0}=1-X_{1}=L\left(1, \tau_{E / F}\right)$ and $Y_{0}=X_{1}$.

- If $p$ is ramified, then $X_{0}=\left|D_{K}\right|^{\frac{1}{2}}$ and $Y_{0}=\chi\left(\varpi_{E}\right)\left|D_{K}\right|^{\frac{1}{2}}$.

Case (1): $p$ is inert and $\pi$ is unramified. Then

$$
\begin{aligned}
\boldsymbol{P}^{*} & =\mathbf{m}^{\dagger}(1)+X_{0} \cdot\left(\mathbf{m}^{\dagger}\left(\mathbf{w}\left(\begin{array}{cc}
p & 0 \\
0 & p^{-1}
\end{array}\right)\right)-\mathbf{m}^{\dagger}(1)\right) \\
& =\frac{1}{1-\mu_{p}^{2}(p)|p|}-\frac{1}{1-|p|^{2}} \\
& =\alpha_{p}^{2}|p|^{2}\left(1-\alpha_{p}^{-2}\right)^{2} \cdot \zeta_{F}(1) L\left(1, \tau_{E / F}\right) \cdot L\left(\frac{1}{2}, \pi_{E} \otimes \chi\right)
\end{aligned}
$$


Case (2): $p$ is inert and $\pi$ is special. Then $\alpha_{p}^{2}=1$, so $e_{p}(\pi, \chi)=0$ and

$$
\boldsymbol{P}^{*}=\frac{|p|-\alpha_{p} \mu_{p}(p)|p|^{\frac{1}{2}}}{1-|p|^{2}}=0 .
$$

Case (3): $p$ is ramified and $\pi$ is unramified. Note that

$$
\mathbf{m}^{\dagger}\left(\mathbf{w}\left(\begin{array}{cc}
p^{2} & 0 \\
0 & p^{-1}
\end{array}\right)\right)=\mu|\cdot|^{\frac{1}{2}}(p) \mathbf{m}^{\dagger}\left(\mathbf{w}\left(\begin{array}{cc}
p & 0 \\
0 & p^{-1}
\end{array}\right)\right) .
$$

Let $\beta:=\chi\left(\varpi_{E}\right) \mu_{p}|\cdot|^{\frac{1}{2}}(p)$. Then we have

$$
\begin{aligned}
\left|D_{K}\right|^{-\frac{1}{2}} \boldsymbol{P}^{*} & =|p| \cdot \mathbf{m}^{\dagger}(1)+(1-|p|) \cdot \mathbf{m}^{\dagger}\left(\mathbf{w}\left(\begin{array}{cc}
p & 0 \\
0 & p^{-1}
\end{array}\right)\right)+\chi\left(\varpi_{E}\right) \mathbf{m}^{\dagger}\left(\mathbf{w}\left(\begin{array}{cc}
p^{2} & 0 \\
0 & p^{-1}
\end{array}\right)\right) \\
& =(1-|p|+\beta)\left(\mathbf{m}^{\dagger}\left(\mathbf{w}\left(\begin{array}{cc}
p & 0 \\
0 & p^{-1}
\end{array}\right)\right)-\mathbf{m}^{\dagger}(1)\right)+(1+\beta) \mathbf{m}^{\dagger}(1) \\
& =-\frac{(1-|p|+\beta)}{1-|p|}+\frac{1+\beta}{1-\beta^{2}} \quad\left(\mu_{p}^{2}(p)|p|=\beta^{2}=\alpha_{p}^{2}|p|^{2}\right) \\
& =\alpha_{p}^{2}|p|^{2} \cdot\left(1-\chi\left(\varpi_{E}\right) \alpha_{p}^{-1}\right)^{2} \cdot \zeta_{F}(1) L\left(\frac{1}{2}, \pi_{E} \otimes \chi\right) .
\end{aligned}
$$

Case (4): $p$ is ramified and $\pi$ is special. Then $\alpha_{p}^{2}=\chi\left(\varpi_{E}\right)^{2}=1$ and

$$
\begin{aligned}
\left|D_{K}\right|^{-\frac{1}{2}} \boldsymbol{P}^{*} & =|p| \mathbf{m}^{\dagger}(1)+(1-|p|)\left(-\alpha_{p}\right) \mathbf{m}^{\dagger}\left(\left(\begin{array}{cc}
p^{-1} & 0 \\
0 & 1
\end{array}\right)\right)+\chi\left(\varpi_{E}\right)\left(-\alpha_{p}\right) \mathbf{m}^{\dagger}\left(\left(\begin{array}{cc}
p^{-2} & 0 \\
0 & 1
\end{array}\right)\right) \\
& =\frac{|p|^{2}\left(1-\chi\left(\varpi_{E}\right) \alpha_{p}\right)}{1-\mu^{2}(p)|p|} .
\end{aligned}
$$

We find that if $\chi\left(\varpi_{E}\right)=\alpha_{p}$, then $\boldsymbol{P}^{*}=e_{p}(\pi, \chi)=0$ and if $\chi\left(\varpi_{E}\right)=-\alpha_{p}$, then

$$
\begin{aligned}
\left|D_{K}\right|^{-\frac{1}{2}} \boldsymbol{P}^{*} & =\frac{2|p|^{2}}{\left(1-\chi\left(\varpi_{E}\right) \mu_{p}|\cdot|^{\frac{1}{2}}(p)\right)\left(1+\chi\left(\varpi_{E}\right) \mu_{p}|\cdot|^{\frac{1}{2}}(p)\right)} \\
& =|p|^{2}\left(1-\alpha_{p}^{-1} \chi\left(\varpi_{E}\right)\right) \cdot \zeta_{F}(1) L\left(\frac{1}{2}, \pi_{E} \otimes \chi\right) .
\end{aligned}
$$

The above calculations together with (3.17) completes the proof in the inert or ramified case.

3.7. Central value formula. We are ready to prove the central value formula connecting the toric period integral of the $p$-stabilized form $\varphi_{\pi^{\prime}}^{\dagger}$ in (3.2) and the central $L$-value of $\pi_{K}$ twisted by anticyclotomic characters $\chi$ satisfying (crit).

Theorem 3.11. Suppose that $\chi$ has conductor $p^{s}$. Let $n=\max \{1, s\}$. Then we have

$$
\begin{aligned}
P\left(\varsigma^{(n)}, \varphi_{\pi^{\prime}}^{\dagger}, \chi\right)^{2} \cdot \frac{\left\|\varphi_{\pi}\right\|_{\Gamma_{0}(N)}}{\left\langle f_{\pi^{\prime}}, f_{\pi^{\prime}}\right\rangle_{R}}= & \frac{2^{2-k}(-1)^{m} D_{K}^{k-2}}{\sqrt{D_{K}}} \cdot L\left(\frac{1}{2}, \pi_{K} \otimes \chi\right) \cdot e_{p}(\pi, \chi)^{2-\operatorname{ord}_{p}(N)} \cdot L\left(1, \tau_{K_{p} / \mathbf{Q}_{p}}\right)^{2} \\
& \times \chi\left(\mathfrak{N}^{+}\right) \epsilon\left(\pi_{p}\right) \prod_{q \mid\left(D_{K}, N^{-}\right)}\left(1-\epsilon_{q}\left(\pi_{K}, \chi\right)\right) \cdot \begin{cases}\alpha_{p}^{2} p^{-2} & \cdots s=0 \\
p^{-s} & \cdots s>0 .\end{cases}
\end{aligned}
$$

Proof. This follows from Corollary 3.6, Proposition 3.8 and Proposition 3.10 .

\section{Theta Elements And $p$-ADiC $L$-FunCtions}

4.1. $\ell$-adic modular forms. Let $\ell \nmid N^{-}$be a rational prime in 2.1 . We briefly review $\ell$-adic modular forms on $B^{\times}$. Let $A$ be a $\mathcal{O}_{K_{\mathrm{r}}}$-algebra. For an open compact subgroup $U \subset \widehat{R}^{\times}$, we define the space of $\ell$-adic modular forms of weight $k$ and level $U$ by

$$
\mathcal{M}_{k}(U, A)=\left\{\widehat{f}: \widehat{B}^{\times} \rightarrow L_{k}(A) \mid \widehat{f}(\alpha g u)=\rho_{k}\left(u_{\ell}^{-1}\right) \widehat{f}(g), \alpha \in B^{\times}, u \in U \widehat{\mathbf{Q}}^{\times}\right\} .
$$


We write $\mathcal{M}_{k}\left(N_{B}, A\right):=\mathcal{M}_{k}\left(\widehat{R}^{\times}, A\right)$. Recall that we have fixed an embedding $\iota_{\ell}: \overline{\mathbf{Q}} \hookrightarrow \mathbf{C}_{\ell}$. Let $\lambda$ and $\mathfrak{l}$ be the primes of $\overline{\mathbf{Q}}$ and $K$ induced by $\iota_{\ell}$ respectively. We let $i_{K_{\mathfrak{l}}}: B \hookrightarrow M_{2}\left(K_{\mathfrak{l}}\right)$ be the composition $i_{K_{\mathfrak{l}}}:=\iota_{\ell} \circ i_{K}$ defined in (2.3). Define $\rho_{k, \ell}: B_{\ell}^{\times} \rightarrow$ Aut $L_{k}\left(\mathbf{C}_{\ell}\right)$ by

$$
\rho_{k, \ell}(g):=\rho_{k}\left(i_{K_{\mathfrak{l}}}(g)\right) \text {. }
$$

By definition, $\rho_{k, \ell}$ is compatible with $\rho_{k, \infty}$ in the sense that $\rho_{k, \ell}(g)=\rho_{k, \infty}(g)$ for every $g \in B^{\times}$, and one checks that

$$
\rho_{k, \ell}(g)=\rho_{k}\left(\gamma_{\mathfrak{l}} i_{\ell}(g) \gamma_{\mathfrak{l}}^{-1}\right), \gamma_{\mathfrak{l}}:=\left(\begin{array}{cc}
\sqrt{\beta} & -\sqrt{\beta} \overline{\boldsymbol{\theta}} \\
-1 & \boldsymbol{\theta}
\end{array}\right) \in \mathrm{GL}_{2}\left(K_{\mathfrak{l}}\right) .
$$

Here $i_{\ell}: B_{\ell} \simeq M_{2}\left(\mathbf{Q}_{\ell}\right)$ is the isomorphism fixed in (2.1). If $A=A\left[\frac{1}{\ell}\right]$, there is an isomorphism:

$$
\mathbf{M}_{k}\left(N_{B}, A\right) \stackrel{\sim}{\rightarrow} \mathcal{M}_{k}\left(N_{B}, A\right), f \mapsto \widehat{f}(g):=\rho_{k}\left(\gamma_{\mathfrak{l}}^{-1}\right) \rho_{k, \ell}\left(g_{\ell}^{-1}\right) f(g) .
$$

We call $\widehat{f}$ the $\ell$-adic avatar of $f \in \mathbf{M}_{k}\left(N_{B}, A\right)$.

Let $\mathbf{Q}(\pi)$ be the Hecke field of $\pi$. In other words, $\mathbf{Q}(\pi)$ is the finite extension of $\mathbf{Q}$ generated by Fourier coefficients of the elliptic new form $f_{\pi}$. Let $\mathcal{O}_{\pi, \ell} \subset \mathbf{C}_{\ell}$ be the completion of the ring of integers of $\mathbf{Q}(\pi)$ with respect to $\lambda$. The $\mathcal{O}_{\pi, \ell}$-module $\mathcal{M}_{k}\left(N_{B}, \mathcal{O}_{\pi, \ell}\right)\left[\pi_{f}^{\prime}\right]:=\mathcal{M}_{k}\left(N_{B}, \mathcal{O}_{\pi, \ell}\right) \cap \mathcal{M}_{k}\left(N_{B}, \mathbf{C}_{\ell}\right)\left[\pi_{f}^{\prime}\right]$ has rank one. We say $f_{\pi^{\prime}} \in \mathbf{M}_{k}\left(N_{B}, \mathbf{C}\right)\left[\pi_{f}^{\prime}\right]$ is $\lambda$-adically normalized if its $\ell$-adic avatar $\widehat{f}_{\pi^{\prime}}$ is a generator of $\mathcal{M}_{k}\left(N_{B}, \mathcal{O}_{\pi, \ell}\right)\left[\pi_{f}^{\prime}\right]$ over $\mathcal{O}_{\pi, \ell}$. This is equivalent to the following condition:

$$
\widehat{f}_{\pi^{\prime}}\left(g_{0}\right) \not \equiv 0(\bmod \lambda) \text { for some } g_{0} \in G\left(\mathbf{A}_{f}\right) \text {. }
$$

4.2. Theta elements. Let $n \geq 1$ be a positive integer. Let $\mathcal{G}_{n}=K^{\times} \backslash \widehat{K}^{\times} / \widehat{\mathcal{O}}_{n}^{\times}$be the Picard group of the order $\mathcal{O}_{n}$. We identify $\mathcal{G}_{n}$ with the Galois group of the ring class field of conductor $p^{n}$ over $K$ via geometrically normalized reciprocity law. Denote by $[\cdot]_{n}: \widehat{K}^{\times} \rightarrow \mathcal{G}_{n}, a \mapsto[a]_{n}$ the natural projection map. We consider the automorphic form $\varphi_{\pi^{\prime}}^{[m]}=\Psi\left(\mathbf{v}_{m}^{*} \otimes f_{\pi^{\prime}}\right)$ in (3.1) and define the function

$$
\widehat{\varphi}_{\pi^{\prime}}^{[m]}: \widehat{K}^{\times} \rightarrow \mathbf{C}, \quad a \mapsto \widehat{\varphi}_{\pi^{\prime}}^{[m]}(a):=\varphi_{\pi^{\prime}}^{[m]}\left(x_{n}(a)\right) \iota_{p}^{-1}\left(\overline{a_{p}} / a_{p}\right)^{m} .
$$

Replacing $f_{\pi^{\prime}}$ by $f_{\pi^{\prime}}^{\dagger}$, we can define the $p$-stabilizations $\left(\varphi_{\pi^{\prime}}^{[m]}\right)^{\dagger}:=\Psi\left(\mathbf{v}_{m}^{*} \otimes f_{\pi^{\prime}}^{\dagger}\right)$ and $\left(\widehat{\varphi}_{\pi^{\prime}}^{[m]}\right)^{\dagger}$ in a similar manner. By (3.3), we can verify that $\widehat{\varphi}_{\pi^{\prime}}^{[m]}$ factors through $K^{\times} \backslash \widehat{K}^{\times}$.

Definition 4.1. Fix a set $\Xi_{n}$ of representatives of $\mathcal{G}_{n}$ in $K^{\times} \backslash \widehat{K}^{\times}$, define the $n$-th theta element $\Theta_{n}^{[m]}\left(f_{\pi^{\prime}}^{\dagger}\right) \in$ $\mathbf{C}\left[\mathcal{G}_{n}\right]$ of weight $m$ is defined by

$$
\begin{aligned}
\Theta_{n}^{[m]}\left(f_{\pi^{\prime}}^{\dagger}\right) & :=\alpha_{p}^{-n} \cdot \sum_{a \in \Xi_{n}}\left(\widehat{\varphi}_{\pi^{\prime}}^{[m]}\right)^{\dagger}(a) \cdot[a]_{n} \\
& =\alpha_{p}^{-n} \cdot \sum_{a \in \Xi_{n}}\left(\varphi_{\pi^{\prime}}^{[m]}\right)^{\dagger}\left(x_{n}(a)\right) \iota_{p}^{-1}\left(\overline{a_{p}} / a_{p}\right)^{m} \cdot[a]_{n} .
\end{aligned}
$$

We consider theta elements of weight zero. The function $\widehat{\varphi}_{\pi^{\prime}}^{[0]}$ factors through $\mathcal{G}_{n}$, so we can extend $\widehat{\varphi}_{\pi^{\prime}}^{[0]}$ linearly to be a function $\widehat{\varphi}_{\pi^{\prime}}^{[0]}: \mathbf{C}\left[\mathcal{G}_{n}\right] \rightarrow \mathbf{C}$, and the definition of $\Theta_{n}^{[0]}\left(f_{\pi^{\prime}}^{\dagger}\right)$ does not depend on the choice of $\Xi_{n}$. Let $P_{n}:=[1]_{n} \in \mathcal{G}_{n}$ be the distinguished Gross point of conductor $p^{n}$. Then

$$
\widehat{\varphi}_{\pi^{\prime}}^{[0]}\left(\sigma\left(P_{n}\right)\right)=\varphi_{\pi^{\prime}}^{[0]}\left(x_{n}(a)\right) \text { if } \sigma=[a]_{n} \in \mathcal{G}_{n} .
$$

Define the regularized Gross point $P_{n}^{\dagger}$ as follows. If $p \nmid N$, we define $P_{n}^{\dagger}$ by the formal sum

$$
P_{n}^{\dagger}:=\frac{1}{\alpha_{p}^{n}} \cdot P_{n}-\frac{1}{\alpha_{p}^{n+1}} \cdot P_{n-1},
$$

and if $p \mid N$, we define $P_{n}^{\dagger}=\alpha_{p}^{-n} \cdot P_{n}$. We have

$$
\Theta_{n}^{[0]}\left(f_{\pi^{\prime}}^{\dagger}\right)=\sum_{\sigma \in \mathcal{G}_{n}} \widehat{\varphi}_{\pi^{\prime}}^{[0]}\left(\sigma\left(P_{n}^{\dagger}\right)\right) \cdot \sigma
$$

and $\left\{\Theta_{n}^{[0]}\left(f_{\pi^{\prime}}^{\dagger}\right)\right\}_{n}$ satisfy the following compatible relation. 
Lemma 4.2. Let $\pi_{n+1, n}: \mathcal{G}_{n+1} \rightarrow \mathcal{G}_{n}$ be the natural quotient map. We have

$$
\pi_{n+1, n}\left(\Theta_{n+1}^{[0]}\left(f_{\pi^{\prime}}^{\dagger}\right)\right)=\Theta_{n}^{[0]}\left(f_{\pi^{\prime}}^{\dagger}\right) .
$$

Proof. This is standard. For $n^{\prime}>n$, let $K_{n^{\prime}, n}:=\operatorname{Ker}\left(\mathcal{G}_{n^{\prime}} \rightarrow \mathcal{G}_{n}\right)$. Using the description

$$
K_{n^{\prime}, n}=\left[\left(\mathcal{O}_{n} \otimes \mathbf{Z}_{p}\right)^{\times}\right]_{n^{\prime}}=\left\{\left[1+p^{n} u \boldsymbol{\theta}\right]_{n^{\prime}} \mid u \in \mathbf{Z} / p^{n^{\prime}-n} \mathbf{Z}\right\},
$$

we find that

$$
\sum_{u \in K_{n+1, n}}\left(\varphi_{\pi^{\prime}}^{[0]}\right)^{\dagger}\left(x_{n+1}(a u)\right)=\left(\varphi_{\pi^{\prime}}^{[0]}\right)^{\dagger} \mid U_{p}\left(x_{n}(a)\right)=\alpha_{p} \cdot\left(\varphi_{\pi^{\prime}}^{[0]}\right)^{\dagger}\left(x_{n}(a)\right) .
$$

The lemma follows.

Let $f_{\pi} \in S_{k}\left(\Gamma_{0}(N)\right)$ be the elliptic new form corresponding to $\varphi_{\pi}$. The Fourier coefficients of the $q$-expansion $f_{\pi}(q)=\sum_{n>0} \mathbf{c}_{n}\left(f_{\pi}\right) q^{n}$ at the infinity cusp are given by

$$
\mathbf{c}_{n}\left(f_{\pi}\right)=W_{\pi, f}\left(\left(\begin{array}{ll}
n & 0 \\
0 & 1
\end{array}\right)\right) n^{\frac{k}{2}} \quad\left(W_{\pi, f}=\prod_{q<\infty} W_{\pi_{q}}\right) .
$$

Let $A_{p}:=p^{\frac{k}{2}-1} \alpha_{p}$ be a root of the Hecke polynomial of $f_{\pi}$ at $p$ i.e. $X^{2}-\mathbf{c}_{p}\left(f_{\pi}\right) X+p^{k-1}$ if $p \nmid N ; X-\mathbf{c}_{p}\left(f_{\pi}\right)$ if $p \mid N$. Let $\chi$ be an anticyclotomic Hecke character of conductor $p^{s}$ and weight $(m,-m)$ for an integer $-k / 2<$ $m<k / 2$. Recall that $\widehat{\chi}: K^{\times} \backslash \widehat{K}^{\times} \rightarrow \mathcal{O}_{\mathbf{C}_{p}}^{\times}$denotes the $p$-adic avatar of $\chi$ defined by $\widehat{\chi}(a)=\chi(a)\left(a_{p} / \overline{a_{p}}\right)^{m}$. We are going to give the interpolation formula of the square of

$$
\begin{aligned}
\widehat{\chi}\left(\Theta_{n}^{[m]}\left(f_{\pi^{\prime}}^{\dagger}\right)\right) & :=\alpha_{p}^{-n} \cdot \sum_{a \in \Xi_{n}}\left(\varphi_{\pi^{\prime}}^{[m]}\right)^{\dagger}\left(x_{n}(a)\right) \iota_{p}^{-1}\left(\left(\overline{a_{p}} / a_{p}\right)^{m} \cdot \widehat{\chi}(a)\right) \\
& =\alpha_{p}^{-n} \cdot \sum_{[a]_{n} \in \mathcal{G}_{n}}\left(\varphi_{\pi^{\prime}}^{[m]}\right)^{\dagger}\left(x_{n}(a)\right) \chi(a)
\end{aligned}
$$

for every $n \geq \max \{1, s\}$ in terms of the central value of the Rankin-Selberg $L$-function $L\left(f_{\pi} / K, \chi, s\right)$ attached to $f_{\pi}$ and the theta series attached to $\chi$. Recall that connection between the automorphic $L$-function $L\left(s, \pi_{K} \otimes\right.$ $\chi)$ and the Rankin $L$-series $L\left(f_{\pi} / K, \chi, s\right)$ is given by

$$
L\left(s, \pi_{K} \otimes \chi\right)=\Gamma_{\mathbf{C}}\left(s+\frac{k-1}{2}+m\right) \Gamma_{\mathbf{C}}\left(s+\frac{k-1}{2}-m\right) \cdot L\left(f_{\pi} / K, \chi, s+\frac{k-1}{2}\right) .
$$

Define the period $\Omega_{\pi, N^{-}}$of $\pi^{\prime}$ by

$$
\Omega_{\pi, N^{-}}:=\frac{(4 \pi)^{k}\left\|\varphi_{\pi}\right\|_{\Gamma_{0}(N)}}{\left\langle f_{\pi^{\prime}}, f_{\pi^{\prime}}\right\rangle_{R}}
$$

Proposition 4.3. Suppose that $\chi$ has the conductor of $p^{s}$. For every $n \geq \max \{s, 1\}$, we have the interpolation formula

$$
\begin{aligned}
\widehat{\chi}\left(\Theta_{n}^{[m]}\left(f_{\pi^{\prime}}^{\dagger}\right)^{2}\right)= & \Gamma\left(\frac{k}{2}+m\right) \Gamma\left(\frac{k}{2}-m\right) \cdot \frac{L\left(f_{\pi} / K, \chi, \frac{k}{2}\right)}{\Omega_{\pi, N^{-}}} \cdot e_{p}\left(\pi, \chi_{t} \nu\right)^{2-\operatorname{ord}_{p}(N)} \cdot A_{p}^{-2 s}\left(p^{s} D_{K}\right)^{k-1} \\
& \times \frac{u_{K}^{2}}{\sqrt{D_{K}}} \cdot \chi\left(\mathfrak{N}^{+}\right) \epsilon\left(\pi_{p}\right)(-1)^{m} \prod_{q \mid\left(D_{K}, N^{-}\right), q=\mathfrak{q}^{2}}\left(1-\epsilon\left(\pi_{q}\right) \chi(\mathfrak{q})\right) .
\end{aligned}
$$

Proof. We may assume $n=\max \{1, s\}$, using the argument in Lemma 4.2. By (3.3) and the definition of theta elements, we have

$$
P\left(\pi^{\prime}\left(\varsigma^{(n)}\right), \varphi_{\pi^{\prime}}^{\dagger}, \chi\right)=\operatorname{vol}\left(\widehat{\mathcal{O}}_{n}^{\times}\right) \alpha_{p}^{n} \cdot \widehat{\chi}\left(\Theta_{n}^{[m]}\left(f_{\pi^{\prime}}^{\dagger}\right)\right) \quad\left(\varphi_{\pi^{\prime}}^{\dagger}=\left(\varphi_{\pi^{\prime}}^{[m]}\right)^{\dagger}\right),
$$

where $\operatorname{vol}\left(\widehat{\mathcal{O}}_{n}^{\times}\right)$denotes the volume of the image of $\mathbf{C}^{\times} \widehat{\mathcal{O}}_{n}^{\times}$in $K^{\times} \mathbf{A}^{\times} \backslash \mathbf{A}_{K}^{\times}$with respect to the measure $d t$. Recall that $d t$ is chosen so that $\operatorname{vol}\left(K^{\times} \mathbf{A}^{\times} \backslash \mathbf{A}_{K}^{\times}, d t\right)=2 L\left(1, \tau_{K / \mathbf{Q}}\right)$. Using the class number formula, we have

$$
\operatorname{vol}\left(\widehat{\mathcal{O}}_{n}^{\times}\right)=\operatorname{vol}\left(\widehat{\mathcal{O}}_{K}^{\times}\right) \cdot L\left(1, \tau_{K_{p} / \mathbf{Q}_{p}}\right)|p|^{n}=\frac{4}{\sqrt{D_{K}} \cdot u_{K}} L\left(1, \tau_{K_{p} / \mathbf{Q}_{p}}\right) p^{-n} .
$$


Combining these equations, we find that

$$
\widehat{\chi}\left(\Theta_{n}^{[m]}\left(f_{\pi^{\prime}}^{\dagger}\right)\right)=\frac{\sqrt{D_{K}} \cdot u_{K}}{4} \cdot \frac{\alpha_{p}^{-n} p^{n}}{L\left(1, \tau_{K_{p} / \mathbf{Q}_{p}}\right)} \cdot P\left(\varsigma^{(n)}, \varphi_{\pi^{\prime}}^{\dagger}, \chi\right) .
$$

Thus, the proposition follows from Theorem 3.11, (4.2) and the formula:

$$
\epsilon_{q}\left(\pi_{K}, \chi\right)=\epsilon\left(\pi_{q}\right) \chi(\mathfrak{q}) \text { for } q \mid\left(D_{K}, N^{-}\right), q=\mathfrak{q}^{2} .
$$

Remark. This proposition verifies [BDIS02, Conjecture 2.17], and hence removes the assumption in Theorem 3.4 loc.cit.

4.3. $p$-adic $L$-functions. We shall use theta elements to construct anticyclotomic $p$-adic $L$-functions attached to $f$ and derive the evaluation formulae. We begin with a key observation.

Lemma 4.4. Let $A \subset \mathbf{C}_{p}$ be an $\mathcal{O}_{K_{\mathfrak{p}}}$-algebra. Let $f \in \mathbf{M}_{k}\left(N_{B}, \mathbf{C}_{p}\right)$ such that the p-adic avatar $\widehat{f} \in$ $\mathcal{M}_{k}\left(N_{B}, A\right)$ is a p-adic modular form over $A$. Let

$$
f_{\mathbf{v}_{m}}:=\Psi\left(\mathbf{v}_{m}^{*} \otimes f\right) \in \mathcal{A}(G)
$$

be defined as in (2.11). For $a \in \widehat{K}^{\times}$, we have

(1) $p^{n\left(\frac{k-2}{2}\right)} f_{\mathbf{v}_{m}}\left(x_{n}(a)\right)\left(\overline{a_{p}} / a_{p}\right)^{m} \in \frac{1}{(k-2) !} A$;

(2) the congruence relation

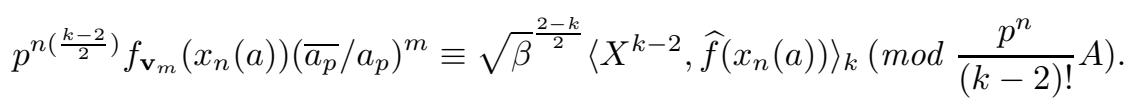

Proof. We write $a=\left(a^{(p)}, a_{p}\right) \in\left(\widehat{K}^{(p)}\right)^{\times} \times K_{p}^{\times}$. By definition, we have

$$
f_{\mathbf{v}_{m}}\left(x_{n}(a)\right)\left(\overline{a_{p}} / a_{p}\right)^{m}=\left\langle\rho_{k}\left(\gamma_{\mathfrak{p}}\right) \rho_{k, p}\left(\left(\varsigma_{p}^{(n)}\right)^{-1}\right) \mathbf{v}_{m}^{*}, \widehat{f}\left(x_{n}(a)\right)\right\rangle_{k} .
$$

Here we are making use of the fact that $\rho_{k, p}(t)$ acts on $\mathbf{v}_{m}$ by $(\bar{t} / t)^{m}$ for $t \in\left(K \otimes \mathbf{Q}_{p}\right)^{\times}$. A direct computation shows that $\rho_{k}\left(\gamma_{\mathfrak{p}}^{-1}\right)\left(\rho_{k, p}\left(\left(\varsigma_{p}^{(n)}\right)^{-1}\right)=\rho_{k}\left(Z_{p}\right)\right.$, where

$$
Z_{p}=\left(\begin{array}{cc}
1 & \sqrt{\beta} \\
0 & p^{n} \sqrt{\beta} \delta
\end{array}\right) \text { if } p \text { is split in } K
$$

and

$$
Z_{p}=\left(\begin{array}{cc}
1 & \sqrt{\beta} \\
-p^{n} \boldsymbol{\theta} & -p^{n} \sqrt{\beta} \boldsymbol{\theta}
\end{array}\right) \text { if } p \text { is non-split in } K
$$

Note that $\operatorname{det} Z_{p}=\sqrt{\beta} p^{n} \delta$,

$$
Z_{p} \in M_{2}\left(\mathcal{O}_{K_{p}}\right), \quad Z_{p} \equiv\left(\begin{array}{cc}
1 & \sqrt{\beta} \\
0 & 0
\end{array}\right)\left(\bmod p^{n} M_{2}\left(\mathcal{O}_{K_{p}}\right)\right) .
$$

For $P(X, Y) \in L_{k}(A)$, we find that

$$
\begin{aligned}
p^{\frac{n(k-2)}{2}} D_{K}^{\frac{k-2}{2}} \cdot \rho_{k}\left(\gamma_{\mathfrak{p}}^{-1}\right) \rho_{k, p}\left(\left(\varsigma_{p}^{(n)}\right)^{-1}\right) P(X, Y) & =p^{\frac{n(k-2)}{2}} D_{K}^{\frac{k-2}{2}} P\left((X, Y) Z_{p}\right)\left(\operatorname{det} Z_{p}\right)^{\frac{2-k}{2}} \\
& =\sqrt{\beta}^{\frac{2-k}{2}} P\left((X, Y) Z_{p}\right) \in L_{k}(A) .
\end{aligned}
$$

In particular,

$$
p^{\frac{n(k-2)}{2}} \rho_{k}\left(\gamma_{\mathfrak{p}}^{-1}\right) \rho_{k, p}\left(\left(\varsigma_{p}^{(n)}\right)^{-1}\right) \mathbf{v}_{m}^{*} \equiv \sqrt{\beta}^{\frac{2-k}{2}} X^{k-2}\left(\bmod p^{n} L_{k}(A)\right) .
$$

In view of (4.4), the assertions of the proposition follow immediately.

We make the following ordinary hypothesis:

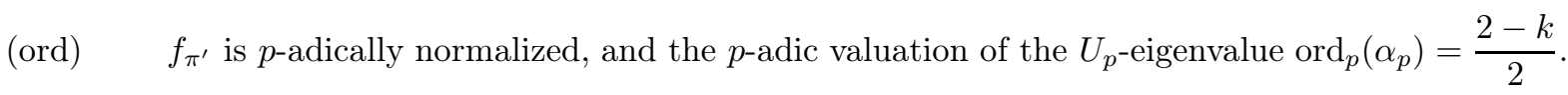

Corollary 4.5. Suppose (ord) holds. Then $\Theta_{n}^{[m]}\left(f_{\pi^{\prime}}^{\dagger}\right) \in[(k-2) !]^{-1} \mathcal{O}_{\pi, p}\left[\mathcal{G}_{n}\right]$. Moreover,

$$
\Theta_{n}^{[m]}\left(f_{\pi^{\prime}}^{\dagger}\right) \equiv \Theta_{n}^{[0]}\left(f_{\pi^{\prime}}^{\dagger}\right)\left(\bmod p^{n}[(k-2) !]^{-1} \mathcal{O}_{\pi, p}\left[\mathcal{G}_{n}\right]\right) .
$$


Proof. By definition, for $a \in \widehat{K}^{\times}$we have

$$
\alpha_{p}^{-n}\left(\widehat{\varphi}_{\pi^{\prime}}^{[m]}\right)^{\dagger}(a)=\alpha_{p}^{-n} \varphi_{\pi^{\prime}}^{[m]}\left(x_{n}(a)\right)\left(\overline{a_{p}} / a_{p}\right)^{m}-\alpha_{p}^{-2}\left(\alpha_{p}^{-(n-1)} \varphi_{\pi^{\prime}}^{[m]}\left(x_{n-1}(a)\right)\left(\overline{a_{p}} / a_{p}\right)^{m}\right) .
$$

Applying Lemma 4.4 to $f=f_{\pi^{\prime}}$, we find that

- $\alpha_{p}^{-n}\left(\widehat{\varphi}_{\pi^{\prime}}^{[m]}\right)^{\dagger}(a) \in \mathcal{O}_{\pi, p}$,

- $\alpha_{p}^{-n}\left(\widehat{\varphi}_{\pi^{\prime}}^{[m]}\right)^{\dagger}(a)\left(\bmod p^{n}[(k-2) !]^{-1}\right)$ is independent of $m$.

The corollary follows immediately.

Let $\mathcal{G}_{\infty}:=\lim _{n} \mathcal{G}_{n}$. Let $\Gamma^{-} \simeq \mathbf{Z}_{p}$ be the maximal $\mathbf{Z}_{p}$-free quotient group of $\mathcal{G}_{\infty}$ and let $\Delta$ be the torsion subgroup of $\mathcal{G}_{\infty}$. We have an exact sequence

$$
0 \longrightarrow \Delta \longrightarrow \mathcal{G}_{\infty} \longrightarrow \Gamma^{-} \longrightarrow 0 .
$$

Fix a non-canonical isomorphism $\mathcal{G}_{\infty} \simeq \Delta \times \Gamma^{-}$once and for all. If $n \geq 1$, the map $\Delta \rightarrow \mathcal{G}_{\infty} \rightarrow \mathcal{G}_{n}$ is injective, and hence

$$
\mathcal{G}_{n} \simeq \Delta \times \Gamma_{n}^{-}, \Gamma^{-} \rightarrow \Gamma_{n}^{-}:=\mathcal{G}_{n} / \Delta
$$

Let $\chi_{t}$ be a branch character, i.e. a character $\chi_{t}: \Delta \rightarrow \overline{\mathbf{Q}}^{\times}$and let $\mathcal{O}=\mathcal{O}_{\pi, p}\left[\chi_{t}\right]$. Define the $\chi_{t}$-branch of $\Theta_{n}^{[0]}\left(f_{\pi^{\prime}}^{\dagger}\right)$ by

We define

$$
\Theta_{n}\left(f_{\pi^{\prime}}^{\dagger}, \chi_{t}\right):=\chi_{t}\left(\Theta_{n}^{[0]}\left(f_{\pi^{\prime}}^{\dagger}\right)\right) \in[(k-2) !]^{-1} \mathcal{O}\left[\Gamma_{n}^{-}\right]
$$

$\Theta_{\infty}(\pi):=\left\{\Theta_{n}^{[0]}\left(f_{\pi^{\prime}}^{\dagger}\right)\right\}_{n} \in[(k-2) !]^{-1} \mathcal{O} \llbracket \mathcal{G}_{\infty} \rrbracket ; \quad \Theta_{\infty}\left(\pi, \chi_{t}\right):=\left\{\Theta_{n}\left(f_{\pi^{\prime}}^{\dagger}, \chi_{t}\right)\right\}_{n}=\chi_{t}\left(\Theta_{\infty}(\pi)\right) \in[(k-2) !]^{-1} \mathcal{O} \llbracket \Gamma^{-} \rrbracket$.

Let $\mathfrak{X}_{p}^{\text {crit }}$ be the set of critical specializations defined in the introduction. Note that $\mathfrak{X}_{p}^{\text {crit }}$ consists of the $p$-adic avatars of Hecke characters $\chi$ of $p$-power conductor satisfying (crit) and trivial on $\Delta$.

Theorem 4.6. Let $\widehat{\nu} \in \mathfrak{X}_{p}^{\text {crit }}$ be a p-adic character of weight $(m,-m)$ and conductor $p^{s}$. We have the interpolation formula

$$
\begin{aligned}
\widehat{\nu}\left(\Theta_{\infty}\left(\pi, \chi_{t}\right)^{2}\right)= & \Gamma\left(\frac{k}{2}+m\right) \Gamma\left(\frac{k}{2}-m\right) \cdot \frac{L\left(f_{\pi} / K, \chi_{t} \nu, \frac{k}{2}\right)}{\Omega_{\pi, N^{-}}} \cdot e_{p}\left(\pi, \chi_{t} \nu\right)^{2-\operatorname{ord}_{p}(N)} \cdot p^{s} A_{p}^{-2 s}\left(p^{s} D_{K}\right)^{k-2} \\
& \times u_{K}^{2} \sqrt{D_{K}} \cdot \epsilon\left(\pi_{p}\right)(-1)^{m} \prod_{q \mid\left(D_{K}, N^{-}\right), q=\mathfrak{q}^{2}}\left(1-\epsilon\left(\pi_{q}\right) \chi_{t}(\mathfrak{q})\right) \cdot \chi_{t} \nu\left(\mathfrak{N}^{+}\right) .
\end{aligned}
$$

Proof. Let $n_{0}=\max \{s, 1\}$. For each integer $r>n_{0}$, we choose $n>r$ such that $\widehat{\nu}\left(\bmod p^{r}\right)$ is trivial on $\widehat{\mathcal{O}}_{n}^{\times}$. Let $\chi=\chi_{t} \nu$. By Corollary 4.5, we have

$$
\widehat{\nu}\left(\Theta_{\infty}\left(\pi, \chi_{t}\right)\right) \equiv \widehat{\chi}\left(\Theta_{n}^{[0]}\left(f_{\pi^{\prime}}^{\dagger}\right)\right) \equiv \widehat{\chi}\left(\Theta_{n}^{[m]}\left(f_{\pi^{\prime}}^{\dagger}\right)\right)=\widehat{\chi}\left(\Theta_{n_{0}}^{[m]}\left(f_{\pi^{\prime}}^{\dagger}\right)\right)\left(\bmod p^{r}[(k-2) !]^{-1}\right) .
$$

This congruence relation holds for all $r>n_{0}$. Therefore, $\widehat{\nu}\left(\Theta_{\infty}\left(\pi, \chi_{t}\right)\right)=\widehat{\chi}\left(\Theta_{n_{0}}^{[m]}\left(f_{\pi^{\prime}}^{\dagger}\right)\right)$, and the theorem follows from Proposition 4.3

Remark 4.7. The theta element $\Theta_{\infty}\left(\pi, \chi_{t}\right)$ is the square root of the anticyclotomic $p$-adic $L$-function associated to $\left(\pi, \chi_{t}\right)$. In view of the evaluation formula, we assume the following local root number condition in the remainder of this article:

$$
\epsilon\left(\pi_{q}\right) \chi_{t}(\mathfrak{q})=-1 \text { for every } q \mid\left(D_{K}, N^{-}\right) \text {with } q=\mathfrak{q}^{2} .
$$

Note that $(\mathrm{ST})$ is always satisfied if $\left(D_{K}, N^{-}\right)=1$.

Let $*: \mathcal{O} \llbracket \mathcal{G}_{\infty} \rrbracket \rightarrow \mathcal{O} \llbracket \mathcal{G}_{\infty} \rrbracket$ be the involution defined by $\sigma \mapsto \sigma^{-1}$. We show that $\Theta_{\infty}(\pi)$ satisfies the functional equation in the following sense.

Theorem 4.8. Let $r_{0}$ be the number of prime divisors of $\left(D_{K}, N^{-}\right)$. Let

$$
\epsilon^{\prime}:=(-1)^{r_{0}+\frac{k}{2}} \prod_{q \nmid p D_{K}} \epsilon\left(\pi_{q}\right) \in\{ \pm 1\}
$$

and let $\sigma_{\mathfrak{N}^{+}}=\left\{\left[\mathfrak{N}^{+}\right]_{n}\right\}$ be the image of $\mathfrak{N}^{+}$in $\mathcal{G}_{\infty}$. We have the functional equation:

$$
\Theta_{\infty}(\pi)^{*}=\epsilon^{\prime} \cdot \Theta_{\infty}(\pi) \cdot \sigma_{\mathfrak{N}^{+}}^{-1} .
$$


ProOF. Let $\varphi_{\pi^{\prime}}=\varphi_{\pi^{\prime}}^{[0]}$. Using the automorphy of $\varphi_{\pi^{\prime}}$, we have

$$
\left.\varphi_{\pi^{\prime}}\left(x_{n}\left(a^{-1}\right)\right)=\varphi_{\pi^{\prime}}^{\dagger}\left(x_{n}(\bar{a})\right)=\varphi_{\pi^{\prime}}\left(x_{n}(a) J_{\varsigma, n}\right) \quad\left(J_{\varsigma}^{(n)}=\left(\varsigma^{(n)}\right)^{-1} J_{\varsigma}^{(n)}\right)\right) .
$$

By the choice of $J$ and Lemma 3.7 it is straightforward to show that

$$
\pi\left(J_{\varsigma, n}\right) \varphi_{\pi^{\prime}}(Q)=\epsilon^{\prime} \cdot \varphi_{\pi^{\prime}}\left(\sigma_{\mathfrak{N}^{+}}(Q)\right) \text { for } Q \in \mathcal{G}_{n} .
$$

Therefore,

$$
\Theta_{n}(\pi)^{*}=\sum_{\sigma \in \mathcal{G}_{n}} \varphi_{\pi^{\prime}}\left(\sigma^{-1}\left(P_{n}^{\dagger}\right)\right) \sigma=\epsilon^{\prime} \cdot \Theta_{n}(\pi) \cdot\left[\mathfrak{N}^{+}\right]_{n}^{-1} .
$$

This proves the theorem.

Remark. Note that $\epsilon\left(\pi_{\infty}\right)=(-1)^{\frac{k}{2}}$. If $\chi_{t}=\mathbf{1}$ is the trivial character, then $\epsilon\left(\pi_{q}\right)=-1$ for all $q \mid N^{-}$by (ST). It follows that

$$
\epsilon^{\prime}=(-1)^{\frac{k}{2}} \prod_{q \neq p} \epsilon\left(\pi_{q}\right)=\epsilon(\pi) \epsilon\left(\pi_{p}\right) .
$$

\section{THE NON-VANiSHING OF THETA ELEMENTS MODULO $\ell$}

5.1. We retain the notation in the previous section. Throughout, we suppose that $f_{\pi^{\prime}}$ is $\lambda$-adically normalized. The purpose of this section is to study the non-vanishing properties of theta elements $\left\{\Theta_{n}\left(f_{\pi^{\prime}}^{\dagger}, \chi_{t}\right)\right\}_{n}$ modulo $\lambda$. Let $\Delta^{\text {alg }}$ be the subgroup of $\mathcal{G}_{\infty}$ generated by the image of $K_{\text {ram }}^{\times}:=\prod_{q \mid D_{K}} K_{q}^{\times}$. It is clear that $\Delta^{\text {alg }}$ is a $(2, \cdots, 2)$-subgroup of $\Delta$. Let $\mathcal{D}_{0}$ be a set of representatives of $\Delta^{\text {alg }}$ in $K_{\text {ram }}^{\times}$. Choose an arbitrary set $\mathcal{D}_{1}$ of representatives of $\Delta / \Delta^{\text {alg }}$ in $\widehat{K}^{\times}$. Then $\mathcal{D}:=\mathcal{D}_{1} \mathcal{D}_{0}$ be a set of representatives of $\Delta$ in $\widehat{K}^{\times}$. By definition, we can write

$$
\Theta_{n}\left(f_{\pi^{\prime}}^{\dagger}, \chi_{t}\right)=\sum_{[u]_{n} \in \Gamma_{n}^{-}} \sum_{\tau \in \mathcal{D}_{1}} \chi_{t}(\tau)\left(\alpha_{p}^{-n} \sum_{d \in \mathcal{D}_{0}} \varphi_{\pi^{\prime}}^{\dagger}\left(x_{n}(\tau u) d\right) \chi_{t}(d)\right) \cdot[u]_{n} \quad\left(\varphi_{\pi^{\prime}}^{\dagger}=\Psi\left(\mathbf{v}_{0}^{*} \otimes f_{\pi^{\prime}}^{\dagger}\right)\right) .
$$

5.2. Uniform distribution of CM points. We recall a crucial result in [CV05]. Let $\bar{K}^{\times}$the closure of $K^{\times}$ in $\widehat{K}^{\times}$and let $\bar{B}^{\times}$be the closure of $B^{\times}$in $\widehat{B}^{\times}$. Let $\mathrm{CM}:=\bar{K}^{\times} \backslash \widehat{B}^{\times}, \mathcal{X}:=\bar{B}^{\times} \backslash \widehat{B}^{\times}$and $\mathcal{Z}:=\overline{\mathbf{Q}}_{+} \backslash \widehat{\mathbf{Q}}^{\times}$. The group $\widehat{B}^{\times}$acts on these spaces by the right translation and $\widehat{K}^{\times}$acts on CM and $\mathcal{Z}$ by the left multiplication.

Let Red : $\mathrm{CM} \rightarrow \mathcal{X}$ be the natural quotient map and let $c: \mathcal{X} \rightarrow \mathcal{Z}$ be the map induced by the reduced norm $\mathrm{N}: B^{\times} \rightarrow \mathbf{Q}^{\times}$. For $g \in \widehat{B}^{\times}$, let $[g]$ denote the image of $g$ in $\mathrm{CM}$. Let $\mathcal{U}$ be an open compact subgroup of $\widehat{B}^{\times}$. Put

$$
\mathcal{X}\left(\mathcal{D}_{1}, \mathcal{U}\right)=\prod_{\tau \in \mathcal{D}_{1}} \mathcal{X} / \mathcal{U} \text { and } \mathcal{Z}\left(\mathcal{D}_{1}, \mathcal{U}\right)=\prod_{\tau \in \mathcal{D}_{1}} \mathcal{Z} / \mathrm{N}(\mathcal{U}) .
$$

Define

$$
\operatorname{Red}_{\mathcal{D}_{1}}: \mathrm{CM} \longrightarrow \mathcal{X}\left(\mathcal{D}_{1}, \mathcal{U}\right), \quad x \mapsto(\operatorname{Red}(\tau \cdot x) \mathcal{U})_{\tau \in \mathcal{D}_{1}}
$$

and

$$
c_{\mathcal{D}_{1}}: \mathcal{X}\left(\mathcal{D}_{1}, \mathcal{U}\right) \longrightarrow \mathcal{Z}\left(\mathcal{D}_{1}, \mathcal{U}\right), \quad\left(x_{\tau}\right)_{\tau \in \mathcal{D}_{1}} \mapsto\left(\mathrm{N}\left(x_{\tau}\right)\right)_{\tau \in \mathcal{D}_{1}} .
$$

The following proposition is a special case of [CV05, Corollary 2.10].

Proposition 5.1. Let $\mathcal{H}$ be a $B_{p}^{\times}$-orbit in $\mathrm{CM}$ and let $\overline{\mathcal{H}}$ be the image of $\mathcal{H}$ in $\mathrm{CM} / \mathcal{U}$. Then for all but finitely many $x \in \overline{\mathcal{H}}$, we have

where $\bar{x}=c_{\mathcal{D}_{1}} \circ \operatorname{Red}_{\mathcal{D}_{1}}(x)$.

$$
\operatorname{Red}_{\mathcal{D}_{1}}\left(\widehat{\mathcal{O}}_{K}^{\times} \cdot x\right)=c_{\mathcal{D}_{1}}^{-1}\left(\widehat{\mathcal{O}}_{K}^{\times} \cdot \bar{x}\right),
$$

Proof. This is [CV05, Corollary 2.10] with $\mathfrak{S}=\{\emptyset\}$ and $\mathfrak{R}=\mathcal{D}_{1}$.

The following corollary is a immediate consequence of the above proposition.

Corollary 5.2. Let $\left\{\beta_{\tau}\right\}_{\tau \in \mathcal{D}_{1}}$ be a sequence in $A$ such that $\beta_{\tau_{1}} \in A^{\times}$for some $\tau_{1}$. Let $f \in \mathcal{M}_{2}(\mathcal{U}, A)$. Suppose that

(1) $f$ is not Eisenstein; 
(2) $\mathrm{N}(\mathcal{U}) \supset \prod_{q \mid D_{K}} \mathbf{Z}_{q}^{\times}$.

Then there exists an integer $n_{0}$ such that for every $n>n_{0}$, we have

$$
\sum_{\tau \in \mathcal{D}_{1}} \beta_{\tau} \cdot f\left(x_{n}(a \tau)\right) \neq 0 \text { for some } a \in \widehat{K}^{\times} .
$$

Proof. Let $P_{0}:=\left[\varsigma^{(0)}\right] \in$ CM. Let $\mathcal{H}=P_{0} \cdot B_{p}^{\times}$be the $B_{p}^{\times}$-orbit of $P_{0}$. Then $P_{n}:=P_{0} \cdot\left(\begin{array}{cc}p^{n} & 0 \\ 0 & 1\end{array}\right) \in \mathcal{H}$, and from (2.9) we find that the image of $\left\{P_{n}\right\}_{n=1,2, \ldots}$ are distinct in $\widehat{K}^{\times} \backslash \widehat{B}^{\times} / \widehat{R}^{\times}$. By Proposition [5.1, there exists $n_{0}$ such that

$$
\operatorname{Red}_{\mathcal{D}_{1}}\left(\widehat{\mathcal{O}}_{K}^{\times} P_{n}\right)=c_{\mathcal{D}_{1}}^{-1}\left(\widehat{\mathcal{O}}_{K}^{\times} \overline{P_{n}}\right) \text { for every } n>n_{0} .
$$

Fix $n>n_{0}$. Since $f$ is not Eisenstein, $f(y) \neq f(z)$ for some $y, z \in \mathcal{X}$ with $c(y)=c(z)$. The assumption (2) implies that the norm map $\mathrm{N}: \widehat{K}^{\times} \rightarrow \mathcal{Z} / \mathrm{N}(\mathcal{U})$ is surjective as the class number of $\mathbf{Q}$ is one. Hence, replacing $\mathcal{D}_{1}$ by $a^{\prime} \mathcal{D}_{1}$ for some $a^{\prime} \in \widehat{K}^{\times}$if necessary, we may assume

$$
c(y)=c(z)=c\left(\operatorname{Red}\left(P_{n}\right)\right)(\bmod \mathrm{N}(\mathcal{U})) .
$$

Take $\left(w_{\tau}\right)_{\tau \in \mathcal{D}_{1}} \in c_{\mathcal{D}_{1}}^{-1}\left(\overline{P_{n}}\right)$. By (5.2), there exist $a_{1}, a_{2} \in \widehat{\mathcal{O}}_{K}^{\times}$such that

$$
\operatorname{Red}_{\mathcal{D}_{1}}\left(a_{1} P_{n}\right)=\left(y, w_{\tau_{2}}, \cdots\right) ; \operatorname{Red}_{\mathcal{D}_{1}}\left(a_{2} P_{n}\right)=\left(z, w_{\tau_{2}}, \cdots\right) .
$$

It follows that

$$
\sum_{\tau \in \mathcal{D}_{1}} \beta_{\tau} \cdot f\left(x_{n}\left(a_{1} \tau\right)\right)-\sum_{\tau \in \mathcal{D}_{1}} \beta_{\tau} \cdot f\left(x_{n}\left(a_{2} \tau\right)\right)=\beta_{\tau_{1}}(f(y)-f(z)) \neq 0 .
$$

It is clear that either $a_{1}$ or $a_{2}$ does the job.

5.3. Eisenstein functions. Let $A$ be a $\mathbf{Z}$-algebra. Let $U$ be an open-compact subgroup of $\widehat{B}^{\times}$. Denote by $\mathcal{M}_{2}(U, A)$ the set of functions $h: B^{\times} \backslash \widehat{B}^{\times} \rightarrow A$ such that $h$ is right invariant by $U$. Let $\mathcal{M}_{2}(A):=$ $\lim _{U \subset \widehat{B}^{\times}} \mathcal{M}_{2}(U, A)$ be the space of smooth $A$-valued functions on $B^{\times} \backslash \widehat{B}^{\times}$. We write $\varrho: \widehat{B}^{\times} \rightarrow$ Aut $\mathcal{M}_{2}(A)$ for the right translation of $\widehat{B}^{\times}$.

Definition 5.3. Let $B^{1}=\left\{g \in B^{\times} \mid \mathrm{N}(g)=1\right\}$ be an algebraic group over Q. Put

$$
\mathcal{M}_{2}(A)_{\text {Eis }}:=\left\{h \in \mathcal{M}_{2}(A) \mid \varrho\left(g_{1}\right) h=h \text { for all } g_{1} \in B^{1}\left(\mathbf{A}_{f}\right)\right\} .
$$

It is clear that $\mathcal{M}_{2}(A)_{\text {Eis }}$ is a $\widehat{B}^{\times}$-invariant subspace of $\mathcal{M}_{2}(A)$. Let

$$
\mathcal{S}_{2}(A):=\mathcal{M}_{2}(A) / \mathcal{M}_{2}(A)_{\text {Eis }} .
$$

Let $\mathcal{S}_{2}(U, A)$ denote the image of $\mathcal{M}_{2}(U, A)$ in $\mathcal{S}_{2}(A)$.

A function $h \in \mathcal{M}_{2}(A)$ is called Eisenstein if $h \in \mathcal{M}_{2}(A)_{\text {Eis. }}$. Equivalently, $h$ is Eisenstein if and only $h(g)=h_{1}(\mathrm{~N}(g))$ for some smooth function $h_{1}: \mathbf{Q}_{+}^{\times} \backslash \widehat{\mathbf{Q}}^{\times} \rightarrow A$.

We make the following observations in the flavor of Ihara's lemma. The first one is taken from Vat03, Proposition 5.3].

Lemma 5.4. Let $q \nmid N^{-}$be a finite place. Let $t_{q} \in B_{q}^{\times}$such that $\mathrm{N}\left(t_{q}\right)=q$. Let $\mathcal{R}^{\prime} \in$ End $\mathcal{M}_{2}(A)$ be the endomorphism defined by

$$
\mathcal{R}^{\prime}=1+\beta \cdot \varrho\left(t_{q}\right) \quad(\beta \in A) .
$$

Suppose that $U \supset R_{q}^{\times}:=\left(R \otimes \mathbf{z} \mathbf{Z}_{q}\right)^{\times}=\mathrm{GL}_{2}\left(\mathbf{Z}_{q}\right)$. Then $\mathcal{R}^{\prime}: \mathcal{S}_{2}(U, A) \rightarrow \mathcal{S}_{2}(A)$ is injective.

Proof. Let $h \in \mathcal{M}_{2}(U, A)$. If $\mathcal{R}(h) \in \mathcal{M}_{2}(A)_{\text {Eis }}$, then it is easy to see that $h$ is right invariant by $\operatorname{SL}_{2}\left(\mathbf{Z}_{q}\right)$ and $t_{q} \mathrm{SL}_{2}\left(\mathbf{Z}_{q}\right) t_{q}^{-1}$. By a theorem of Ihara, $h$ is right invariant by $\mathrm{SL}_{2}\left(\mathbf{Q}_{q}\right)$ and hence by $B_{1}\left(\mathbf{A}_{f}\right)$ in virtue of the strong approximation theorem for $B_{1}$.

Lemma 5.5. Let $q \nmid N^{-}$be a finite place. Let $\beta_{1}, \cdots, \beta_{s} \in A$ and let $\mathcal{R} \in \operatorname{End}\left(\mathcal{M}_{2}(A)\right)$ be the endomorphism defined by

$$
\mathcal{R}=1+\sum_{i=1}^{s} \beta_{i} \cdot \varrho\left(\left(\begin{array}{cc}
q^{-i} & 0 \\
0 & 1
\end{array}\right)\right) .
$$

Then $\mathcal{R}: \mathcal{S}_{2}(U, A) \rightarrow \mathcal{S}_{2}(A)$ is injective. 
Proof. Let $h \in \mathcal{M}_{2}(U, A)$. We need to show that if

$$
\mathcal{R}(h)=h+\sum_{i=1}^{s} \beta_{i} \cdot \varrho\left(\left(\begin{array}{cc}
q^{-i} & 0 \\
0 & 1
\end{array}\right)\right) h \in \mathcal{M}_{2}(A)_{\text {Eis }},
$$

then $h \in \mathcal{M}_{2}(A)_{\text {Eis }}$. Let $S_{h} \supset U$ be the stabilizer of $h$ in $\widehat{B}^{\times}$. Namely,

$$
S_{h}:=\left\{g \in \widehat{B}^{\times} \mid \varrho(g) h=h\right\} .
$$

Let $N\left(\mathbf{Q}_{q}\right)$ be the unipotent radical of the upper triangular subgroup in $\mathrm{GL}_{2}\left(\mathbf{Q}_{q}\right)$ and let

$$
N^{\prime}:=N\left(\mathbf{Q}_{q}\right) \cap U \subset S_{h} .
$$

Write $u:=\left(\begin{array}{cc}q^{-1} & 0 \\ 0 & 1\end{array}\right)$ and

$$
P(u)=-\sum_{i=1}^{s} \beta_{i} \varrho(u)^{i-1} \in \operatorname{End} \mathcal{M}_{2}(A)^{N^{\prime}} .
$$

By the assumption that $h-\varrho(u) P(u) h \in \mathcal{M}_{2}(A)_{\text {Eis }}$, we find that for every positive integer $n$,

$$
h-\varrho\left(u^{n}\right) P(u)^{n} \cdot h \in \mathcal{M}_{2}(A)_{\text {Eis }} .
$$

Since $h, P(u)^{n} h \in \mathcal{M}_{2}(A)^{N^{\prime}}$ and $N^{\prime}$ is a proper subgroup, using the identity $u^{-n}\left(\begin{array}{ll}1 & x \\ 0 & 1\end{array}\right) u^{n}=\left(\begin{array}{cc}1 & q^{n} x \\ 0 & 1\end{array}\right)$, we deduce from (5.3) that

$$
\left(\begin{array}{ll}
1 & x \\
0 & 1
\end{array}\right) \in S_{h} \text { for all } x \in \mathbf{Q}_{q}
$$

On the other hand, $\left(\begin{array}{ll}1 & 0 \\ y & 1\end{array}\right) \in S_{h}$ for some $y \in \mathbf{Q}_{q}^{\times}$. By the relation

$$
\left(\begin{array}{ll}
1 & 0 \\
y & 1
\end{array}\right)=\left(\begin{array}{cc}
1 & y^{-1} \\
0 & 1
\end{array}\right)\left(\begin{array}{cc}
0 & y^{-1} \\
-y & 0
\end{array}\right)\left(\begin{array}{cc}
1 & y^{-1} \\
0 & 1
\end{array}\right)
$$

we find $w_{0}=\left(\begin{array}{cc}0 & y^{-1} \\ -y & 0\end{array}\right) \in S_{h}$, and hence

$$
\left(\begin{array}{ll}
1 & 0 \\
x & 1
\end{array}\right)=w_{0}^{-1}\left(\begin{array}{cc}
1 & -y^{2} x \\
0 & 1
\end{array}\right) w_{0} \in S_{h}
$$

It follows that $S_{h}$ contains $\mathrm{SL}_{2}\left(\mathbf{Q}_{q}\right) \cdot U$. By the strong approximation for $B^{1}$, we find that $B^{1}\left(\mathbf{A}_{f}\right) \subset S_{h}$.

Let $\rho_{\pi, \lambda}: \operatorname{Gal}(\overline{\mathbf{Q}} / \mathbf{Q}) \rightarrow \mathrm{GL}_{2}\left(\mathcal{O}_{\pi, \ell}\right)$ be the $\ell$-adic Galois representation attached to $f_{\pi}$.

Lemma 5.6. Suppose that the residual Galois representation $\bar{\rho}_{\pi, \lambda}$ is irreducible and $\ell>k-2$. Let $\mathbf{v} \in$ $L_{k}\left(\mathcal{O}_{\pi, \ell}\right)$. If $\mathbf{v} \not \equiv 0(\bmod \lambda)$, the function $f_{\mathbf{v}}(g):=\left\langle\mathbf{v}, \widehat{f}_{\pi^{\prime}}(g)\right\rangle_{k}(\bmod \lambda) \in \mathcal{M}_{2}\left(\mathcal{O}_{\pi, \ell} / \lambda\right)$ is not Eisenstein.

Proof. We note that $f_{\mathbf{v}}$ is not a zero function by the irreducibility of $L_{k}\left(\mathcal{O}_{\pi, \ell} / \lambda\right)$ as $\mathrm{GL}_{2}\left(\mathcal{O}_{\pi, \ell} / \lambda\right)$-module when $\ell>k-2$. Therefore, if $f_{\mathbf{v}}$ is Eisenstein, then $\bar{\rho}_{\pi, \lambda}$ is reducible.

5.4. The vanishing of $\mu$-invariants. For each positive integer $s$, define the open compact subgroup $\mathcal{I}_{1}\left(p^{s}\right)$ of $\widehat{R}^{\times}$by

$$
\mathcal{I}_{1}\left(p^{s}\right):=\left\{g \in \widehat{R}^{\times} \mid g_{p} \equiv\left(\begin{array}{ll}
1 & * \\
0 & 1
\end{array}\right)\left(\bmod p^{s}\right)\right\} .
$$

Let $\varpi$ be a generator of the maximal ideal of $\mathcal{O}:=\mathcal{O}_{\pi, \ell}\left[\chi_{t}\right]$. Suppose that $\ell=p$. We follow the approach of Vatsal to study the $\mu$-invariant of $\Theta_{\infty}\left(\pi, \chi_{t}\right) \in \mathcal{O} \llbracket \Gamma^{-} \rrbracket$.

Theorem 5.7. Let $r_{0}$ be as in Theorem 4.8, In addition to (ST) and (ord), we assume that

(1) $p>k-2$ and $p \nmid 2^{r_{0}}$,

(2) $\bar{\rho}_{\pi, \lambda}$ is absolutely irreducible.

Then Iwasawa $\mu$-invariant of $\Theta_{\infty}\left(\pi, \chi_{t}\right)$ vanishes. 
Proof. We define a function $\mathbf{f}_{p}: B^{\times} \backslash \widehat{B}^{\times} / \widehat{\mathbf{Q}}^{\times} \rightarrow \mathcal{O} / \varpi \mathcal{O}$ by

$$
\mathbf{f}_{p}(g)=\sqrt{\beta}^{\frac{2-k}{2}} \cdot\left\langle X^{k-2}, \widehat{f}_{\pi^{\prime}}(g)\right\rangle_{k}(\bmod \varpi)
$$

A direct computation shows that $\mathbf{f}_{p} \in \mathcal{M}_{2}\left(\mathcal{I}_{1}(p), \mathcal{O} / \varpi \mathcal{O}\right)$. Let $\mathcal{U}_{\mathcal{D}_{0}}$ be the open-compact subgroup given by

$$
\mathcal{U}_{\mathcal{D}_{0}}=\left\{g \in \widehat{R}^{\times} \mid g_{q} \in R_{q}^{\times} \cap \varpi_{K_{q}} R_{q}^{\times} \varpi_{K_{q}}^{-1} \text { for all } q \mid D_{K}\right\},
$$

where $\varpi_{K_{q}}$ is a uniformizer of $K_{q}$. It is easy to see that

$$
\mathrm{N}\left(\mathcal{U}_{\mathcal{D}_{0}}\right) \supset \prod_{q \mid D_{K}} \mathbf{Z}_{q}^{\times}
$$

Let $\mathbf{f}_{\mathcal{D}_{0}} \in \mathcal{M}_{2}\left(\mathcal{U}_{\mathcal{D}_{0}} \cap \mathcal{I}_{1}(p), \mathcal{O} / \varpi \mathcal{O}\right)$ be the function defined by

$$
\mathbf{f}_{\mathcal{D}_{0}}(g)=\sum_{d \in \mathcal{D}_{0}} \mathbf{f}_{p}(g d) \chi_{t}(d)(\bmod \varpi) .
$$

From Lemma 4.4, we can deduce that

$$
\Theta_{n}\left(f_{\pi^{\prime}}^{\dagger}, \chi_{t}\right)(\bmod \varpi)=A_{p}^{-n} \sum_{[u]_{n} \in \Gamma_{n}^{-}}\left(\sum_{\tau \in \mathcal{D}_{1}} \mathbf{f}_{\mathcal{D}_{0}}^{\dagger}\left(x_{n}(u \tau)\right) \chi_{t}(\tau)\right) \cdot[u]_{n},
$$

where $\mathbf{f}_{\mathcal{D}_{0}}^{\dagger} \in \mathcal{M}_{2}(\mathcal{U}, \mathcal{O} / \varpi \mathcal{O})$ is given by

$$
\mathbf{f}_{\mathcal{D}_{0}}^{\dagger}:=\mathbf{f}_{\mathcal{D}_{0}}-p^{\frac{k-2}{2}} A_{p}^{-1} \cdot \varrho\left(\left(\begin{array}{cc}
p^{-1} & 0 \\
0 & 1
\end{array}\right)\right) \mathbf{f}_{\mathcal{D}_{0}}, \mathcal{U}:=\mathcal{U}_{\mathcal{D}_{0}} \cap \mathcal{I}_{1}\left(p^{2}\right) .
$$

To prove the vanishing of the $\mu$-invariant, we need to show that for $n \gg 0$, there exists $a \in \widehat{K}^{\times}$such that

$$
\sum_{\tau \in \mathcal{D}_{1}} \mathbf{f}_{\mathcal{D}_{0}}^{\dagger}\left(x_{n}(a \tau)\right) \chi_{t}(\tau) \not \equiv 0(\bmod \varpi)
$$

and in turn, it suffices to verify the assumptions for $\mathbf{f}_{\mathcal{D}_{0}}^{\dagger}$ in Corollary [5.2. By (5.5), $\mathrm{N}(\mathcal{U}) \supset \prod_{q \mid D_{K}} \mathbf{Z}_{q}^{\times}$. By Lemma 5.6] $\mathbf{f}_{p}$ is not Eisenstein, which implies that $\mathbf{f}_{\mathcal{D}_{0}}^{\dagger} \in \mathcal{M}_{2}(\mathcal{U}, \mathcal{O} / \varpi \mathcal{O})$ is not Eisenstein by the following Lemma 5.8 ,

Lemma 5.8. Suppose that $\mathbf{f}_{p}$ is not Eisenstein. Then $\mathbf{f}_{\mathcal{D}_{0}}^{\dagger}$ is not Eisenstein.

Proof. $\quad$ Let $q \mid D_{K}$ be a ramified place and $\varpi_{K_{q}}$ be a uniformizer of $K_{q}^{\times}$. Put

$$
\mathcal{R}_{q}^{\prime}:=1+\chi_{t}\left(\varpi_{K_{q}}\right) \varrho\left(\varpi_{K_{q}}\right) \in \operatorname{End}\left(\mathcal{M}_{2}(\mathcal{O} / \varpi \mathcal{O})\right) .
$$

Let $\left\{q_{i}\right\}_{i=1, \cdots s}$ are the set of prime divisors $q$ of $D_{K}$ with $q \nmid N^{-}$. By the assumption (ST), we have

$$
\mathbf{f}_{\mathcal{D}_{0}}=2^{r_{0}} \cdot \mathcal{R}_{q_{1}}^{\prime} \circ \mathcal{R}_{q_{2}}^{\prime} \cdots \circ \mathcal{R}_{q_{s}}^{\prime}\left(\mathbf{f}_{p}\right) \text {. }
$$

Applying Lemma 5.4 and Lemma 5.5 we conclude that $\mathbf{f}_{\mathcal{D}_{0}}^{\dagger}=\mathcal{R}_{p}\left(\mathbf{f}_{\mathcal{D}_{0}}\right)$ with $\mathcal{R}_{p}:=1-\alpha_{p}^{2} A_{p} \cdot \varrho\left(\left(\begin{array}{cc}p^{-1} & 0 \\ 0 & 1\end{array}\right)\right)$ is not Eisenstein if $\mathbf{f}_{p} \notin \mathcal{M}_{2}(\mathcal{O} / \varpi \mathcal{O})_{\text {Eis }}$.

5.5. The non-vanishing modulo $\ell$ with anticyclotomic twists. Suppose that $\ell \neq p$. We prove the nonvanishing of central $L$-values modulo $\ell$ with anticyclotomic twists, using a Galois average trick of Sinnot in [Sin87].

Theorem 5.9. Let $\chi$ be an anticyclotomic Hecke character of conductor $p^{s_{0}}$ and weight $(m,-m)$ with $-k / 2<$ $m<k / 2$. Suppose that

(1) $(\pi, \chi)$ satisfies (ST),

(2) $\ell \nmid 2^{r_{0}} p N D_{K}$ and $\ell>k-2$,

(3) $\bar{\rho}_{\pi, \lambda}$ is absolutely irreducible.

Then for all but finitely many $\nu: \Gamma^{-} \rightarrow \boldsymbol{\mu}_{p^{\infty}}$, we have

$$
\frac{L\left(f_{\pi} / K, \chi \nu, \frac{k}{2}\right)}{\Omega_{\pi, N^{-}}} \not \equiv 0(\bmod \lambda) .
$$


Proof. Choose a finite extension $\mathcal{O}$ of $\mathbf{Z}_{\ell}$ in $\mathbf{C}_{\ell}$ so that $\mathcal{O}$ contains $\mathcal{O}_{\pi, \ell}$ and the values of $\chi$ on $\mathbf{A}_{K, f}^{\times}$ and let $\varpi$ be a uniformizer of $\mathcal{O}$. Let $\widehat{f}_{\pi^{\prime}}^{\dagger}$ be the $\ell$-adic avatar of the $p$-stabilization $f_{\pi^{\prime}}^{\dagger}$. Define a function $F_{\ell}: B^{\times} \backslash \widehat{B}^{\times} \rightarrow \mathcal{O}$ by

$$
F_{\ell}(g)=\left\langle\rho_{k}\left(\gamma_{\mathfrak{l}}^{-1}\right) \mathbf{v}_{m}^{*}, \hat{f}_{\pi^{\prime}}^{\dagger}(g)\right\rangle_{k} \quad\left(\mathbf{v}_{m}^{*}=D_{K}^{\frac{2-k}{2}} \cdot X^{\frac{k-2}{2}-m} Y^{\frac{k-2}{2}+m}\right) .
$$

Note that $\gamma_{\mathfrak{l}} \in \mathrm{GL}_{2}\left(\mathcal{O}_{K_{\mathrm{I}}}\right)$ as $\ell \nmid D_{K}$. For each integer $n \geq s_{0}$, we put

$$
\Theta_{n}^{\chi}:=\sum_{[a]_{n} \in \mathcal{G}_{n}} F_{\ell}\left(x_{n}(a)\right) \widehat{\chi}\left([a]_{n}\right) \cdot[a]_{n} \in \mathcal{O}\left[\mathcal{G}_{n}\right]
$$

where $\hat{\chi}: \mathcal{G}_{\infty} \rightarrow \mathcal{O}^{\times}$is the $\ell$-adic avatar of $\chi$. One checks by definition that

$$
\left(\varphi_{\pi^{\prime}}^{[m]}\right)^{\dagger}\left(x_{n}(a)\right) \chi(a)=F_{\ell}\left(x_{n}(a)\right) \widehat{\chi}(a), a \in \widehat{K}^{\times},
$$

and hence for each $\nu: \Gamma^{-} \rightarrow \boldsymbol{\mu}_{p^{\infty}}$,

$$
\nu\left(\Theta_{n}^{\chi}\right)=\iota_{\ell} \iota_{p}^{-1}\left(\widehat{\chi} \nu\left(\Theta_{n}^{[m]}\left(f_{\pi^{\prime}}^{\dagger}\right)\right)\right) .
$$

In view of Proposition 4.3 it suffices to show $\nu\left(\Theta_{n}^{\chi}\right) \not \equiv 0(\bmod \lambda)$ for all but finitely many $\nu$.

Let $\mathbf{k}_{\ell}=\mathcal{O} / \varpi \mathcal{O}\left[\boldsymbol{\mu}_{p}\right]$ be the finite extension of the finite field $\mathcal{O} / \varpi \mathcal{O}$ generated by the values of $\boldsymbol{\mu}_{p}$. Put

$$
\mathcal{I}^{\prime}=\left\{g \in \widehat{R}^{\times} \mid g \equiv 1(\bmod \ell), g_{p} \in \mathcal{I}_{p}\right\} .
$$

Then $F_{\ell}(\bmod \lambda) \in \mathcal{M}_{2}\left(\mathcal{I}^{\prime}, \mathbf{k}_{\ell}\right)$. Define $F_{\mathcal{D}_{0}} \in \mathcal{M}_{2}\left(\mathcal{U}_{\mathcal{D}_{0}}^{\prime}, \mathbf{k}_{\ell}\right)$ by

$$
F_{\mathcal{D}_{0}}(g):=\sum_{d \in \mathcal{D}_{0}} \widehat{\chi}(d) F_{\ell}(g d)(\bmod \lambda) \quad\left(\mathcal{U}_{\mathcal{D}_{0}}^{\prime}=\mathcal{I}^{\prime} \cap \mathcal{U}_{\mathcal{D}_{0}}\right) .
$$

It is clear that $F_{\mathcal{D}_{0}}$ is invariant by the Iwahori subgroup $\mathcal{I}_{p}$ and is an $U_{p}$-eigenform with eigenvalue $\alpha_{p}$. Let $p^{s}$ be the order of the Sylow $p$-subgroup of $\mathbf{k}_{\ell}^{\times}$. Let $\nu: \Gamma_{n}^{-} \rightarrow \boldsymbol{\mu}_{p^{\infty}}$ be a character of conductor $p^{n}$ with $n>\max \left\{s, s_{0}\right\}$ (so $\nu: \Gamma_{n}^{-} \hookrightarrow \boldsymbol{\mu}_{p^{\infty}}$ is injective). Put

$$
C_{n}=\left\{\gamma \in \Gamma_{n}^{-} \mid \nu(\gamma) \in \mathbf{k}_{\ell}^{\times}\right\} .
$$

Then we have $C_{n}=\operatorname{Ker}\left(\mathcal{G}_{n} \rightarrow \mathcal{G}_{n-s}\right)$. Let $\mathbf{k}_{\ell}(\nu)$ be the field generated by the values of $\nu$ over $\mathbf{k}_{\ell}$. Since $\mathbf{k}_{\ell}$ contains $\boldsymbol{\mu}_{p}, d_{\nu}:=\left[\mathbf{k}_{\ell}(\nu): \mathbf{k}_{\ell}\right]$ is a $p$-power, and for a $p$-power root of unity $\zeta \in \mathbf{k}_{\ell}(\nu)$, we have

$$
\operatorname{Tr}_{\mathbf{k}_{\ell}(\nu) / \mathbf{k}_{\ell}}(\zeta)= \begin{cases}0 & \cdots \zeta \notin \mathbf{k}_{\ell} \\ d_{\nu} & \cdots \zeta \in \mathbf{k}_{\ell}\end{cases}
$$

It follows from the above that for each $a \in \mathbf{A}_{K, f}^{\times}$,

$$
\begin{aligned}
& \operatorname{Tr}_{\mathbf{k}_{\ell}(\nu) / \mathbf{k}_{\ell}}\left(\alpha_{p}^{n} \widehat{\chi} \nu\left(a^{-1}\right) \cdot \nu\left(\Theta_{n}^{\chi}\right)(\bmod \lambda)\right) \\
= & d_{\nu} \cdot \sum_{[u]_{n} \in C_{n}} \sum_{\tau \in \mathcal{D}_{1}} F_{\mathcal{D}_{0}}\left(x_{n}(a \tau u)\right) \widehat{\chi}(\tau) \\
= & d_{\nu} \cdot \sum_{\tau \in \mathcal{D}_{1}} \sum_{y \in \mathbf{Z} / p^{s} \mathbf{Z}} F_{\mathcal{D}_{0}}\left(x_{n}(a \tau)\left(\begin{array}{cc}
1 & \frac{y}{p^{s}} \\
0 & 1
\end{array}\right)\right) \widehat{\chi}(d) \zeta_{\nu}^{y}
\end{aligned}
$$

for some primitive $p^{s}$-th root of unity $\zeta_{\nu}$. Define $\widetilde{F}_{\mathcal{D}_{0}} \in \mathcal{M}_{2}\left(\mathbf{k}_{\ell}(\nu)\right)$ by

$$
\widetilde{F}_{\mathcal{D}_{0}}(g):=\sum_{y \in \mathbf{Z} / p^{s} \mathbf{Z}} \zeta_{\nu}^{y} \varrho\left(\left(\begin{array}{cc}
1 & \frac{y}{p^{s}} \\
0 & 1
\end{array}\right)\right) F_{\mathcal{D}_{0}}(g) .
$$

Then $\widetilde{F}_{\mathcal{D}_{0}} \in \mathcal{M}_{2}\left(\mathcal{U}^{\prime}, \mathbf{k}_{\ell}(\nu)\right)$ for $\mathcal{U}^{\prime}=\mathcal{I}_{1}\left(p^{2 s}\right) \cap \mathcal{U}_{\mathcal{D}_{0}}^{\prime}$. We can rephrase (5.6) as

$$
\operatorname{Tr}_{\mathbf{k}_{\ell}(\nu) / \mathbf{k}_{\ell}}\left(\alpha_{p}^{n} \widehat{\chi} \nu\left(a^{-1}\right) \cdot \nu\left(\Theta_{n}^{\chi}\right)(\bmod \lambda)\right)=d_{\nu} \cdot \sum_{\tau \in \mathcal{D}_{1}} \widetilde{F}_{\mathcal{D}_{0}}\left(x_{n}(a \tau)\right) \widehat{\chi}(\tau) .
$$


We proceed to show that $\widetilde{F}_{\mathcal{D}_{0}}$ is not Eisenstein. Under our assumptions, $F_{\mathcal{D}_{0}}$ is not Eisenstein by Lemma 5.6 and Lemma [5.8 A simple computation shows that

$$
\begin{aligned}
\sum_{a \in\left(\mathbf{Z} / p^{s} \mathbf{Z}\right)^{\times}} \varrho\left(\left(\begin{array}{cc}
a & 0 \\
0 & 1
\end{array}\right)\right) \widetilde{F}_{\mathcal{D}_{0}} & =\sum_{a \in\left(\mathbf{Z}_{p} / p^{s} \mathbf{Z}\right)^{\times}} \sum_{y \in \mathbf{Z} / p^{s} \mathbf{Z}} \zeta_{\nu}^{a y} \varrho\left(\left(\begin{array}{cc}
1 & \frac{y}{p^{s}} \\
0 & 1
\end{array}\right)\right) F_{\mathcal{D}_{0}} \\
& =p^{s} \cdot F_{\mathcal{D}_{0}}-p^{s-1} \sum_{y \in \mathbf{Z} / p \mathbf{Z}} \varrho\left(\left(\begin{array}{cc}
1 & \frac{y}{p} \\
0 & 1
\end{array}\right)\right) F_{\mathcal{D}_{0}} \\
& =p^{s} \cdot\left(1-p^{-1} \alpha_{p} \cdot \varrho\left(\left(\begin{array}{cc}
p^{-1} & 0 \\
0 & 1
\end{array}\right)\right) F_{\mathcal{D}_{0}} .\right.
\end{aligned}
$$

The above equation implies that $\widetilde{F}_{\mathcal{D}_{0}}$ is not Eisenstein by Lemma 5.5, On the other hand, it is clear that $\mathrm{N}\left(\mathcal{U}^{\prime}\right) \supset \prod_{q \mid D_{K}} \mathbf{Z}_{q}^{\times}$by (5.5). Hence, we can deduce the theorem from Corollary 5.2 in view of (5.7).

\section{THe COMPARISON BETWEen PERIOdS}

In this section, we compare the periods $\Omega_{\pi, N^{-}}$defined in (4.3) and Hida's canonical period $\Omega_{\pi}$. Henceforth, we assume $\ell=p$ and $f_{\pi^{\prime}}^{\dagger}$ is $p$-ordinary. Let $\mathbb{T}\left(\Gamma_{0}(N)\right)$ be the Hecke algebra over $\mathcal{O}$ of the space of elliptic modular forms $S_{k}\left(\Gamma_{0}(N)\right)$. Then $\pi$ gives rise to an $\mathcal{O}$-algebra homomorphism $\lambda_{\pi}: \mathbb{T}\left(\Gamma_{0}(N)\right) \rightarrow \mathcal{O}$ such that $\lambda_{\pi}\left(T_{q}\right)=\operatorname{Tr} \rho_{\pi, p}\left(T_{q}\right)$ for all $q \nmid p N$. Let $\eta_{\pi}(N)$ be an $\mathcal{O}$-generator of the congruence ideal $I_{\pi}(N):=$ $\lambda_{\pi}\left(\operatorname{Ann}_{\mathbb{T}\left(\Gamma_{0}(N)\right)} \operatorname{Ker} \lambda_{\pi}\right) \subset \mathcal{O}$. Then Hida's canonical period $\Omega_{\pi}$ is defined by

$$
\Omega_{\pi}:=\frac{(4 \pi)^{k}\left\|\varphi_{\pi}\right\|_{\Gamma_{0}(N)}}{\eta_{\pi}(N)}
$$

Here we are making use of the fixed embedding $\mathcal{O} \hookrightarrow \mathbf{C}_{p} \simeq$ C. In general, the ratio $\Omega_{\pi, N^{-}} / \Omega_{\pi}$ lies in $\mathcal{O}$. This section is devoted to showing that $\Omega_{\pi, N^{-}} / \Omega_{\pi} \in \mathcal{O}^{\times}$in certain favorable situations. When $k=2$ and $N$ is square-free, under mild assumptions, Pollack and Weston [PW11, Theorem 6.8] even give the formula of the ratio $\Omega_{\pi, N^{-}} / \Omega_{\pi}$ in terms of local Tamagawa components at primes dividing $N^{-}$. They do not need to assume the ordinary hypothesis, but it is not clear to us if their approach is applicable if $k>2$. Nonetheless, it is pointed out in [PW11] that the statement $\Omega_{\pi, N^{-}} / \Omega_{\pi} \in \mathcal{O}^{\times}$is equivalent to the freeness of spaces of modular forms on $B$ over the associated Hecke algebra and the vanishing of these local Tamagawa components. Therefore, it is natural to study the comparison between periods $\Omega_{\pi, N^{-}}$and $\Omega_{\pi}$ by the standard techniques developed by Wiles, Taylor-Wiles, Diamond and Fujiwara in the proof of " $R=T$ " theorems.

Let $G_{\mathbf{Q}}=\operatorname{Gal}(\overline{\mathbf{Q}} / \mathbf{Q})$. For each place $q$, we fix a decomposition of group $G_{q}$ in $G_{\mathbf{Q}}$ and let $I_{q}$ be the inertia group in $G_{q}$. Let $\rho_{0}:=\bar{\rho}_{\pi, p}$ denote the residual Galois representation and let $N_{\rho_{0}}$ be the prime-to- $p$ part of the Artin conductor of $\rho_{0}$. Throughout, we assume the following:

Hypothesis $\left(\mathbf{C R}^{+}\right) . \quad$ (1) The prime $p>k+1$ and $p \nmid N$.

(2) The restriction of $\rho_{0}$ to the absolute Galois group of $\mathbf{Q}\left(\sqrt{(-1)^{\frac{p-1}{2}} p}\right)$ is absolutely irreducible.

(3) If $q \mid N^{-}$and $q \equiv \pm 1(\bmod p)$, then $q \mid N_{\rho_{0}}$.

(4) If $q \| N^{+}$and $q \equiv 1(\bmod p)$, then $q \mid N_{\rho_{0}}$.

(5) $N_{\rho_{0}}$ and $N / N_{\rho_{0}}$ are co-prime.

We will prove the following proposition in $₫ 6.3$ after preparing some notation and recall basic facts in the first two subsections.

Proposition 6.1. Suppose the hypothesis $\left(C R^{+}\right)$holds. If $\rho_{0}$ is ramified at every prime dividing $N^{-}$(i.e. $\left.N^{-} \mid N_{\rho_{0}}\right)$, then the congruence ideal $I_{\pi}(N)$ is generated by $\left\langle f_{\pi^{\prime}}, f_{\pi^{\prime}}\right\rangle_{R}$. In other words, $\Omega_{\pi, N^{-}}=u \cdot \Omega_{\pi}$ for some unit $u \in \mathcal{O}^{\times}$.

6.1. Hecke algebras and congruence ideals. For an open compact subgroup $U \subset \widehat{B}^{\times}$, put $\mathcal{S}(U):=$ $\mathcal{M}_{k}(U, \mathcal{O})$. For $g \in \widehat{B}^{\times}$, Let $\left[U_{1} g U_{2}\right] \in \operatorname{Hom}_{\mathcal{O}}\left(\mathcal{S}\left(U_{2}\right), \mathcal{S}\left(U_{1}\right)\right)$ be the Hecke operator defined by

$$
\left[U_{1} g U_{2}\right] f(g)=\sum_{i} \rho_{k, p}\left(g_{i}\right) f\left(g g_{i}\right) \quad\left(U_{1} g U_{2}=\bigsqcup_{i} g_{i} U_{2}\right) .
$$


Let $M^{+}$be a positive integer with $\left(M^{+}, N^{-}\right)=1$ and let $M=N^{-} M^{+}$. Recall that $R_{M^{+}}$denotes the Eichler order of level $M^{+}$. We put $\mathcal{U}_{M}:=\widehat{R}_{M^{+}}^{\times}$and $\mathcal{S}(M)=\mathcal{S}\left(\mathcal{U}_{M}\right)$. If $q \nmid M$, let $T_{q}$ denote the operator

$$
\left[\mathcal{U}_{M}\left(\begin{array}{ll}
q & 0 \\
0 & 1
\end{array}\right) \mathcal{U}_{M}\right]
$$

and if $q \mid M$, let

$$
U_{q}=\left[\mathcal{U}_{M}\left(\begin{array}{ll}
q & 0 \\
0 & 1
\end{array}\right) \mathcal{U}_{M}\right] \text { for } q \nmid N^{-} \text {and } U_{q}=\left[\mathcal{U}_{M} \varpi_{q} \mathcal{U}_{M}\right] \text { for } q \mid N^{-},
$$

where $\varpi_{q} \in B_{q}^{\times}$such that $\mathrm{N}\left(\varpi_{q}\right)=q$. Let $\mathbb{T}(M)$ be the Hecke algebra generated over $\mathcal{O}$ by Hecke operators $T_{q}$ for $q \nmid M$ and $U_{q}$ for $q \mid M$ in $\operatorname{End}_{\mathcal{O}} \mathcal{S}(M)$. Define the perfect pairing $\langle,\rangle_{M}: \mathcal{S}(M) \times \mathcal{S}(M) \rightarrow \mathcal{O}$ by

$$
\left.\left\langle f_{1}, f_{2}\right\rangle_{M}:=\sum_{[g]}\left\langle f_{1}(g), f_{2}\left(g \tau^{M^{+}}\right)\right\rangle_{k} \cdot\left(\sharp\left(B^{\times} \cap g \mathcal{U}_{M} g^{-1} \widehat{\mathbf{Q}}^{\times}\right) / \mathbf{Q}^{\times}\right)\right)^{-1},
$$

where $[g]$ runs over $B^{\times} \backslash \widehat{B}^{\times} / \mathcal{U}_{M} \widehat{\mathbf{Q}}^{\times}$. It is easy to verify that

$$
\left\langle t f_{1}, f_{2}\right\rangle_{M}=\left\langle f_{1}, t f_{2}\right\rangle_{M} \text { for all } t \in \mathbb{T}(M) .
$$

Let $\lambda_{\pi^{\prime}}: \mathbb{T}(N) \rightarrow \mathcal{O}$ be the $\mathcal{O}$-algebra homomorphism induced by $\pi^{\prime}$. Let $N_{1}^{-}$be the product of prime factors of $N^{-}$but not dividing $N_{\rho_{0}}$. Note that for each $q \mid N_{1}^{-}$we have $q \not \equiv \pm 1(\bmod p)$ and $\operatorname{Tr} \rho_{0}(\operatorname{Frob} q)^{2} \equiv$ $(1+q)^{2}(\bmod p)$ by $\left(\mathrm{CR}^{+}\right)$. Put $N_{\emptyset}:=N_{\rho_{0}} \cdot N_{1}^{-}$. By the level lowering/raising ( $c f$. [Jar99] and [DT94]), there exists a modular lift $\lambda_{\emptyset}: \mathbb{T}\left(N_{\emptyset}\right) \rightarrow \mathcal{O}$ such that $\lambda_{\emptyset}\left(T_{q}\right) \equiv \lambda_{\pi^{\prime}}\left(T_{q}\right)\left(\bmod \mathfrak{m}_{\mathcal{O}}\right)$ for all $q \nmid N\left(\mathfrak{m}_{\mathcal{O}}=\mathcal{O} \cap \lambda\right)$. We write

$$
N=N_{\emptyset} \prod_{q} q^{m_{q}}
$$

Under the hypothesis $\left(\mathrm{CR}^{+}\right)$, it is known ( $c f$. [DT94, pp.435-436]) that

- $m_{q} \leq 2$;

- $m_{q}=0$ unless $q \mid N^{+}$and $q \nmid N_{\emptyset}$;

- If $m_{q}=1$, then $q \not \equiv 1(\bmod p)$.

Let $\Sigma$ be a subset of prime factors of $N / N_{\emptyset}$. Set $N_{\Sigma}:=N_{\emptyset} \cdot \prod_{q \in \Sigma} q^{m_{q}}$. Let $\mathfrak{m}_{\Sigma}$ be the maximal ideal of $\mathbb{T}\left(N_{\Sigma}\right)$ generated by

$$
\mathfrak{m}_{\mathcal{O}}, T_{q}-\lambda_{\emptyset}\left(T_{q}\right) \text { for } q \nmid N_{\Sigma}, U_{q}-\lambda_{\pi^{\prime}}\left(U_{q}\right) \text { for } q \mid N_{\Sigma} .
$$

Let $\mathbb{T}_{\Sigma}:=\mathbb{T}\left(N_{\Sigma}\right)_{\mathfrak{m}_{\Sigma}}$ be the localization at $\mathfrak{m}_{\Sigma}$.

Lemma 6.2. (1) If $q^{2} \mid N_{\Sigma}$, then the Hecke operator $U_{q}=0$ in $\mathbb{T}_{\Sigma}$.

(2) The Hecke algebra $\mathbb{T}_{\Sigma}$ is reduced.

Proof. Part (1) is clear if $q^{2} \mid N_{\emptyset}$, and if $q^{2} \mid N_{\Sigma} / N_{\emptyset}$, it is proved in [Tay06, Corollary 1.8] (cf. [Wil95, Proposition 2.15]). To prove part (2), it suffices to show that $U_{q}$ are semisimple elements in $\mathbb{T}_{\Sigma}$ for $q \mid N_{\Sigma}$. This is clear by part (1) if $q \mid N_{\emptyset}$ or $q^{2} \mid N_{\Sigma}$, and it follows from $\left(\mathrm{CR}^{+}\right)$(4) if $q \| N_{\Sigma} / N_{\emptyset}$.

Let $\varepsilon_{p}: G_{\mathbf{Q}} \rightarrow \mathbf{Z}_{p}^{\times}$be the $p$-adic cyclotomic character. It is well known that there exists a Galois representation

such that

$$
\rho_{\Sigma}: G_{\mathbf{Q}} \rightarrow \mathrm{GL}_{2}\left(\mathbb{T}_{\Sigma}\right)
$$

- $\rho_{\Sigma}$ is unramified outside $p N_{\Sigma}$.

- $\operatorname{Tr} \rho_{\Sigma}\left(\operatorname{Frob}_{q}\right)=T_{q}$ for all $q \nmid p N_{\Sigma}$ and $\operatorname{det} \rho_{\Sigma}=\varepsilon_{p}$.

- There exists a character $\delta_{p}: G_{p} \rightarrow \mathbb{T}_{\Sigma}^{\times}$such that

$$
\left.\rho_{\Sigma}\right|_{G_{p}} \sim\left(\begin{array}{cc}
\delta_{p}^{-1} \varepsilon_{p} & * \\
0 & \delta_{p}
\end{array}\right) \text { and }\left.\delta_{p}\right|_{I_{p}}=\varepsilon^{(2-k) / 2}
$$

- For each $q \| N_{\Sigma} / N_{1}^{-}$, there exists a character $\delta_{\Sigma, q}: G_{q} \rightarrow \mathbb{T}_{\Sigma}^{\times}$such that

$$
\left.\rho_{\Sigma}\right|_{G_{q}} \sim\left(\begin{array}{cc}
\delta_{\Sigma, q}^{-1} \varepsilon_{p} & * \\
0 & \delta_{\Sigma, q}
\end{array}\right) \text { and } \delta_{\Sigma, q}\left(\operatorname{Frob}_{q}\right)=U_{q}
$$

Here $\mathrm{Frob}_{q}$ is the arithmetic Frobenius at $q$. 
- For $q \mid N_{1}^{-}$,

$$
\left.\rho_{\Sigma}\right|_{G_{q}} \sim\left(\begin{array}{cc} 
\pm \varepsilon_{p} & * \\
0 & \pm 1
\end{array}\right), * \in \mathfrak{m}_{\Sigma}
$$

Let $q \mid N / N_{\emptyset}$ such that $q \notin \Sigma$. We define an element $u_{\Sigma, q} \in \mathbb{T}_{\Sigma}$ and a level-raising map $L_{q}: \mathcal{S}_{\Sigma} \rightarrow \mathcal{S}_{\Sigma \cup\{q\}}$ as follows. If $m_{q}=2$, set $u_{\Sigma, q}:=0$ and define

$$
L_{q}(f)=q f-\left(\begin{array}{ll}
1 & 0 \\
0 & q
\end{array}\right) T_{q} f+\left(\begin{array}{cc}
1 & 0 \\
0 & q^{2}
\end{array}\right) f .
$$

If $m_{q}=1$, then the Hecke polynomial $P_{q}(X)=X^{2}-T_{q} X+q \in \mathbb{T}_{\Sigma}[X]$ is congruent to $\left(X-\epsilon_{q} q\right)\left(X-\epsilon_{q}\right)$ modulo $\mathfrak{m}_{\Sigma}$ for some $\epsilon_{q} \in\{ \pm 1\}$. Since $q \not \equiv 1(\bmod p)$, there exists a unique root $u_{\Sigma, q} \in \mathbb{T}_{\Sigma}$ of $P_{q}(X)$ such that $u_{\Sigma, q} \equiv \epsilon_{q}\left(\bmod \mathfrak{m}_{\Sigma}\right)$ by Hensel's lemma. Define

$$
L_{q}(f)=u_{\Sigma, q} f-\left(\begin{array}{ll}
1 & 0 \\
0 & q
\end{array}\right) f
$$

In either case, it is easy to verify that $U_{q} \circ L_{q}=L_{q} \circ u_{\Sigma, q}$. Moreover, $L_{q}$ induces a surjective map $\mathbb{T}_{\Sigma \cup\{q\}} \rightarrow \mathbb{T}_{\Sigma}$, sending $U_{q}$ to $u_{\Sigma, q}$ by the following lemma:

Lemma 6.3. The map $L_{q}$ is injective, and $\mathcal{S}_{\Sigma \cup\{q\}} / L_{q}\left(\mathcal{S}_{\Sigma}\right)$ is a free $\mathcal{O}$-module.

Proof. This is [Tay06, Lemma 3.1] (cf. Lemma 5.5).

The above construction gives rise to a homomorphism $\mathbb{T}_{\Sigma} \rightarrow \mathbb{T}_{\emptyset}$. If $\lambda: \mathbb{T}_{\Sigma} \rightarrow \mathcal{O}$ is an $\mathcal{O}$-algebra homomorphism, we write $I_{\lambda}$ for the kernel of $\lambda$ and put

$$
\mathcal{S}_{\Sigma}[\lambda]:=\left\{x \in \mathcal{S}_{\Sigma} \mid I_{\lambda} x=0\right\}
$$

Let $\lambda_{\Sigma}: \mathbb{T}_{\Sigma} \rightarrow \mathbb{T}_{\emptyset} \stackrel{\lambda_{\emptyset}}{\longrightarrow} \mathcal{O}$ be the composition. The $\mathcal{O}$-module $\mathcal{S}_{\Sigma}\left[\lambda_{\Sigma}\right]$ is free of rank one by the strong multiplicity one theorem and the reducedness of $\mathbb{T}_{\Sigma}$. Let $\mathcal{S}_{\Sigma}\left[\lambda_{\Sigma}\right]^{\perp}$ be the $\mathcal{O}$-module defined by

$$
\mathcal{S}_{\Sigma}\left[\lambda_{\Sigma}\right]^{\perp}=\left\{x \in \mathcal{S}_{\Sigma}\left[\lambda_{\Sigma}\right] \otimes_{\mathcal{O}} E \mid\langle x, y\rangle_{N_{\Sigma}} \in \mathcal{O} \text { for all } y \in \mathcal{S}_{\Sigma}\left[\lambda_{\Sigma}\right]\right\}
$$

where $E$ is the fraction field of $\mathcal{O}$. Then $\mathcal{S}_{\Sigma}\left[\lambda_{\Sigma}\right]^{\perp} \supset \mathcal{S}_{\Sigma}\left[\lambda_{\Sigma}\right]$. We let $C\left(N_{\Sigma}\right):=\mathcal{S}_{\Sigma}\left[\lambda_{\Sigma}\right]^{\perp} / \mathcal{S}_{\Sigma}\left[\lambda_{\Sigma}\right]$ be the congruence module of $\lambda_{\Sigma}$ and let $\eta_{\Sigma}=\lambda_{\Sigma}\left(\operatorname{Ann}_{\mathbb{T}_{\Sigma}} I_{\lambda_{\Sigma}}\right)$ be the congruence ideal of $\lambda_{\Sigma}$. It is known that

$$
\sharp C\left(N_{\Sigma}\right) \leq \sharp\left(\mathcal{O} / \eta_{\Sigma}\right)
$$

and the equality holds if $\mathcal{S}_{\Sigma}$ is free over $\mathbb{T}_{\Sigma}$ (so $\mathbb{T}_{\Sigma}$ is Gorenstein).

Lemma 6.4. If $q \| N / N_{\emptyset}$ and $q \notin \Sigma$, then we have

$$
\sharp C\left(N_{\Sigma \cup\{q\}}\right)=\sharp C\left(N_{\Sigma}\right) \cdot \sharp\left(\mathcal{O} /\left(\lambda_{\emptyset}\left(u_{\emptyset, q}\right)^{2}-1\right) \mathcal{O}\right) .
$$

Proof. Note that $\lambda_{\emptyset}\left(u_{\emptyset, q}\right) \neq 1$ as $\lambda_{\emptyset}$ is unramified outside $N_{\emptyset}$. Let $L_{q}^{*}: \mathcal{S}_{\Sigma} \rightarrow \mathcal{S}_{\Sigma \cup\{q\}}$ be the adjoint map of $L_{q}$ with respect to $\langle,\rangle_{N_{\Sigma}}$ and $\langle,\rangle_{N_{\Sigma \cup\{q\}}}$. It follows from Lemma 6.3 that

$$
L_{q}\left(\mathcal{S}_{\Sigma}\left[\lambda_{\Sigma}\right]\right)=\mathcal{S}_{\Sigma \cup\{q\}}\left[\lambda_{\Sigma \cup\{q\}}\right] ; L_{q}^{*}\left(\mathcal{S}_{\Sigma \cup\{q\}}\left[\lambda_{\Sigma \cup\{q\}}\right]^{\perp}\right)=\mathcal{S}_{\Sigma}\left[\lambda_{\Sigma}\right]^{\perp} .
$$

Let $\mathcal{U}=\mathcal{U}_{N_{\Sigma}}$ and $\mathcal{U}_{1}=\mathcal{U}_{N_{\Sigma \cup\{q\}}}$. A direct computation shows that

Therefore, we find that

$$
L_{q}^{*}=u_{\Sigma, q} \cdot\left[\mathcal{U}\left(\begin{array}{ll}
q & 0 \\
0 & 1
\end{array}\right) \mathcal{U}_{1}\right]-\left[\mathcal{U U}_{1}\right]
$$

$$
\begin{aligned}
L_{q}^{*} \circ L_{q} & =u_{\Sigma, q} \cdot\left[\mathcal{U}\left(\begin{array}{ll}
q & 0 \\
0 & 1
\end{array}\right) \mathcal{U}\right] \cdot u_{\Sigma, q}-u_{\Sigma, q} \cdot\left[\mathcal{U}\left(\begin{array}{ll}
q & 0 \\
0 & q
\end{array}\right) \mathcal{U}\right] \cdot(1+q)-[\mathcal{U} \mathcal{U}] \cdot(1+q) u_{\Sigma, q}+\left[\mathcal{U}\left(\begin{array}{ll}
1 & 0 \\
0 & q
\end{array}\right) \mathcal{U}\right] \\
& =\left(1+u_{\Sigma, q}^{2}\right) \cdot T_{q}-2 u_{\Sigma, q} \cdot(1+q) \\
& =u_{\Sigma, q}^{-1}\left(u_{\Sigma, q}^{2}-1\right)\left(u_{\Sigma, q}^{2}-q\right) .
\end{aligned}
$$

Since $u_{\Sigma, q}^{2}-q \equiv 1-q \not \equiv 0\left(\bmod \mathfrak{m}_{\Sigma}\right)$, by (‥4) we find that

$$
\begin{aligned}
\sharp C\left(N_{\Sigma \cup\{q\}}\right) & =\sharp\left(\mathcal{S}_{\Sigma}\left[\lambda_{\Sigma}\right]^{\perp} / L_{q}^{*} \circ L_{q}\left(\mathcal{S}_{\Sigma}\left[\lambda_{\Sigma}\right]\right)\right) \\
& \left.=\sharp C\left(N_{\Sigma}\right)\right) \cdot \sharp \mathcal{O} /\left(\lambda_{\emptyset}\left(u_{\emptyset, q}\right)^{2}-1\right) \mathcal{O} .
\end{aligned}
$$


6.2. Deformation rings and Selmer groups. We introduce a certain deformation ring. Recall that $\rho$ is a deformation of $\rho_{0}$ if $\rho\left(\bmod \mathfrak{m}_{A}\right) \simeq \rho_{0}$. Consider the functor $\mathfrak{D}_{\Sigma}$ from local Noetherian complete $\mathcal{O}$-algebras with the residual field $\mathbf{k}$ to sets which sends $A$ with the maximal ideal $\mathfrak{m}_{A}$ to the isomorphism classes of deformations $\rho: G_{\mathbf{Q}} \rightarrow \mathrm{GL}_{2}(A)$ of $\rho_{0}$ satisfying:

(D1) $\operatorname{det} \rho=\varepsilon_{p}$;

(D2) $\rho$ is minimally ramified outside $N_{1}^{-} \Sigma$ in the sense of [Dia97a, Definition 3.1];

(D3) There exists a character $\delta_{p}: G_{q} \rightarrow A^{\times}$such that

$$
\left.\rho\right|_{G_{p}} \sim\left(\begin{array}{cc}
\delta_{p}^{-1} \varepsilon_{p} & * \\
0 & \delta_{p}
\end{array}\right) \text { and }\left.\delta_{p}\right|_{I_{p}}=\varepsilon_{p}^{(2-k) / 2}
$$

(D4) For each $q \| N_{\Sigma} / N_{\emptyset}$, there exists a unramified character $\delta_{q}: G_{q} \rightarrow A^{\times}$such that

$$
\left.\rho\right|_{G_{q}} \sim\left(\begin{array}{cc}
\delta_{q}^{-1} \varepsilon_{p} & * \\
0 & \delta_{q}
\end{array}\right) \text { and } \delta_{q}\left(\operatorname{Frob}_{q}\right) \equiv 1\left(\bmod \mathfrak{m}_{A}\right) .
$$

(D5) If $q \mid N_{1}^{-}$, then $\left.\rho\right|_{G_{q}}$ satisfies the sp-condition in [Ter03, Definition 2.2]. Namely,

$$
\left.\rho\right|_{G_{q}} \sim\left(\begin{array}{cc} 
\pm \varepsilon_{p} & * \\
0 & \pm 1
\end{array}\right), * \in \mathfrak{m}_{A} .
$$

Under $\left(\mathrm{CR}^{+}\right)$, it is a standard fact that $\mathfrak{D}_{\Sigma}$ is represented by the universal deformation

$$
\rho_{R_{\Sigma}}: G_{\mathbf{Q}} \rightarrow \mathrm{GL}_{2}\left(R_{\Sigma}\right) .
$$

The universal property of $R_{\Sigma}$ gives rise to $\mathcal{O}$-algebra homomorphisms $R_{\Sigma} \rightarrow R_{\emptyset}$ and $R_{\Sigma} \rightarrow \mathbb{T}_{\Sigma}$ under which $\rho_{R_{\Sigma}}$ pushes forward to $\rho_{R_{\emptyset}}$ and $\rho_{\Sigma}$ respectively.

Lemma 6.5. The map $R_{\Sigma} \rightarrow \mathbb{T}_{\Sigma}$ is a surjection.

Proof. By Lemma 6.2 $\mathbb{T}_{\Sigma}$ is generated by $T_{q}$ for $q \nmid N_{\Sigma}$ and $U_{q}$ for $q \| N_{\Sigma}$. If $q \nmid N_{\Sigma}$, we have $\operatorname{Tr} \rho_{R_{\Sigma}}\left(\operatorname{Frob}_{q}\right) \mapsto T_{q}$. If $q \mid N_{1}^{-}$, then $U_{q}= \pm 1$ by the sp-condition. If $q \| N_{\Sigma} / N_{1}^{-}$, then

$$
\left.\rho_{R_{\Sigma}}\right|_{G_{q}} \simeq\left(\begin{array}{cc}
\delta_{R_{\Sigma}, q}^{-1} \varepsilon_{p} & * \\
& \delta_{R_{\Sigma}, q}
\end{array}\right) \text { for a unramified character } \delta_{R_{\Sigma}, q}: G_{q} \rightarrow R_{\Sigma}^{\times}
$$

such that $\delta_{R_{\Sigma}, q}\left(\operatorname{Frob}_{q}\right) \equiv 1\left(\bmod \mathfrak{m}_{R_{\Sigma}}\right)$, and we have $\delta_{R_{\Sigma}, q}\left(\operatorname{Frob}_{q}\right) \mapsto U_{q}$.

Let $\wp_{\Sigma}$ be the kernel of the $\mathcal{O}$-algebra morphism:

$$
R_{\Sigma} \rightarrow R_{\emptyset} \rightarrow \mathbb{T}_{\emptyset} \stackrel{\lambda_{\emptyset}}{\longrightarrow} \mathcal{O} .
$$

Let $W_{\rho}$ denote the discrete Galois module $\operatorname{ad}^{0} \rho_{\lambda_{\emptyset}} \otimes E / \mathcal{O}$. Define the subspace $W_{\rho}^{+}$by

$$
W_{\rho}^{+}=\left\{\left(\begin{array}{cc}
a & b \\
0 & -a
\end{array}\right) \mid a, b \in E / \mathcal{O}\right\} \subset W_{\rho}=\left\{\left(\begin{array}{cc}
a & b \\
c & -a
\end{array}\right) \mid a, b, c \in E / \mathcal{O}\right\} .
$$

We define the subgroup $L_{q} \subset H^{1}\left(\mathbf{Q}_{q}, W_{\rho}\right)$ as follows. We denote $L_{q}=H^{1}\left(\mathbf{Q}_{q}, W_{\rho}^{+}\right)$if $q \| N_{\Sigma} / N_{\emptyset}, L_{q}=$ $\operatorname{ker}\left\{H^{1}\left(\mathbf{Q}_{q}, W_{\rho}\right) \rightarrow H^{1}\left(\langle F\rangle, W_{\rho}\right)\right\}$, where $F$ is a lifting of Frob $_{q}$ in $G_{q}$ if $q \mid N_{1}^{-}$, and $L_{q}=H_{f}^{1}\left(\mathbf{Q}_{q}, W_{\rho}\right)$ be the local Bloch-Kato group otherwise. Define the Selmer group by

$$
\operatorname{Sel}_{\Sigma}\left(W_{\rho}\right):=\operatorname{ker}\left\{H^{1}\left(\mathbf{Q}, W_{\rho}\right) \rightarrow \prod_{q} \frac{H^{1}\left(\mathbf{Q}_{q}, W_{\rho}\right)}{L_{q}}\right\} .
$$

It is not difficult to show that we have an $\mathcal{O}$-module isomorphism:

$$
\operatorname{Hom}_{\mathcal{O}}\left(\wp_{\Sigma} / \wp_{\Sigma}^{2}, E / \mathcal{O}\right) \simeq \operatorname{Sel}_{\Sigma}\left(W_{\rho}\right) .
$$

Let $Q_{2}$ be the set of prime factors $q \mid N / N_{\emptyset}$ with $m_{q}=2$. 
Lemma 6.6. We have a natural inclusion map

$$
H_{f}^{1}\left(\mathbf{Q}_{q}, W_{\rho}\right) \hookrightarrow H^{1}\left(\mathbf{Q}_{q}, W_{\rho}^{+}\right) .
$$

In particular, if $\Sigma \supset Q_{2}$, then we have

$$
\sharp\left(\operatorname{Sel}_{\Sigma}\left(W_{\rho}\right)\right) / \sharp\left(\operatorname{Sel}_{Q_{2}}\left(W_{\rho}\right)\right) \mid \prod_{q \| N_{\Sigma} / N_{\emptyset}} \sharp\left(H^{1}\left(I_{q}, W_{\rho}^{+}\right)^{G_{q}}\right) .
$$

Proof. Let $q \| N / N_{\emptyset}$. Let $W_{\rho}^{-}:=W_{\rho} / W_{\rho}^{+}$be a discrete $G_{q}$-module of $\mathcal{O}$-corank one. Consider the following diagram:

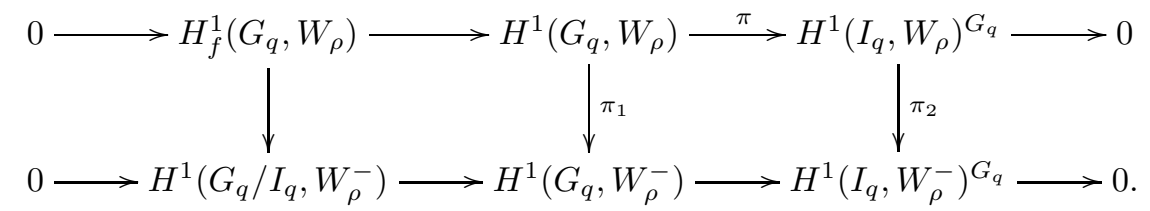

Since $q \not \equiv 1(\bmod p), H^{0}\left(G_{q}, W_{\rho}^{-}\right)=H^{1}\left(G_{q} / I_{q}, W_{\rho}^{-}\right)=0$. It follows that $\operatorname{ker} \pi_{1}=L_{q}=H^{1}\left(G_{q}, W_{\rho}^{+}\right)$and $H^{1}\left(G_{q} / I_{q}, W_{\rho}^{+}\right) \simeq H_{f}^{1}\left(G_{q}, W_{\rho}\right)$. By the snake lemma, $H_{f}^{1}\left(G_{q}, W_{\rho}\right)$ is a submodule of $L_{q}$. The second assertion follows from the exact sequence

$$
0 \rightarrow \operatorname{Sel}_{Q_{2}}\left(W_{\rho}\right) \rightarrow \operatorname{Sel}_{\Sigma}\left(W_{\rho}\right) \rightarrow \prod_{q \| N_{\Sigma} / N_{\emptyset}} \frac{L_{q}}{H_{f}^{1}\left(G_{q}, W_{\rho}\right)}
$$

and the isomorphism

$$
H^{1}\left(I_{q}, W_{\rho}^{+}\right)^{G_{q}} \simeq H^{1}\left(G_{q}, W_{\rho}^{+}\right) / H^{1}\left(G_{q} / I_{q}, W_{\rho}^{+}\right) \simeq L_{q} / H_{f}^{1}\left(G_{q}, W_{\rho}\right) .
$$

Corollary 6.7. If $\Sigma$ is the set of prime factors of $N / N_{\emptyset}$, then we have

$$
\sharp\left(\wp_{\Sigma} / \wp_{\Sigma}^{2}\right) \mid \sharp\left(\wp_{Q_{2}} / \wp_{Q_{2}}^{2}\right) \cdot \prod_{q \| N / N_{\emptyset}} \sharp\left(\mathcal{O} /\left(\lambda_{\emptyset}\left(u_{\emptyset, q}\right)^{2}-1\right) \mathcal{O}\right) .
$$

Proof. By (6.5) and Lemma 6.6.

$$
\sharp \operatorname{ker}\left(\wp_{\Sigma} / \wp_{\Sigma}^{2} \rightarrow \wp_{Q_{2}} / \wp_{Q_{2}}^{2}\right)=\sharp\left(\operatorname{Sel}_{\Sigma}\left(W_{\rho}\right) / \operatorname{Sel}_{Q_{2}}\left(W_{\rho}\right)\right)
$$

divides

$$
\begin{aligned}
\prod_{q \| N / N_{\emptyset}} \sharp\left(H^{1}\left(I_{q}, W_{\rho}^{+}\right)^{G_{q}}\right) & =\prod_{q \| N / N_{\emptyset}} \sharp H^{0}\left(G_{q}, W_{\rho}^{+}(-1)\right) \\
& =\prod_{q \| N / N_{\emptyset}} \sharp\left(\mathcal{O} /\left((q-1)\left(\lambda_{\emptyset}\left(u_{\emptyset, q}\right)^{2}-1\right) \mathcal{O}\right)\right) .
\end{aligned}
$$

6.3. Proof of Proposition 6.1. Let $\Sigma$ be the set of prime factors of $N / N_{\emptyset}$. We begin with the following proposition on the freeness of the Hecke module $\mathcal{S}_{\Sigma}$.

Proposition 6.8. With the hypothesis $\left(\mathrm{CR}^{+}\right), \mathcal{S}_{\Sigma}$ is a free $\mathbb{T}_{\Sigma}$-module of rank one.

Proof. First consider the case $\Sigma=\emptyset$. We have

$$
\operatorname{Hom}\left(\mathfrak{m}_{\emptyset} / \mathfrak{m}_{\emptyset}^{2}, \mathcal{O} / \mathfrak{m}_{\mathcal{O}}\right)=\operatorname{ker}\left\{H^{1}\left(\mathbf{Q}, \operatorname{ad}^{0} \rho_{0}\right) \rightarrow \prod_{q} \frac{H^{1}\left(\mathbf{Q}_{q}, \operatorname{ad}^{0} \rho_{0}\right)}{\mathcal{L}_{q}}\right\},
$$

where $\mathcal{L}_{q}=H_{f}^{1}\left(\mathbf{Q}_{q}, \operatorname{ad}^{0} \rho_{0}\right)$ if $q \nmid N_{1}^{-}$and $\mathcal{L}_{q}=\operatorname{ker}\left\{H^{1}\left(\mathbf{Q}_{q}, \operatorname{ad}^{0} \rho_{0}\right) \rightarrow H^{1}\left(\langle F\rangle, \operatorname{ad}^{0} \rho_{0}\right)\right\}$ for a lifting $F$ of Frob $_{q}$ in $G_{q}$ if $q \mid N_{1}^{-}$. This is the minimal case in the sense that $\sharp\left(\mathcal{L}_{q}\right)=\sharp\left(H^{0}\left(G_{q}, \operatorname{ad}^{0} \rho_{0}\right)\right)$ for all $q$ (Ter03. $\S 3.4])$. Using the Taylor-Wiles system constructed in [Tay06, §2], we deduce that $\mathcal{S}_{\emptyset}$ is a free $\mathbb{T}_{\emptyset}$-module of rank one and

$$
\sharp\left(\wp_{\emptyset} / \wp_{\emptyset}^{2}\right)=\sharp C\left(N_{\emptyset}\right)=\sharp\left(\mathcal{O} / \eta_{\emptyset}\right) .
$$

Furthermore, the argument in [Tay06, §3] shows that

$$
\sharp\left(\wp Q_{2} / \wp_{Q_{2}}^{2}\right)=\sharp C\left(N_{Q_{2}}\right)=\sharp\left(\mathcal{O} / \eta_{Q_{2}}\right) .
$$


Combined with Lemma 6.4 and Corollary 6.7, the above equation yields that

$$
\sharp\left(\wp_{\Sigma} / \wp_{\Sigma}^{2}\right)|\sharp C(N)| \sharp\left(\mathcal{O} / \eta_{\Sigma}\right) .
$$

The proposition follows from [Dia97b, Theorem 2.4].

Now we are ready to prove Proposition 6.1. The Jacquet-Langlands correspondence induces a surjective $\mathcal{O}$-algebra homomorphism $J L^{*}: \mathbb{T}\left(\Gamma_{0}(N)\right)_{\mathfrak{m}} \rightarrow \mathbb{T}_{\Sigma}$ such that $\lambda_{\pi}=J L^{*} \circ \lambda_{\pi^{\prime}}$, where $\mathfrak{m}$ is the maximal ideal containing ker $\lambda_{\pi}$. The assumption $N^{-} \mid N_{\rho_{0}}$ implies $J L^{*}$ is an isomorphism. On the other hand, by definition $\mathcal{S}_{\Sigma}\left[\lambda_{\pi^{\prime}}\right]=\mathcal{O} \cdot \widehat{f}_{\pi^{\prime}}$ and $\left\langle f_{\pi^{\prime}}, f_{\pi^{\prime}}\right\rangle_{R}=\left\langle\widehat{f}_{\pi^{\prime}}, \widehat{f}_{\pi^{\prime}}\right\rangle_{N_{B}}$. Therefore, by Proposition 6.8 we conclude that

$$
\sharp\left(\mathcal{O} / I_{\pi}(N)\right)=\sharp\left(\mathcal{S}_{\Sigma}\left[\lambda_{\pi^{\prime}}\right]^{\perp} / \mathcal{S}_{\Sigma}\left[\lambda_{\pi^{\prime}}\right]\right)=\sharp\left(\mathcal{O} /\left\langle f_{\pi^{\prime}}, f_{\pi^{\prime}}\right\rangle_{R} \mathcal{O}\right) .
$$

This completes the proof.

\section{REFERENCES}

[BD96] M. Bertolini and H. Darmon, Heegner points on Mumford-Tate curves, Invent. Math. 126 (1996), no. 3, 413-456.

[BD05] Iwasawa's main conjecture for elliptic curves over anticyclotomic $\mathbb{Z}_{p}$-extensions, Ann. of Math. (2) 162 (2005), no. $1,1-64$.

[BDIS02] Massimo Bertolini, Henri Darmon, Adrian Iovita, and Michael Spiess, Teitelbaum's exceptional zero conjecture in the anticyclotomic setting, Amer. J. Math. 124 (2002), no. 2, 411-449.

[Cas73] W. Casselman, On some results of Atkin and Lehner, Math. Ann. 201 (1973), 301-314.

[CV05] C. Cornut and V. Vatsal, CM points and quaternion algebras, Doc. Math. 10 (2005), 263-309.

[Dia97a] F. Diamond, An extension of Wiles' results, Modular forms and Fermat's last theorem (Boston, MA, 1995), Springer, New York, 1997, pp. 475-489.

[Dia97b] - The Taylor-Wiles construction and multiplicity one, Invent. Math. 128 (1997), no. 2, 379-391.

[DT94] F. Diamond and R. Taylor, Nonoptimal levels of mod l modular representations, Invent. Math. 115 (1994), no. 3, 435-462.

[Hid81] H. Hida, Congruence of cusp forms and special values of their zeta functions, Invent. Math. 63 (1981), no. 2, 225-261.

[Jac72] H. Jacquet, Automorphic forms on GL(2). Part II, Lecture Notes in Mathematics, Vol. 278, Springer-Verlag, Berlin, 1972.

[Jar99] Frazer Jarvis, Level lowering for modular mod l representations over totally real fields, Math. Ann. 313 (1999), no. 1, $141-160$.

[JL70] H. Jacquet and R. P. Langlands, Automorphic forms on GL(2), Lecture Notes in Mathematics, Vol. 114, SpringerVerlag, Berlin, 1970.

[LV10] M. Longo and S. Vigni, On the vanishing of Selmer groups for elliptic curves over ring class fields, J. Number Theory 130 (2010), no. 1, 128-163.

[PTB11] D. Prasad and R. Takloo-Bighash, Bessel models for GSp(4), J. Reine Angew. Math. 655 (2011), $189-243$.

[PW11] R. Pollack and T. Weston, On anticyclotomic $\mu$-invariants of modular forms, Compos. Math. 147 (2011), no. 5, 1353-1381.

[Sch02] Ralf Schmidt, Some remarks on local newforms for GL(2), J. Ramanujan Math. Soc. 17 (2002), no. 2, $115-147$. MR 1913897 (2003g:11056)

[Sin87] W. Sinnott, On a theorem of L. Washington, Astérisque (1987), no. 147-148, 209-224, 344, Journées arithmétiques de Besançon (Besançon, 1985).

[SU10] C. Skinner and E. Urban, The Iwasawa main conjectures for $\mathrm{GL}_{2}$, Preprint, 226 p., November, 2010.

[Tay06] R. Taylor, On the meromorphic continuation of degree two L-functions, Doc. Math. (2006), no. Extra Vol., 729-779 (electronic).

[Ter03] L. Terracini, A Taylor-Wiles system for quaternionic Hecke algebras, Compositio Math. 137 (2003), no. 1, $23-47$.

[Vat02] V. Vatsal, Uniform distribution of Heegner points, Invent. Math. 148 (2002), no. 1, 1-46.

[Vat03] Special values of anticyclotomic L-functions, Duke Math. J. 116 (2003), no. 2, 219-261.

[VO12] Jeanine Van Order, On the quaternionic p-adic L-functions associated to Hilbert modular eigenforms, Int. J. Number Theory 8 (2012), no. 4, 1005-1039.

[Wal85] J.-L. Waldspurger, Sur les valeurs de certaines fonctions L automorphes en leur centre de symétrie, Compositio Math. 54 (1985), no. 2, 173-242.

[Wil95] A. Wiles, Modular elliptic curves and Fermat's last theorem, Ann. of Math. (2) 141 (1995), no. 3, $443-551$.

[Yua05] H. Yuan, Special Vaule Formulae of Rankin-Selberg L-functions, Ph.D. thesis, University of Pennsylvania, 2005.

[Zha04] Shou-Wu Zhang, Gross-Zagier formula for GL(2). II, Heegner points and Rankin L-series, Math. Sci. Res. Inst. Publ., vol. 49, Cambridge Univ. Press, Cambridge, 2004, pp. 191-214. MR 2083213 (2005k:11121)

Department of Mathematics, Graduate School of Science, Kyoto University, Kitashirakawa-Oiwakecho, SakyoKU, Куото, 606-8502, JaPAN. 
The Hakubi Center for Advanced Research, Kyoto University, Yoshida-Ushinomiya-cho, Sakyo-ku, Kyoto, 606-8302, JAPAN

E-mail address: chida@math.kyoto-u.ac.jp

Department of Mathematics, National Taiman University, No. 1, Sec. 4, Roosevelt Road, Taipei 10617, Taiwan E-mail address: mlhsieh@math.ntu.edu.tw 